\title{
The Loudspeaker: \\ Transformative and Tactile Aspects of the Object
}

By

\section{Jason Wright}

Student ID: 301024974

\author{
A thesis submitted to Massey University and \\ Victoria University of Wellington in fulfillment of \\ the requirements for the degree of \\ Master of Music in Composition
}

New Zealand School of Music 


\begin{abstract}
Traditionally the loudspeaker has been viewed as a functional object. Whilst also serving as a design artifact and object of consumer fetishism, it is subject to the overriding purpose of the reproduction and replication of sound material. However, within sound-based arts, other understandings and uses of the loudspeaker are emerging. This object is now being recognised for its ability to transform sound, taking a proactive stance within sound-based arts. Through exploration of the psychoacoustic effects of the object, the loudspeaker's ability to transform the musical object is being recognised. At the opposing pole, through physical interaction and exploiting physical aspects of the object, the loudspeaker becomes instrumental in creating its own musical objects.
\end{abstract}

My research delves into examples of sound and sonic art where the loudspeaker is exploited for its aural, physical and visual characteristics; where specific qualities of loudspeakers, as well as various transformations of the loudspeaker's physical construction and function, are integral to a particular work. Whilst examining sound installation, sculpture and performance, I will also be unpacking the loudspeaker as an object that permeates everyday life, not least within a consumerist context, and how we have come to understand and listen to loudspeakers, looking at the effects this may have on our perception of sound and listening more generally. 


\section{Acknowledgements}

First and foremost my thanks go to my supervisor, Dr. Dugal McKinnon, whose expertise in this field has been invaluable in realising this research. His ability to constantly point me in right direction has been indispensable and his teaching and feedback has helped to shape both my research and my Sonic Arts practice.

To those who have helped realise my portfolio of works for this project, a huge thanks. I would not have been able to complete this work without these people: Chris Black, Boofa, Bridget Johnson, Jim Murphy and Jason Post. The input and feedback I received from you for these projects was indispensable. The quality of the performances, video, photography and sound recording is amazing. You are all amazing artists that I am privileged to know and work with.

Thanks to Casimar Larkin for the amazing job on proof reading. Your expertise in grammar and punctuation was much needed. Also thanks for helping me learn words! Again, thanks to Bridget 'funk-coding' Johnson for encouraging me to stay at school and work towards my Masters. Your friendship and encouragement over the last year and a half (and the previous four years!) was a huge part in completing this work. You're an amazing person and artist and I'm looking forward to seeing all the amazing work you're going to do in the future!! 
Finally, to my Mum, Caroline Rochecouste, and my Aunty, Veronique Rochecouste: the love, support and encouragement I received from you over the course of this work enabled me to finish. I couldn't have done it without you. Thankyou, I love you both lots. 


\section{Table of Contents}

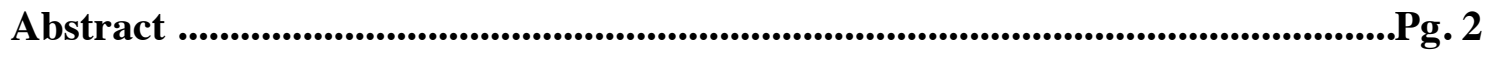

Acknowledgements .............................................................................................................Pg. 3

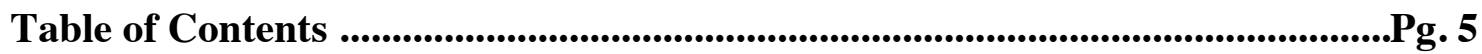

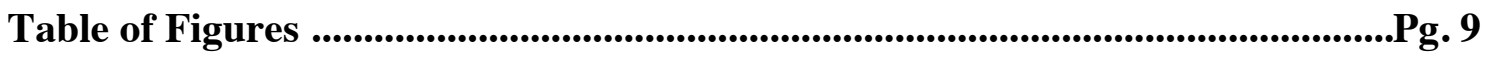

Section A: Exegesis

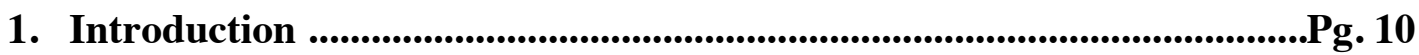

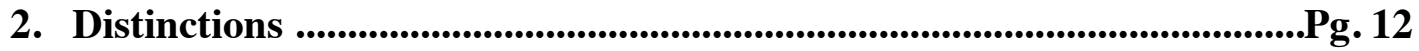

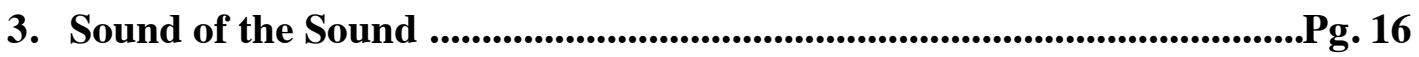

4. Transformation of the Musical Object .......................................................Pg. 19

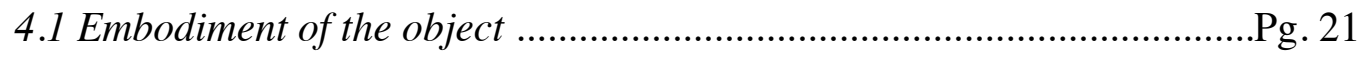

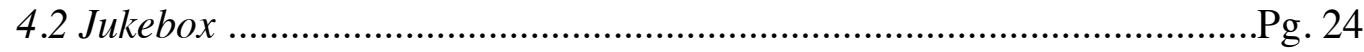

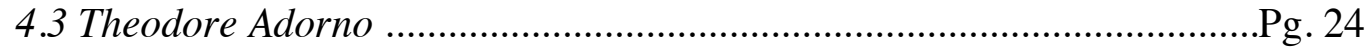

4.4 Leopold Stokowski ............................................................................ 26

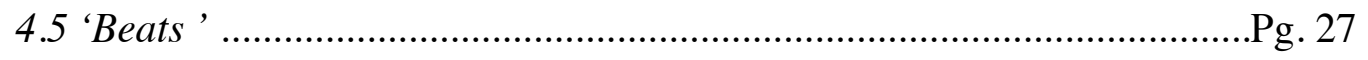

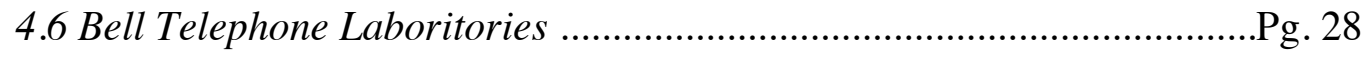

4.7 The Dancehall Sound-System ............................................................Pg. 30

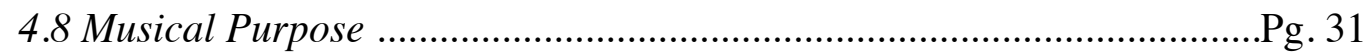

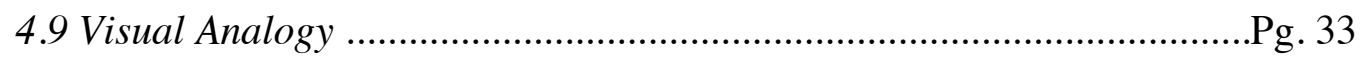

5. The Loudspeaker as Instrument ............................................................Pg. 35

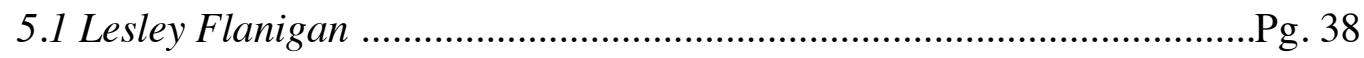

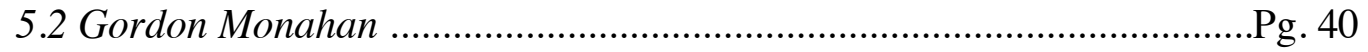

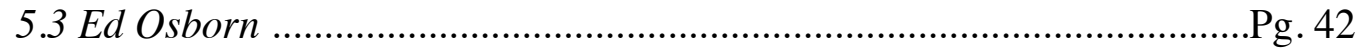

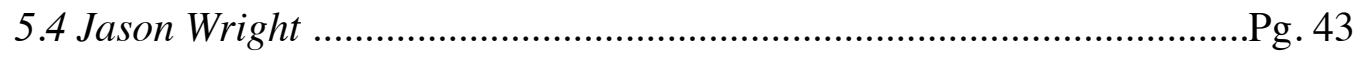


6. Visual Pg. 48

6.1 The Loudspeaker as Visual Artifact Pg. 50

6.2 Sound Art Pg. 52

6.3 Tristan Perich Pg. 52

6.4 Sean Kerr Pg. 54

6.5 Jason Wright Pg. 56

7. Large-Scale Works Pg. 58

7.1 Technological Fetishism Pg. 58

7.2 Sound Art Pg. 63

7.3 John Wynne Pg. 64

7.4 Benoit Maubrey Pg. 67

8. Conclusion Pg. 72

9. Bibliography Pg. 74

9.1 Works Cited Pg. 79

9.2 Figures Pg. 80

\section{Section B: Portfolio}

1. Overview of Practical Work .$P g .83$

1.1 Overview of Sound Material Pg. 83

2. DVD Contents Pg. 85

2.1 Works Pg. 85

2.2 Supplementary Material Pg. 85

2.3 Electronic Copy of Exegesis Pg. 86

3. Table of Figures Pg. 87

4. Live Performance Pg. 88 


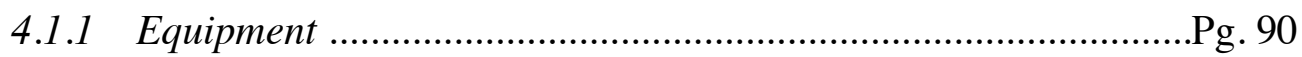

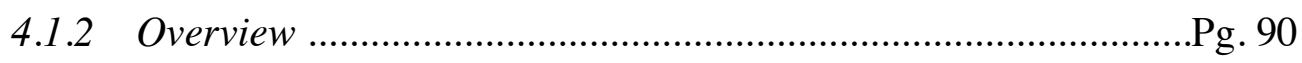

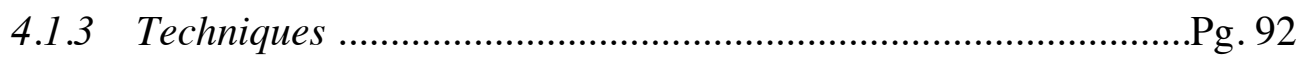

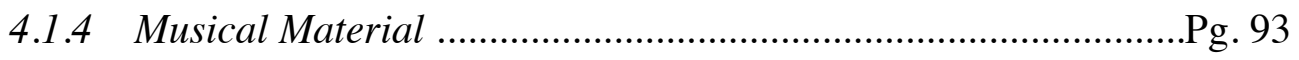

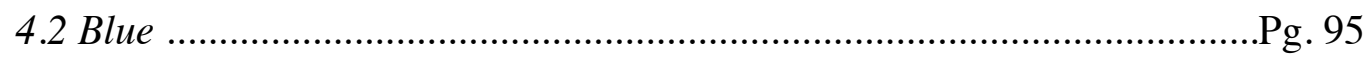

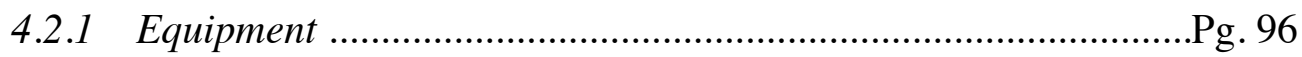

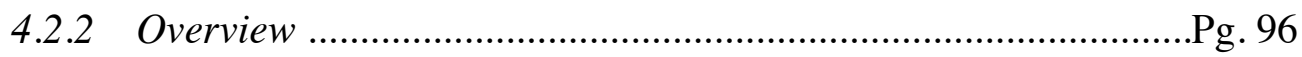

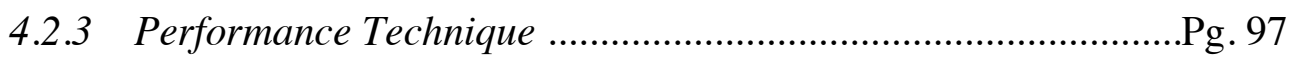

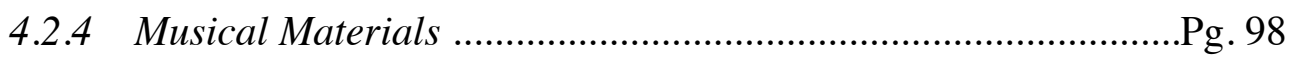

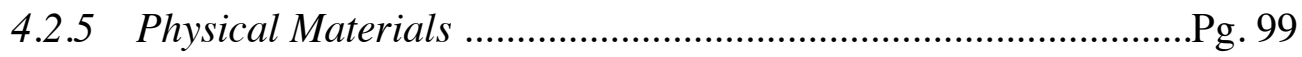

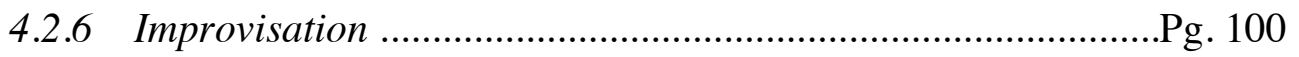

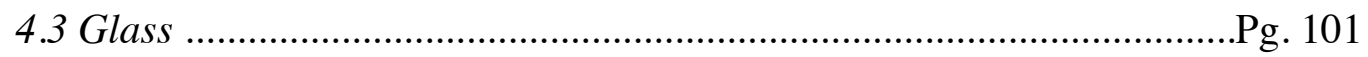

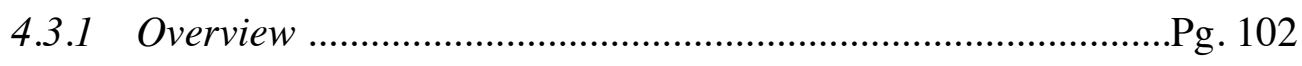

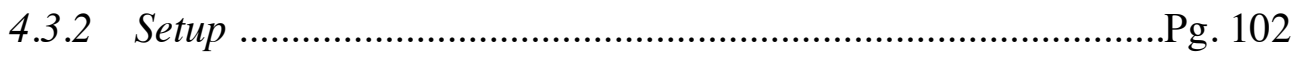

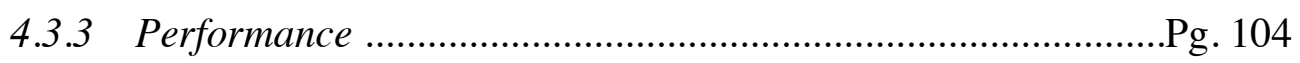

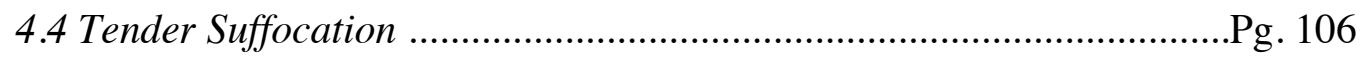

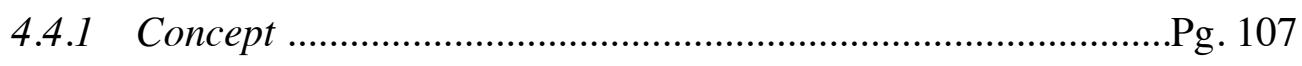

4.4.2 Performance ......................................................................... 107

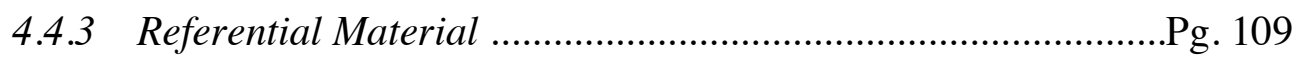

5. Sound Installations .................................................................................Pg. 111

5.1 Deaf of the Author ........................................................................... 112

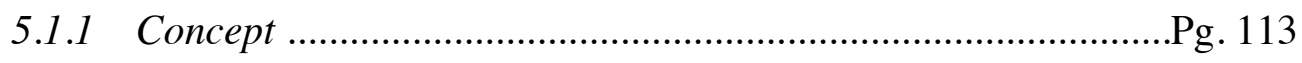

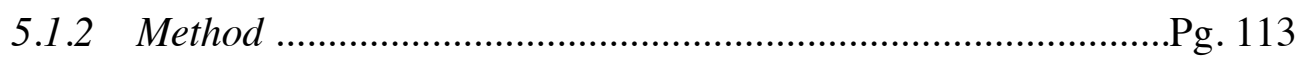

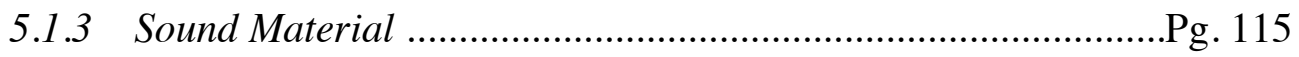

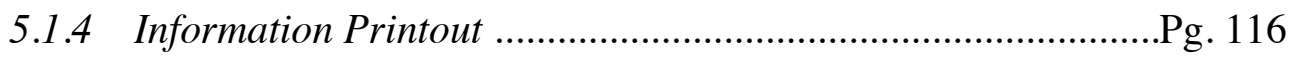




\section{Table of Figures}

Figure 1. Nik Nowak's Soundtank

Pg. 23

Figure 2. Jamaican Dancehall Sound-System

Pg. 30

Figure 3. Lesley Flanigan's Live Performance .Pg. 38

Figure 4. Gordon Monahan's Speaker Swinging (1982) Pg. 40

Figure 5. Ed Osborn's Flying Machines (2001) Pg. 42

Figure 6. Blue (2012) Pg. 44

Figure 7. Dick Raaymakers' Intona (1992) Pg. 47

Figure 8. Transmission Audio's 'In-wall design. Can you see the speakers...?’Pg.49

Figure 9. Sean Kerr's Stacker (2001) Pg. 54

Figure 10. Jukebox Pg. 56

Figure 11. Jean Michel Jarres' Aero Dream One Pg. 60

Figure 12. The Grateful Dead's 'Wall of Sound' Pg. 62

Figure 13. John Wynne's Installation for 300 speakers .Pg. 66

Figure 14. Benoit Maubrey's Audio Igloo Pg. 68

Figure 15. Benoit Maubrey's Temple Pg. 70 


\section{Section A: Exegesis}

\section{Introduction}

An artifact can play more roles in human life than functional ones (Verbeek, 30).

My aim in this exegesis is not to work toward any technological or theoretical developments concerning the loudspeaker. I will seek to explain it holistically as an object, not only as denoted by its primary function, this being sound reproduction, but also concerning its visual and physical aspects, where it moves into the realm of consumer fetishism and becomes an artifact separated from function. I will be exploring the relationship between the loudspeaker and the sound it produces by looking at various contexts where the loudspeaker is appointed differing levels of transparency and thus different perceptual effects. Combining these factors will show how the loudspeaker acts on our perception of sound and the object itself, and how these many facets can be exploited and used in ways that challenge the traditional applications of the loudspeaker.

Drawing on philosophical, phenomenological, technical and theoretical writings, plus an assortment of musical examples, I will provide a framework for understanding the object in terms of these facets. Also addressed will be the way in which both the listener and composer relate to the object and how learned response and expectation play a part in this relationship. I will provide an overview of its use within the sound-based arts, looking at the differing degrees of engagement both the artist and the listener take on and how this is utilised to create the particular work. Through examples drawn from my own practice I will outline potential uses of the object that sit outside established paradigms. 
Another aim is to recognise the loudspeaker as an autonomous object, separate to any sound it may produce. I will be viewing it in light of its intended function, additionally carrying out analytical reductions that allow for concentration on elements of the visual and aesthetics, physicality and commodity. The outcome of this is to showcase the loudspeaker as an instrument in its own right, showing it to have similarities to the traditional definition of instrument in the tactile sense. Furthermore, that because of these elements, it is also instrumental in acting upon the listener perceptually.

Key concepts to be addressed in this exegesis are the transformational qualities of the loudspeaker and the aural and perceptual outcomes of treating the object in a tactile nature. Also under discussion will be the aural and perceptual outcomes of assessing the visual aspect of the loudspeaker both as an autonomous element and together with the use of sound. A foundation for this will be provided in Chapter three, Sound of the Sound where I will outline the modes of listening to be engaged with whilst assessing this work. This will lead to Chapter four, Transformation of the Musical Object, where transformational powers of the loudspeaker will be explored in depth via examples taken from classical and contemporary musical forms. Chapter 5, The Loudspeaker as Instrument, looks at tactile uses of the loudspeaker in the sound-based arts, with examples from myself and other contemporary sound-based artists. Chapter 6, Visual, assesses the loudspeaker as a visual artifact both within mainstream culture and the arts alongside evaluation of contemporary works emphasising the visual dimension of the object. This discussion of the visual is continued in Chapter 7, Large Scale Works, and moves into exploring the elements of fetish and commodity, concluding with examples of sound installation work that exemplify the spectacle through the loudspeaker.

As mentioned above, technical descriptions of the mechanics of the object will be limited. My aim is to move past the loudspeaker's construction, towards considering 
and relating to all its facets within an artistic context. This will be mirrored in my practice-based research where the mechanics of the object are exploited to follow conceptual and aesthetic aims.

\section{Distinctions}

At this point I would like to make a distinction between the object, 'the loudspeaker' and other objects that contain within them 'a loudspeaker'. I would like to make this distinction because the object in question is attributed different sets of affordances and subsequent actions depending on the context of its use. This leads us to differing perceptions of the same object, and in some cases leads us to looking past the object completely, where it may only be part of a greater whole. This distinction is important as it enables greater focus and concentration upon the object 'the loudspeaker', allowing for more specified and detailed observations. Here I will look at a range of uses and contexts in which the loudspeaker appears. Apart from brief references, this exegesis will disregard particular examples where the loudspeaker it is seen to become compliant to a greater object.

This first example in which the loudspeaker is only a part of the object is the telephone. This is a common household item, which contains within it two transducers: a microphone and a loudspeaker cone. In this object, the cone, along with the microphone, is sealed and hidden from view. Because of the physical combination of these two objects within it's casing, we do not see this object as made from individual parts, but as a sum of its pieces. This overall object is the 'telephone'. Through its function it has gained a specific and standardised place in the home and office. The loudspeaker within the telephone is placed within the context of the telephone. 
Subsequently any sounds, such as the voice or various examples of $\mathrm{Muzak}^{1}$, are contained within this telephonic context, becoming perceptually separated from instances when the same sonic object is heard outside of the telephone.

The loudspeaker within a guitar amp is another example of perceptual compliance. The guitar amplifier and loudspeaker amalgam, known collectively as the 'guitar amp', has the singular function of amplifying a guitar signal. This is attributed to many factors including design and the culture surrounding the guitar amp. As a consequence of the branding and imagery linked with the culture, it would be unconventional to use the guitar amp for anything other than its designated purpose. Because of this it is perceived differently from PA loudspeakers at a concert, which are used to amplify the sound emmited from the guitar amp loudspeaker as well as other instruments on stage.

The addition of the smart-phone to the list of standardised social technology has given the loudspeaker a new level of exposure. As a reaction to introversion inducing headphones, new smart-phones come equipped with higher quality loudspeakers, with better bass response and the ability to output sound at higher amplitudes. The use of headphones is discouraged and deemed unnecesary as these smart-phones provide enough amplitude for owners to hear their choice of music above most urban and environmental noises. Although including a high level of multi-functionality, the smart phone is still an object containing a loudspeaker. Likewise, the inclusion of loudspeakers within iPads, iPods, digital cameras and camcorders, alarm clocks, televisions and laptops, fall into the category of containing 'a loudspeaker', thus being perceptually separate to 'the loudspeaker'.

Although the above-mentioned examples in this chapter will not garner any specific focus, the radio will be an exception. The radio is a meta-object consisting of many 
parts. The loudspeaker, as one of these parts, remains physically separated by mechanical and spatial definitions. Because of this I am able to regard the loudspeaker as having its own properties separate to the radio, thus considering it as an object on its own. When generally talked about, radio does not explicitly mention 'the loudspeaker' as what is more important is the content transmitted through the system. As radio is a complex entity, (including but not limited to, the loudspeaker and receiver in the home, radio frequencies, the studio, the DJ and the music itself) I will clarify here what is included when talking about radio and its effect on music. In the context of this paper, radio will include the effects on the music due to the sounding information being transferred via radio frequency, but more specifically the effects the loudspeaker has on this incoming information. I make this reduction here to avoid confusion regarding the many factors involved in radio and to bring focus back to the loudspeaker. Physically, a 'radio' may be an object containing a receiver and loudspeaker in one. I would deem this to be a separate object from a solitary loudspeaker.

I would therefore like to suggest that any physical amalgam containing 'a loudspeaker' is, in fact, different to the isolated object 'the loudspeaker'. The telephone could not operate without the loudspeaker, thus the loudspeaker becomes pliant to the object in direct perception. Even though the reduced physical function of the loudspeaker may be the same, its inclusion within the greater object likens it to prosthesis in submission of that particular object's goal and function. Apart from the radio, these objects and the issues relating to them as far as the loudspeaker is concerned will not be mentioned further.

What I will term the loudspeaker for my purposes here, is: 'An apparatus that converts electrical impulses into sound,2 and which does not rely on physical or perceptual

\footnotetext{
${ }^{2}$ Loudspeaker, Noun. New Oxford American Dictionary 2nd edition (C) 2005 by Oxford University Press, Inc.
} 
inclusion with another object. Although the activation of the loudspeaker requires an amplifier to drive it, and a sound source such as a CD or tape player to conduct the sound information to be produced, these devices remain separate physically and perceptually. Working in tandem with the loudspeaker does not denote that they are included with it.

If an object carries out its function as denoted by a formal description it retains a sense of perceptual continuation, regardless of the size, shape or colour of its physical body. This can be identified as a type/ token distinction. ${ }^{3}$ This also applies to the loudspeaker and can require attention when viewing the vast number of shapes, sizes and materials it can be made in. The differing forms the loudspeaker takes can be seen to deem distinct tasks that are attributed to its particular design. It is true there are many categories of loudspeakers, all designed for a range of functions and locations. This becomes a specific point of interest when an object whose physical function is uniform can be applied in many different ways. Some of these functions include but are not limited to: home stereo, hi-fi, out-door loudspeakers, Public Address systems, studio monitors and car loudspeakers, all of which are made to distinct specifications.

In my own work, two physically differing instances of the loudspeaker are used. Firstly, the object in its traditional state: the 'cone' encased in a wooden or plastic enclosure. Other instantiations of the object consist of varying materials, especially of the frontal cone cover. Many also include a range of number and size of cones. The size of the loudspeaker also differs immensely. The enclosure in this case remains a part of the object, the loudspeaker: whilst functionally acting as a resonator, a stand and protective casing, it also shapes the sound of the object. As the cone mainly remains hidden behind this enclosure, the box is usually the direct visual element we come into contact with.

${ }^{3}$ Stanford Encyclopaedia of Technology: <http://plato.stanford.edu/entries/types-tokens/> (28 May 2012) 
The second type of loudspeaker I will be using, and referring to as simply 'the cone', is the loudspeaker without the enclosure. Through removing the cone from its casing, greater focus on the mechanics of the object is achieved whilst still retaining its function. These two instances of the object will afford different perceptual and aural outcomes depending on whether they are encased or not. An identical sound played through the same cone will be altered significantly due to properties of the physical body of the cone and enclosure independently. In a descriptive sense, these two instances of the loudspeaker both retain their essence and function, thus removing the cone from the enclosure is not necessarily a destructive act, but more a means to conceptual and aesthetic ends.

\section{Sound of the Sound}

Him slide the switch and the bass just swell, him win the session because of that, because of the sound not because of the record (Henriques, 86).

In of some of the works to be described, as well as in my own practice-based explorations of the loudspeaker, sound verges on being reduced to a physical activation mechanism. As a subservient element, the role of sound in some pieces is to draw out the conceptual component of the work, rather than allowing focus on the sound itself as an object and point of interest. In some cases, such as works that engage in tactility of the mechanism and physicality of the loudspeaker, the focal point for the piece is not just the sound itself, but also the tempering of these tangible elements.

Within the work I am discussing, a shift in the mode of listening may be required. As detailed by Michel Chion, the two main modes of listening we engage in on a day-today basis are causal and semantic listening (Chion, 1994). Simply put, we listen to 
determine the source of a sound and seek to extrapolate any meaning or message it may carry. To engage in the 'sound of a sound' one may have to undertake what Pierre Schaeffer termed 'reduced listening', whereby causal and semantic listening are temporarily suspended to determine intrinsic aspects of the sound, such as temporal morphology and frequency components. Examples of this type of listening are found in both sonic and sound art practices.

Schaeffer's experiments in musique concrète saw him using 'everyday' sound as raw musical material. In using everyday sounds, Schaeffer was aware of the overbearing instinct to listen to sounds with a focus on the cause of the sound or the message it may carry. A famous example of this is the steam train used in Etude Aux Chemins De Fer (1948). Instead of listening directly to the source, Schaeffer was more interested in the intrinsic properties of the sound, such as the rhythm of the wheels, frequency content and the morphology and development of sound over a particular sample. This reduced listening required a meditation upon the sound until the source disappeared, giving way completely to the sound of the object. To achieve this, the context of the sound becomes all-important. By framing the sample as a musical object within the context of a piece of music, Schaeffer's aim was to encourage listeners to engage in a reduced, specifically musical, listening. His hope was that they discard or move past the other two forms of listening to hear past sound, towards music.

My own work encourages another form of reduced listening: technological listening ${ }^{5}$, albeit in a different sense originally intended in the term. This term was coined by Denis Smalley, and is described as occurring "when a listener 'perceives' the technology or technique behind the music rather than the music itself" (Smalley, 2001). In the context of his paper on spectromorphology, Smalley believes 'the technology should be

\footnotetext{
${ }^{4}$ See Chion, 1994

${ }^{5}$ Smalley, 2001
} 
transparent'. He immediately stresses that the schism between technology and music can open up due to this type of listening. Consequently a listener may arrive at a point where 'musical meaning is blocked' (Smalley, 2001). Marc Perlman takes this sense of meaning a step further and transitions into more personal and existential territory, where he relates the experience of an audiophile dealing with this technological split:

The temptation to analyze can distract the audiophile from proper emotional selfabandonment, as is clear from this confession in a letter to the audiophile press: 'As hard as I try to ignore the sonic quality when listening to an audiophile record, I can't help but sometimes lose the music (you call it the "emotional experience") because I'm focused on the sonics (Cohen, 1993) (Cited in Perlman, 2004).

The way I am framing technological listening here does not impair the listener's engagement with the sound as Smalley would argue, but it is used to heighten a sense of causality sourced in the technology itself. The sound of the technology and the sound of the sound created by the technology are both equally important. Although these aspects may be hard to distinguish within one example, they can be compared and analysed over many points of reference and many instances of a particular technology, in this case the loudspeaker.

In certain facets of popular media, technological listening becomes a condition of engagement. With the revitalisation of the vinyl market, and to some extent cassettes, music listeners and vinyl aficionados engage in the sound of the medium, viewing it as paramount to their experience of the music. The next chapter will explore these two objects: the physical medium and the music. Though fused physically, these objects can be separated in the consciousness of the listener to reveal the sound of the music, the sound of the medium and the effect of the medium upon the music.

Historically, this sense of sonic disconnection was brought about as playback technology propagated in homes. As the demand for such technology increased, not 
only did this lead to consumerist fetishism (which will be explored in Chapter 7), but to a place where the quality and range of the technology differed drastically. As a consequence of this, a disconnection was established between the sound originally recorded, and the sound of the music played through this technology. The musical object in this instance lost its sonic consistency and was left open to transformation and interpretation via domestic technology.

\section{Transformation of the Musical Object}

Sonic perception is variable; we fill in and compensate, we adjust (Whitelaw, 2).

The hi-fi re-speaks the recorded artifact, filtered through its electro-acoustic peculiarities (Whitelaw, 3).

My concern in this chapter is to address the concept of the musical object and explore how the loudspeaker has the ability to transform this object.

Through the loudspeaker we may receive drastically differing aural information about a musical object from various instances of the technology. Under closer inspection we may recognise the changes to the musical object as a consequence of mediation, but more generally we make a move to reconcile these changes towards hearing the intended sound.

For my purposes here, I will outline a recording as a musical object, and I will define the musical object as so: An idea/ information floating in the ether, un-materialised until it is pushed through the loudspeaker. Whether in an analog or digital medium, it exists much like the score, an idea and an ideal, until it is realised. 
When one mentions a recording we can deduce data gathered, existing in some form, with the potential to be realised in some aural degree, necessitating the use of a loudspeaker. This object, separate from the 'piece of music' it may represent, is an object of fixed media that only exists in one preferred state. This state, being one that either the composer, performer, audio engineer or a combination of these decide on. In short, any instance of sound that is able to undergo exact repetition ad nauseum.

Patricia Carpenter says of the musical object: 'A piece of music is not only an object grasped, a perceptual form; it is an object heard, grasped in a specific way' (Carpenter, 69). Determined by factors such as time, place and mood, our interpretation of the same musical object may vary, allowing us different portrayals each time we hear it. As important as these factors is the technology used for us to hear the said music, as it significantly informs our 'grasp'. Don Ihde makes an important point about technological bias, stating 'each different technological system transforms the quality of sound and music heard in a different way. No system attains perfect transparency and each produces a different selectivity of sound qualities. There is no neutrality to recording technology' (259). Following Ihde, my argument here is that auditioning the same piece of music over various technologies results in differing musical objects. As Arved Ashby asserts, 'technologies of depiction have brought about a fragmentation rather than a consolidation of the musical self' (196).

To fully appreciate this we have to attend to the role of music technology in opposition to the idea of a piece of music as an immutable fact. This does not mean technological changes are detrimental, but that they are a conditional factor of the use of technology. The transformation that I am suggesting takes place is essentially spectral due to the physical factors of the loudspeaker, the body and the cone. Not only does the loudspeaker transform the musical object, so too do we listeners in the nature of our 
interpretations. As Merleau Ponty puts it 'Judgement is often introduced as what sensation lacks to make perception possible' (Ponty, 37). Perceptual and physical changes, attributed to both the technology and physical condition, lead us to either hear the sound of the loudspeaker or make cognitive amends to the musical object to make it whole. Ihde provides an example of this phenomenon when addressing his own hearing loss:

For example, if I listened to music, if I didn't know the piece, I could not be aware of what I was not hearing (for example, the higher frequencies), but, if I listened to a piece that was familiar, I could quite distinctly become aware that I was missing what I remembered hearing in my listening past! (247).

From here one can deduce that the perception of sound gained by the listener may at times end up being a judgment based on audible input, rather than direct sensory decoding.

\subsection{Embodiment of the Object}

The fact that the musical object changes through technology may be a simple observation, one that may seem obvious when attention is brought to it. But to set up this school of thought in opposition to the traditional view of the embodied and subservient loudspeaker, sets up many affordances in terms of performance and installation. When taking into account this idea of transformation it may help the listener to render information about the works that would not normally be seen or heard. The relationship of embodiment undertaken with the loudspeaker in an everyday context sets up regulations restraining perceptual engagement as Philip Brey details:

In embodiment relations, the embodied technology does not, or hardly, become itself an object of perception. Rather, it 'withdraws' and serves as a (partially) transparent means through which one perceives one's environment, thus engendering a partial symbiosis of oneself and it. (Brey, 3) 
The cognitive acquisition of transparency is indeed a learned skill, one not only cultivated when music is technologically mediated, but certainly when auditioning acoustic instruments as well. Retaining consciousness of the visual and mechanical aspects of the technology may require as much practice. Audiophile culture provides an example where transparency of technology is substituted for complete incorporation into the listening event. In this instance the visual presence of the technological object is accentuated for reasons seemingly unrelated to sonic function, that is, reasons of status, consumerism and fetishism. Elements of fidelity and personal aesthetic are accentuated but the affordances of the technology remain stark and rigid.

In some cases the loudspeaker does become an object of direct perception where there may be no chance of withdrawal, as it becomes symbiotic with an external object. The affordances here extend beyond the capabilities of the stereo system as the possibility of spatial and spectral change are incorporated into the system. 


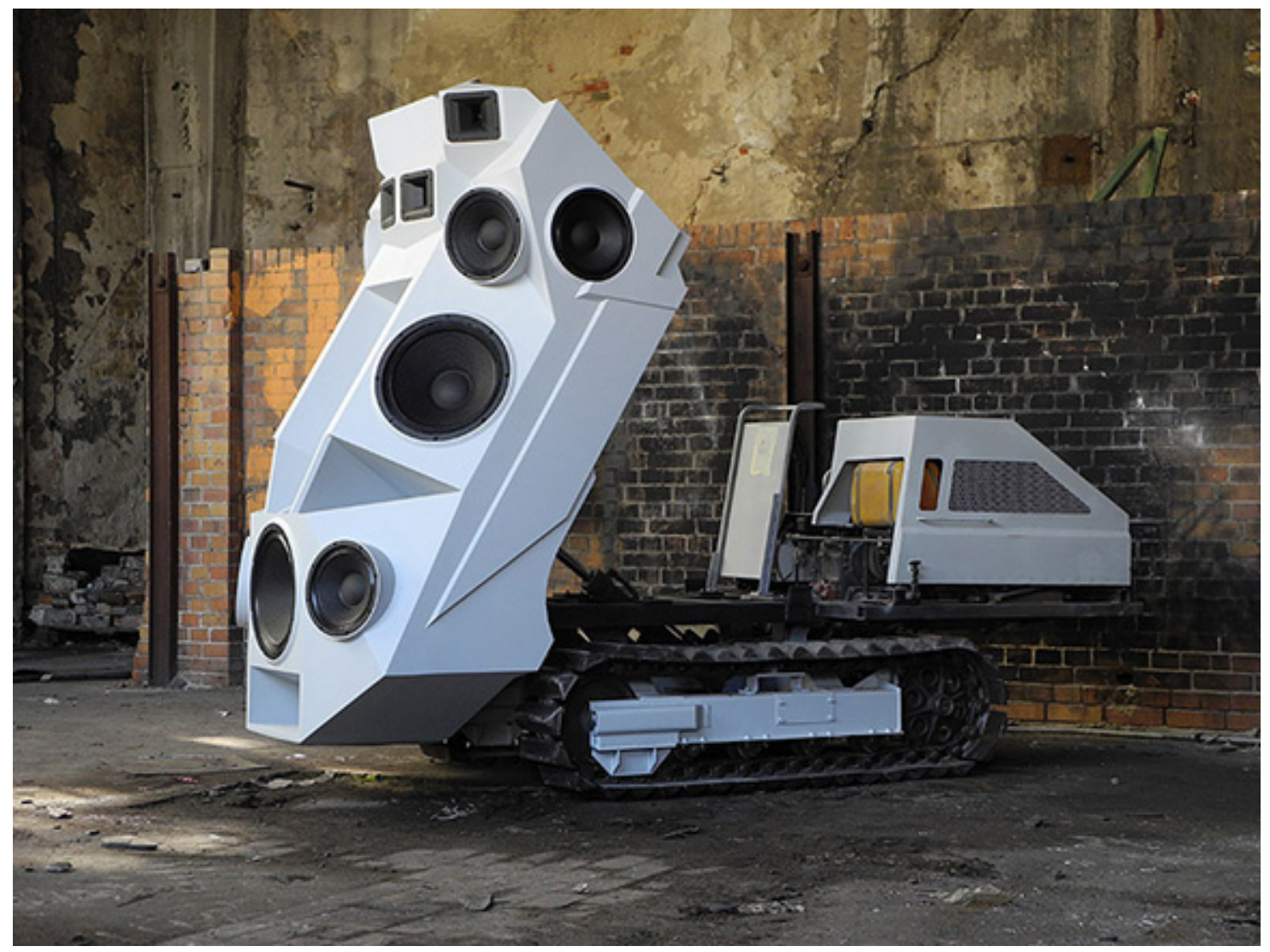

Figure 1. Nik Nowak's Soundtank

Nik Nowak's Soundtank is a custom-built sound system vehicle; it boasts eleven speakers and '4,000 Watts of pure Hi-Fi assault' (Nowak, 2011). Complete with microphone, mixer and samplers, this machine is an example of extravagant design relying on the spectacle it creates to draw an audience's attention. In this particular case an interesting dialogue is setup between the Soundtank and the musical object where the musical object is changed due to the extreme nature of the machine. The music and the machine have to fight for the listener's attention due to amplitudinal and visual excess. Sensory focus becomes overwhelmed and it is unspecified as to where the audience should focus. The musical object is changed spatially due to the convex setup of the loudspeaker over the surface of the tank. External movement of the Soundtank also causes spectral and spatial change, due to filtering, as it moves in relation to the audience. In this example, the musical object is changed not only sonically, but also perceptually. 


\subsection{Jukebox (2011)}

My sound installation, Jukebox, addresses the perceptual change of music due to the technological object. Jukebox is a loudspeaker sculpture, consisting of forty-six loudspeakers stacked to create a wall. These loudspeakers are setup in series, connected to a single stereo amplifier, so they can all receive the same audio information simultaneously. The speakers used are of varying shape, size, material, quality, colour and age. My aim in this installation was to bring attention to the object and the effect it has on intrinsic qualities of music auditioned over it. Allowing the listener to audition music (which was chosen by the audience and brought in on the opening night event), over the various loudspeakers at the same time encouraged this, as the presence of the object itself demands to be addressed and comprehended alongside the music. The visual and audible information combine to inform a particular perception of the music played. As with Nowak's work, the sonics of the musical object are changed, but more important may be the way the listener is encouraged to engage with the musical object. By placing these loudspeakers next to each other, the work gives the audience the opportunity to audition and compare each of the loudspeaker's particular characteristics, both visual and aural, and create their own conclusion as to whether the sound changes or not.

Also addressing similar conceptual concerns is my installation Deaf of the Author (2011). A full explanation of this work can be found in Section B, Chapter 5.1 of this exegesis.

\subsection{Theodore Adorno}

Theodore Adorno offers further enlightenment concerning the transformation of the musical object in his essay The Radio Symphony (1941). Using the example of Beethoven's Fifth symphony, he explains the perceptual change a symphonic work goes 
through when transferred via radio to the listener. Adorno considers the intrinsic aspects of a symphony such as timbral, spatial and dynamic changes. He proposes that these aspects are crucial to a works realisation and when alteration of these factors transpires, especially as drastically as may occur over radio, this means that the work ceases to be a symphony and becomes a 'mere chamber work' (Adorno, 257). For Adorno, engagement with technological listening dilutes musical presence and intention. The problem in this case is not achieving technological transparency, but the concern to avoid the dilution of music through mediated listening.

Evan Eisenberg addresses the matter of balance, concerning spectrum and amplitude of absolute music, when he examines recording in the studio. Contrasting a recording with a performance, Eisenberg sets up the possibility of surveying these two sides of the work individually:

Such matters as recording balance may seem trivial from the point of view of art. What difference can they make to the whopping power that marks a great performance, that only the artist can provide?... Matters of balance were important to Prokofiev and Stokowski, and both knew at least half the action was in the control booth. Balance affects the listener's perspective, determining how and where the music shall draw him in. On some interpretations of music, the very ethics and metaphysics of a piece may depend on its balances (Eisenberg, 100).

The repercussions of the changes afforded by the medium in this case are severe. The danger in this case is not aimed toward learned listeners who have heard a work in its unblemished state, but rather to those who are in a position in which this radio listening provides telling information and allows a solidification of the musical object in ones mind. 'The idea of a piece of music carries the requirement of some kind of wholeness' (Carpenter, 58), and if this wholeness is disturbed and one is provided with an incomplete picture it may be more difficult to reconcile the real object with the object thus provided. Comparisons of the work told by learned listeners and by amateur ones 
may provide varying results, not just in the language or articulations, but in a wider sense: emotionally as well as intellectually. As Adorno relates, 'What is heard is not Beethoven's Fifth but merely musical information from and about Beethoven's Fifth' (Adorno, 262). Radio and the surrounding technology fail to inscribe the many intricacies of a work. This leads to an impaired judgment of the said music and is taken lightly in many cases as described above. However, Adorno relates with solemnity the dire outcome of the symphonic work attributed to technological mediation.

When comparing the technologically mediated rendition of a work to Adorno's 'perfect' rendition, many intrinsic sonic dimensions are affected. Fidelity in particular is called into question as recording technology; microphones, mixing equipment and loudspeakers have an impact upon the spectrum and quality of the work. Amplitude, due to a listeners' active control over the volume impacts the overall dynamics of the work. The issue of spatiality and the encompassing effect of the concert hall, including the room acoustic, may not be captured, impeding awareness of the space of the hall and size of the orchestra. Whilst this last example may not hinder musical understanding, it may lead to the negative transformation of extrinsic factors such as temporal and physical envelopment, which for Adorno, are as important in the understanding of the work as the intrinsic factors.

\subsection{Leopold Stokowski}

Taking a stance close to Adorno but with a very different outcome, conductor Leopold Stokowski addresses the musical work and its potential to affect the listener. This is done concerning the intention of the composer and Stokowski's own interpretation of the work. As with Adorno the repercussions of technological inaccuracies are severe, with the very substance and 'soul' of the work being subject to alterations. These consequential outcomes rest on many factors, some more closely related to the 
performance than others. Stokowski realises the work does not end when the physical performance does:

Stokowski's abiding belief...was that the total intellectual-emotional-spiritual impact of music depends not only on the composition, performers, and conductor, and on acoustic properties of the performance and the audition environment, but also on the technologies with which the music is performed, recorded, transmitted, and reproduced (McGinn, 53).

Where Adorno and Stokowski diverge on this matter is with Stokowski's ability and desire to infer further authorship upon the work independent to the technology. As a conductor 'Stokowski was renowned or...notorious for his interpolations, changed dynamics, re-orchestrations, and even changed notes, always with a view to increasing the effect of the music on the audience' (McGinn, 44). For Stokowski, the musical object is not as immutable as Adorno would have it be. Ultimately attempting to accentuate the effect of the music upon the audience, Stokowski sees technology as part of the musical-interpretative process. Rather than provide an unblemished rendition of the original work, Stokowski uses technology to create a personalised mark upon it.

\section{5 'Beats'}

In a more modern example of authorship, listeners can use technology to fabricate their own version of music. While the choices available are limited and the level of control is not to the extent Stokowski may have had, surveying the habits of listeners and understanding what the technology means in this context is important. The headphones, beats by $d r . d r e^{6}$, created by Hip-Hop producer Dr. Dre are marketed with this statement:

People aren't hearing all the music. Artists and producers work hard in the studio perfecting their sound. But people can't really hear it with normal headphones. Most headphones can't handle the bass, the detail, the dynamics...With Beats, people are going to hear what the artists hear, and listen to the music the way they should: the way I do (Dre, 2011).

\footnotetext{
${ }^{6}<$ http://www.beatsbydre.com/Default.aspx $>$ (28 May 2012)
} 
Modern reproduction technology becomes about instilling a personal character onto the music one listens to. Mark Richardson depicts this in a comment about beats headphones: ““The way we hear music.” It's personal, right?' (Richardson, 2012). Richardson continues:

Beats by Dr. Dre are popular because they don't reproduce music as much as they transform it. They are the right headphones for the current era, because their design "customizes" the sound for the listener who wants bass. Music is never finished; we can chop and screw, add bass... And people will buy headphones that finish the music in the way they like (Richardson).

This characterises the mindset of modern audio technology consumers and producers, where the mix of the original work is left behind in lieu of something more personalised. With beats, Dr. Dre allows for the listener to hear 'his' productions reproduced accurately. Consequentially, any other music reproduced by the headphones will also undergo transformation. Essentially, a response to treble orientated technology, beats and other similar technology (customised subwoofers in modern cars are a prominent example), seek to redress spectral imbalance by filling in the low end and engaging with the listener in a more haptic sense, so that one can 'feel the rumble in (your) cheekbones' (Richardson, 2012). Showing similar concerns to those expressed by Stokowski, beats place importance upon the experience of the music, not necessarily the need to retain intrinsic elements of any particular work as defined by the composer.

\subsection{Bell Telephone Laboratories}

The issue of technological transparency is evident in Stokowski's collaboration with Bell Telephone Laboratories between 1930 and 1940. This collaboration sought to address qualms concerning technology similar to Adorno's own, through experiments to increase the fidelity of both recording and reproduction technology. This represents an 
attempt to retain the essence of the work while also increasing the effect of the music upon the listener. During this collaboration, Stokowski said this:

There is a very mysterious thing about music. It is psychic suggestion. That is, to my mind, the most important part of the suggestive power which can carry us into the most remote spheres and realms of feeling and thought, and things that are higher than feeling-that is the important thing about music. And in order to be able to do that we must have this greater range which we have had demonstrated here tonight, greater range of frequency, of volume, and elimination of foreign noises, needle scratch, static and all those noises that we hear in radio (Bell Laboratories Record 10 (January 1932): vii-viii) (McGinn, 52-3).

The outcome of this collaboration saw Stokowski 'unleashing his electrical thunderbolts' (McGinn, 59) in a demonstration of the finale of Wagner's Die Gotterdammerung. McGinn quotes Stokowski as saying that 'Wagner himself might have imagined such splendor and power of tone, yet was never able to realize it with the means at his command' (McGinn, 59). This suggests that technology has the ability to transcend the imagination of the composer and the abilities of performer. Stokowski takes the work and enhances and intensifies it to such a degree that to realise the work again without these technological revisions would be as much of a failure as to audition it over broken loudspeakers. Whether this collaboration was successful or 'merely another attempt to gratify [Stokowski's] insatiable ego' (McGinn, 47) is irrelevant, as what is shown is the possibility of altering the musical object ad infinitum with the aid of the right technology.

Auditioning Beethoven's Fifth over a range of technology, from lo-fidelity to hi-fidelity audio equipment, allows us the possibility of access to completely differing renderings and thus interpretations of this particular symphonic work. In addition this allows the application of this idea of technological transformation to all mediated sound, where assessing the purpose of the sound is as important as how the sound is reproduced. What can also be understood through this, is that there exists a correct medium for any musical work, at least when remaining true to the intentions of the creator of the music. 
For Adorno, the symphonic work must reside in the concert hall, in the same way that at the same time Jamaican dancehall music requires the specific use of a Jamaican dancehall sound-system. In taking a medium-specific approach to correctly replicating a piece, music requires both a certain physical placement and access to any idiosyncratic technology, otherwise the music becomes transformed and ceases to be what it was once denoted as.

\subsection{The Dancehall Sound-System}

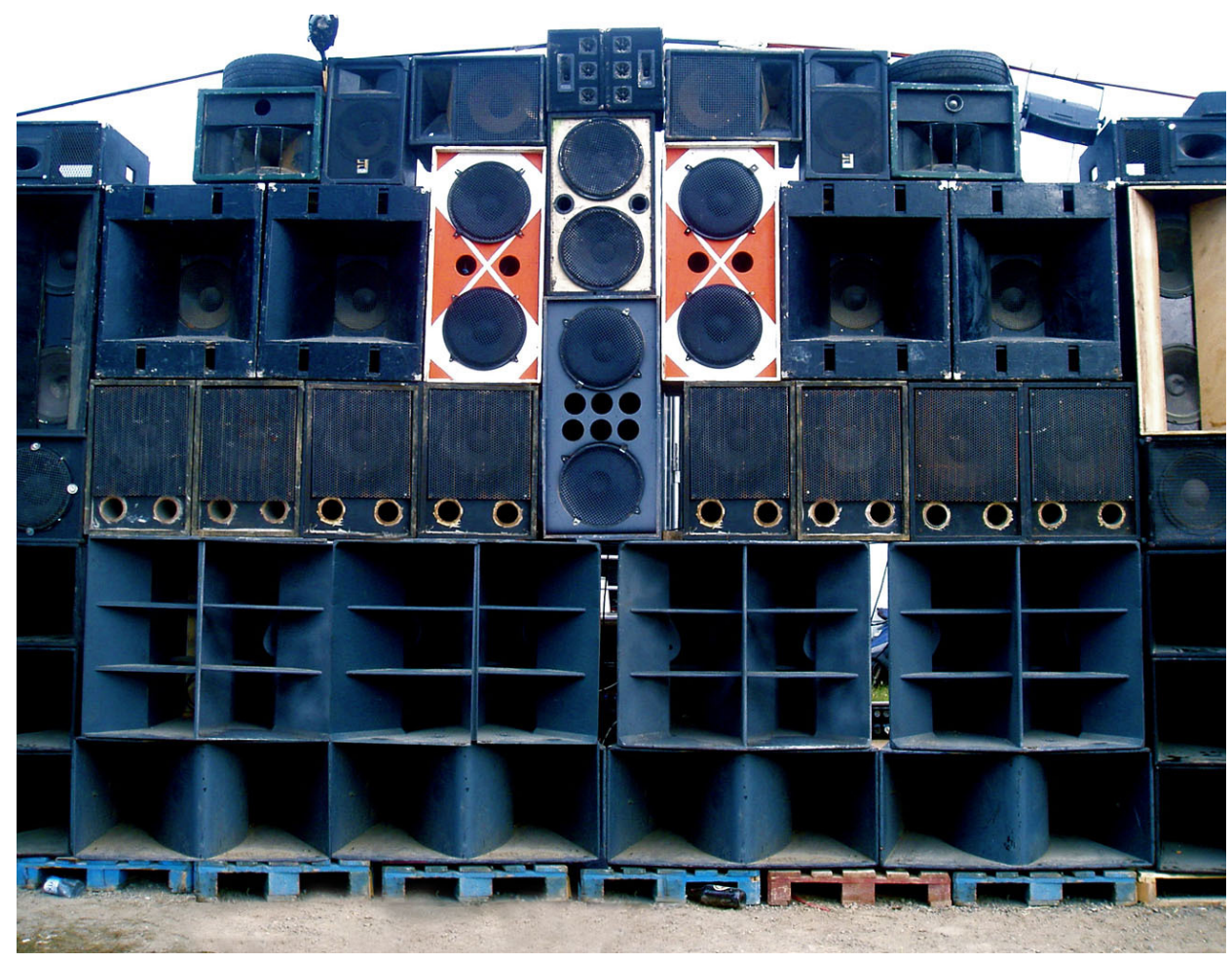

\section{Figure 2. Jamaican Dancehall Sound-System}

An example where the role of loudspeakers is made explicit and is indeed recognised as a transformational tool and instrument can be found in Jamaica in the form of the Dancehall Sound-system. Where many applications for the loudspeaker seek to disregard its effect on the musical object for the want of transparency, it is precisely this transformational effect that is to be exploited within this context, as detailed by Julian Henriques: 
A sound system is a phonographic instrument...It generates sounds by the reproduction, or re-presentation, or re-playing of already-recorded music, as with a hi-fi system...This is indeed most important: each sound system gives its particular mix to the already-mixed record. Unlike a P.A. (public address) system or stage show set of equipment, the absence of a "live" performer concentrates attention on the qualities of sound itself, as distinct from those of the original musical performance, or the production or post-production of the recording (Henriques, 48).

Here the proprietors of the style set up the particular distinctions between the components of the system straight away: the technology, the music, the resulting musical object and the sound of the music are all recognisably distinct. The recognition of the sound system as instrument leads to an open discussion on how it can be used and what can be achieved through it. No longer a means of mere reproduction, the sound system exists as an integrated object that creates music. This straightforward approach to recognition of these different parts of the chain is unique. The users of this technology take the transformational power of the system as an accepted outcome. This is essentially an example of remix culture where the remixing is done instantaneously through the technology in front of the audience. In this case, fidelity is not as important as experience. The purposes of the original works are subverted and new purpose is brought out through the technology.

\subsection{Musical Purpose}

When loudspeakers are setup insufficiently within a shop or venue, the ability to replicate the musical information of the original work is reduced. This creates a sonic link to the 'Treble Culture' of the 1970s, which has 'since returned with the technical limitations of mobile device earphones for delivering lower frequencies effectively' (Henriques, 54). Hearing the same work again on an adequate system gives the piece a whole new meaning. It accentuates the purpose of the track. A dance music track without the bass frequencies replicated sufficiently, does not become a dance music 
track and may not invoke dancing, until it is realised to its full potential. Henriques details with regard to Jamaican dancehall systems:

What pulls the crowd towards the sonic event of the session is their anticipation of its visceral sensory pleasure - the most powerful music machines in the world vibrating every organ of the body. The crowd experiences such cellular intensities as the sheer immersive weight, liminal force and substantive presence of the sounding - impossible to escape or deny. The audible becomes haptic and the intangible tangible. This fills brim-full the "bowl" of the dance-floor between the stacks of speakers that would normally be considered only as an empty "space" (Henrique, 53).

It is increasingly apparent that technology can be inimical to the purpose of a form of music. This however has not created a move towards realising and fulfilling these purposes. Rather, technology advocating accessibility has been pushed to the forefront of consumerism, favouring availability over fidelity. As an example, if one were to audition noise artist Merzbow over laptop loudspeakers, only one particular angle of his work may be heard, leaving other facets unheard and unexplored. Loudspeakers of insufficient size and limited frequency response may cause dilapidation of amplitudinal envelopment, which can be crucial to experiencing noise music. Providing a transcendent moment for the listener due to complete physical envelopment, as is the case with Jamaican dancehall music, can be paramount for a holistic experience of the music:

Masami Akita (a.k.a. Merzbow)...believes that all music is a sadomasochistic act...in his concerts he uses noise levels of over $110 \mathrm{~dB}$, well past the pain threshold of the listeners. So he makes the sadomasochistic relationship between audience and performer quite obvious, as it becomes physical. Bass sounds really "touch" the listener (Friedl, 30).

This said, unconventional listening habits might provide a totally different experience of the music, one which may not be gained from auditioning the music in the preferred environment. As detailed by Verbeek: 
Technological artifacts indeed close off some possibilities by the way they mediate experience, but they also open up new ones. Technology mediates our behavior and our perception, and thereby actively shapes subjectivity and objectivity: the ways in which we are present in our world and the world is present to us (Verbeek, 203).

My music of choice when I read on my laptop is frequently Merzbow's album Merzbuddha (2005). When played at low amplitude, only the higher frequencies in the spectrum are audible creating a type of fluctuating static. This ends up being very relaxing and pleasant, affecting my ears far differently than if I were to play the same piece on larger loudspeakers. One may argue, following Adorno, that what I am hearing is not Merzbow, but merely musical information from and about Merzbow. My choice of auditioning this music on a laptop causes a disjuncture of both aural outcome and purpose of the music, submitting the musical object to my will as listener. Eisenberg expands on the idea of musical purpose, detailing the negation of intention due to music's disposal by the phonograph, and other portable technologies; 'Anyone who owned a phonograph could use music for whatever he chose, in cheerful contempt of the composer's intentions, cultural conventions, and the sacred Geist or spirit of the music' (Eisenberg, 39).

\subsection{Visual analogy}

One example of an artist attempting to retain and reclaim ownership over specific properties of the artwork, which is to be mediated by technology, can be seen in the DVD release of David Lynch's movie Eraserhead (1977). At the insertion of the DVD into the player, a message appears before the menu screen detailing the use of the brightness function on the particular TV used:

Typically, television sets are not set up properly when you take them out of the box. They are setup too bright by the factory in order to compensate from the bright light in store show-rooms. This will not give you a faithful reproduction of films and television programs that you watch on it. The calibration procedure here will set up your TV for Eraserhead and any other programs as well (Lynch, 2002). 
The next menu-screen details a procedure whereby the brightness is turned right down, then brought up slowly to reveal a hidden image. When the image becomes visible, this is the correct level of brightness and will provide you with the 'faithful reproduction'.

The move to towards a more 'correct' rendition of the movie may be lost if the TV screen is set too bright. The intrinsic property of brightness in relation to the screen and the room is integral to the experience of this work and is not a matter of chance but a distinctly composed element. Viewing the movie with the picture either under or overexposed diminishes the original detail that the artist would seek to preserve. Through this message, Lynch implies that the essence of the work may suffer due to incorrect use of the technology. Apart from preserving the work, this message serves to engage the audience member more thoroughly into the film, bringing into consciousness the particular element of brightness and how this has an effect on the work.

In this chapter, the loudspeaker was shown to infer authorship upon the musical object. The next chapter will address the possibility of generating new and original musical objects as the loudspeaker is placed in performative contexts and subdued to tactile modifications by a performer. 


\section{The Loudspeaker as Instrument}

The "player" of the microphones is more of a musician, in the sense of a performer, than a lab technician or a surgeon (Raaymakers, 2008).

Throughout the evolution of the loudspeaker, the fact has remained constant that touch is taboo. A faulty loudspeaker is discarded, unable to fulfil its function. The cone, paper or plastic, is fragile, and tampering with it is among the fastest ways to impair the object. Rips, tears and holes in the cone spoil the potential for perfect reproduction. Even more fragile than the needle and the vinyl record, the hands on approach developed for the former is not suited towards the dignified box. This notion of restricting touch characterises the attitude toward the loudspeaker in nearly all facets of its use; it is only recently within sound based arts that the tactility of the loudspeaker is beginning to be explored. This change in attitude has already been made evident in many aspects of musical technology, where malleability and impermanence of the physical mechanism are exploited for creative ends. ${ }^{7}$ But the loudspeaker has been one aspect of the electro-acoustic chain ${ }^{8}$ that has, until now, remained fairly untouched.

The visual component of performance provides considerable information for the listener and is intrinsically linked to an understanding of the mechanics of the pieces I will discuss. This visual element is only addressed briefly in this chapter, as it will be discussed in depth in Chapter Six, Visual.

To set the scene in terms of creating a transition from a technologically nonparticipative standpoint to an instrumental one, I will outline Dick Raaymakers ${ }^{, 9}$ music-

\footnotetext{
${ }^{7}$ See for example Caleb Kelly's book Cracked Media (2009)

8 This chain can be typified as the signal path a sound takes from the source, passing through transducers and any combination of audio technology, to the ear.

${ }^{9}$ Alternative spelling: Raaijmakers
} 
theatre piece, Intona: dodici manieri di far tacere un microfono (twelve ways to make a microphone shut up) (1992). Attention in this piece is directed at the microphone. What is shown in this example is the possibility for technology to transition from being compliant to an instrumental element within the composition. In this example the importance is placed on the way the technology is framed. The apparatus, in this case the microphone, is transformed into an instrument: "the "microphonist" [becomes] an "instrumentalist", (Raaymakers, 2008). Raaymakers integrates the object into the compositional stream of the work. By boiling, dismantling, sawing, crushing and dissolving the microphones, whilst recording their sonic output, he creates a work in which the audible component cannot be realised without the physical alterations forced upon the objects. This example is also important due to the fact that it alleviates the user's concern to keep the object intact. Where traditionally the microphone and loudspeaker are discarded when broken, recent artistic practices show that this malfunction may be accentuated to create music, as the Raaymakers example demonstrates.

The potential for the loudspeaker to be developed as an instrument in its own right, playing an active part in the morphology of the sound fed through it, has begun to be explored. The idea of using the loudspeaker for generation, not just reproduction of sound is put forth by David Tudor:

After all what is a loudspeaker? At present it's a reproducing instrument, but my feeling all along has been that you should regard it as a generating instrument... Why shouldn't there be a thousand or more ways of building loudspeakers?... Suppose you build one which only responds to the frequencies between 100 and 200 ?... If you put sine waves through it, then you get quite a different sound emerging... The loudspeaker is transforming what goes into it, instead of reproducing it (Tudor, 1972, 26) (cited in Emmerson, 2000, 161).

What ensues is not unlike the transformations undergone with the emergence of the record-player-as-instrument at the origination of Hip-Hop culture. The DJ using the 
record player and surrounding technology (mixer, amplifier, loudspeakers, audio effects) to manipulate the intrinsic parameters of the music gives the possibility of creating a unique musical object. Manipulating the parameters of EQ, pitch and speed, creating flux in the gestural and temporal outcomes of the piece is directly similar to the way changes are undergone the musical object through the Jamaican Dancehall system mentioned above. In my own experiments and the experiments of other artists, which are referred to in this chapter, utilisation of the loudspeaker affords many similar manipulations and transformations of the musical object. Of note at this point are the important differences between the loudspeaker and the record player and their respective places in the electro-acoustic chain. This being the fact that the vinyl itself, as the medium and source of musical information, lies at the beginning stages of the chain, whereas the loudspeaker lies at the end of the chain before the sound reaches the ear. It is at this point where electrical energy is converted into acoustic energy that becomes audible. Applying the sense of touch to the loudspeaker here affords other types of manipulation and means that changes made to the output of the loudspeaker are directly physical rather than the result of internal electrical or digital modifications. While further manipulations to the audio may occur earlier in the electro-acoustic signal chain, my focus in this chapter is the tactile nature in which the loudspeaker can be approached to transform sound, either audibly or perceptually.

Here, five different artists and performance methods are explored with each piece demonstrating how tactile tempering of the object extends its use, whilst adding perceptual and aural layers to the work not otherwise gained through conventional usage. Exploring and utilising the full capabilities of the loudspeaker expand the function of the object in a way that brings it closer to Tudor's idea of a generating instrument. 
Five types of tactile modifications are detailed below, both in performative and sound installation contexts: firstly, touch with the performers hand; secondly, composed spatial movement attributed to performer movement and linking apparatus; thirdly, controlled spatial movement within the constraints of a sculpture; fourthly, placement of objects upon the loudspeaker with examples of the peripheral objects both remaining static and being used more actively; lastly, two examples are listed where the audible output of the system is secondary to the physical task set for the loudspeaker by the composer. One of these examples shows how the movement of the loudspeaker cone can be used to stimulate other physical objects, and finally the loudspeaker is destroyed to create a change in the sounding output.

\subsection{Lesley Flanigan}

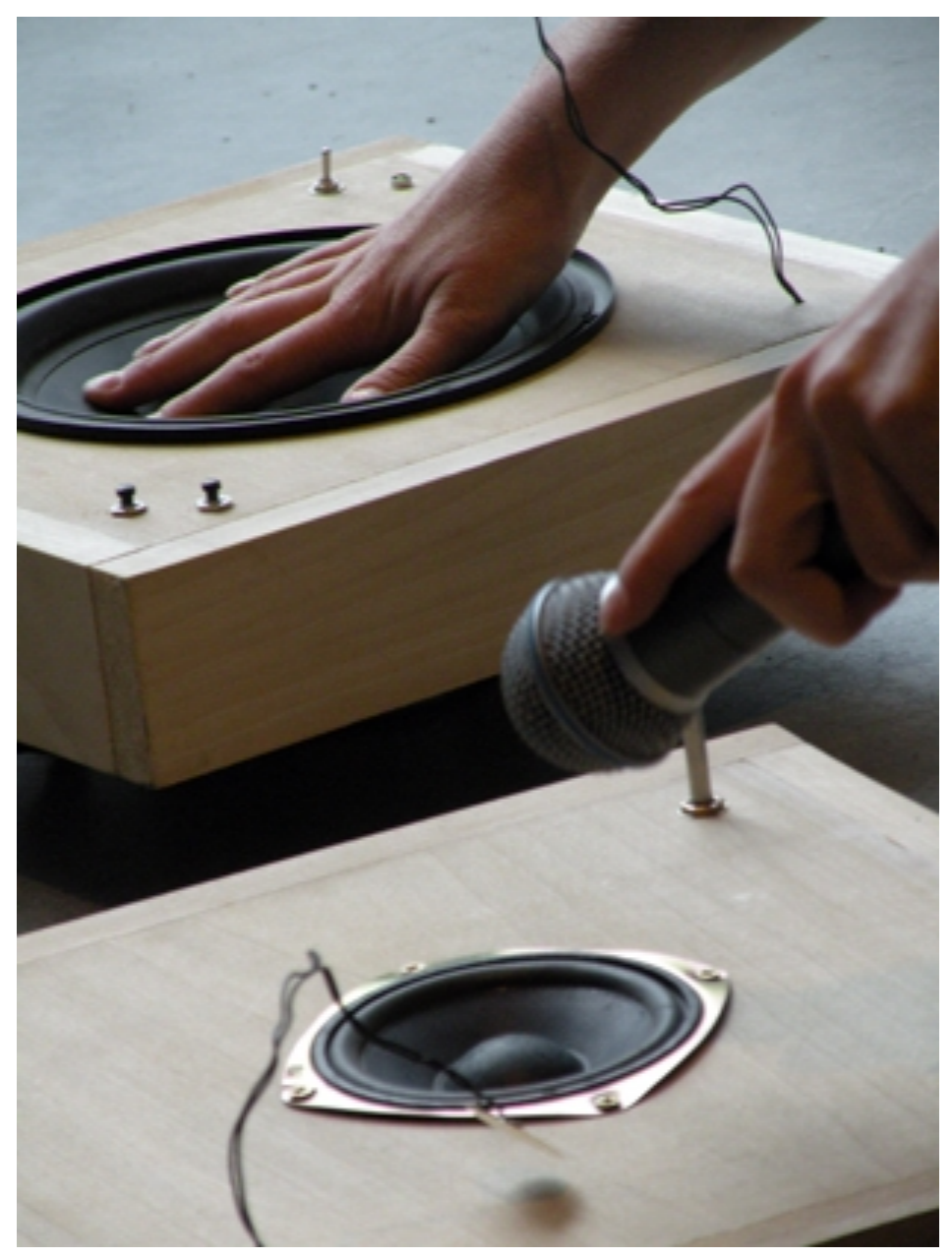

Figure 3. Lesley Flanigan's Live Performance 
Sound artist Lesley Flanigan not only builds her own devices, creating purpose built loudspeaker instruments, but also employs a 'hands on' approach to the loudspeaker in her work. In Flanigan's practice, the loudspeakers can be termed 'performative loudspeakers'. This is where the loudspeaker is pushed from a passive context, with the job of reproduction, to a performative context, where the role is integral to the sonic transformations of the work. This also means that, like an acoustic instrument, a performer is needed to physically interact with the object to extract a particular sound from it.

Direct contact with the loudspeaker cone is one of the parameters that allows Flanigan a degree of transformation upon the sound material, regulating movement of the cone to control feedback through the combination of contact and standard acoustic microphones. In a live performance at Bent Festival, ${ }^{10}$ Flanigan places her hands and contact microphones directly upon the cone to create manipulations and feedback. This material is in turn sent to PA loudspeakers, which are external to her setup. With the combination of harmonised noise and feedback, Flanigan enters her voice into the mix of sound, looping and harmonising with herself, creating an intensely intimate sound. This is mirrored with the sense of touch she incorporates into her work. The distinctive subtleties gained from the use of touch shows similarities of nuance gained from an acoustic instrument. Visually, the handcrafted wooden casing for the cones, along with switches and other controls, allude to a more traditional example of an individually crafted acoustic instrument such as a violin or guitar. The range of cones denote different uses, which can be seen within this performance where different loudspeaker instruments are called on to provide various tones in different frequency ranges. The microphone also becomes an instrument in this context, with Flanigan moving it back and forth in movements reminiscent of a string player's bow, controlling feedback with

${ }^{10}$ Flanigan, 2009 
distance from the surface of the loudspeaker. The microphone is placed on the cone, but this is not an obstruction, rather a technique to generate sound. As these loudspeakers are instruments, they are not limited to facing the audience. Flanigan lifts them up, cradling them and moving them with her on the stage. They are not static, but completely dynamic and integral to the performance and resulting sound.

\subsection{Gordon Monahan}

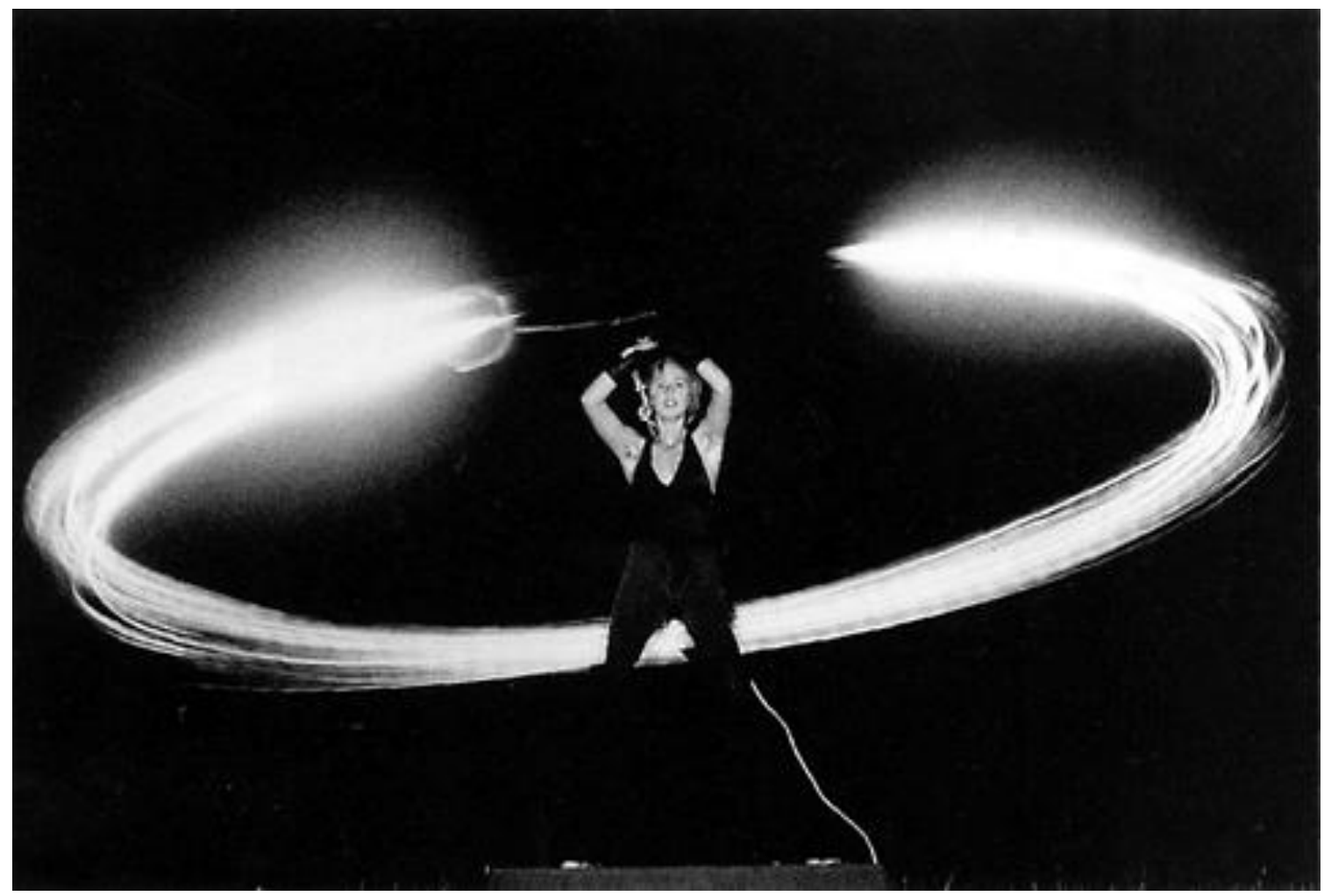

Figure 4. Gordon Monahan's Speaker Swinging (1982)

The performers in Gordon Monahan's work Speaker Swinging come into close contact with the loudspeaker without directly touching it. In this piece, multiple performers with one loudspeaker each are asked to swing a loudspeaker cone through the air by way of a long cord. While not in direct contact with the object, as in the case of Flanigan's work, the work relies completely on the composed spatial movement of the loudspeakers as an element that shapes the sound material of the piece. In this piece the performers are spread equally across a room. The sounds escaping the loudspeakers are various sine 
tones, unchanging in frequency and timbre. These do not change until the performers start to swing their loudspeakers, one at first, then the others one by one. As the cones reach the required velocity, the sine tones are perceived to fluctuate. Amplitude of the sonic material waxes and wanes periodically, according to the speed of the swing and the distance and direction of the cone relative to the listener. Because of the layout of the performers, some of the sine tones from the loudspeakers interact with each other where coupling and differences of frequencies cause beating, creating a chance situation in which the differing rates of swing allow for different timings of interaction. As the lights dim in the space, the movement of the loudspeakers are highlighted further with the inclusion of lights at the end of the cords, allowing the audience to follow the movement. This also serves to highlight the movement of the human body as we witness the strength and stamina needed to swing the cones. The exertion of the body in relation to the loudspeaker normally remains behind the scenes, carried out by the roadie or the removal team as they carry the gear necessary for the resulting performance. Monahan highlights the weight of the object and the inevitable transformation of the source sound as it is swung through space, juxtaposing the fragility of the object with the forcefulness of the movement it undertakes. 


\subsection{Ed Osborn}

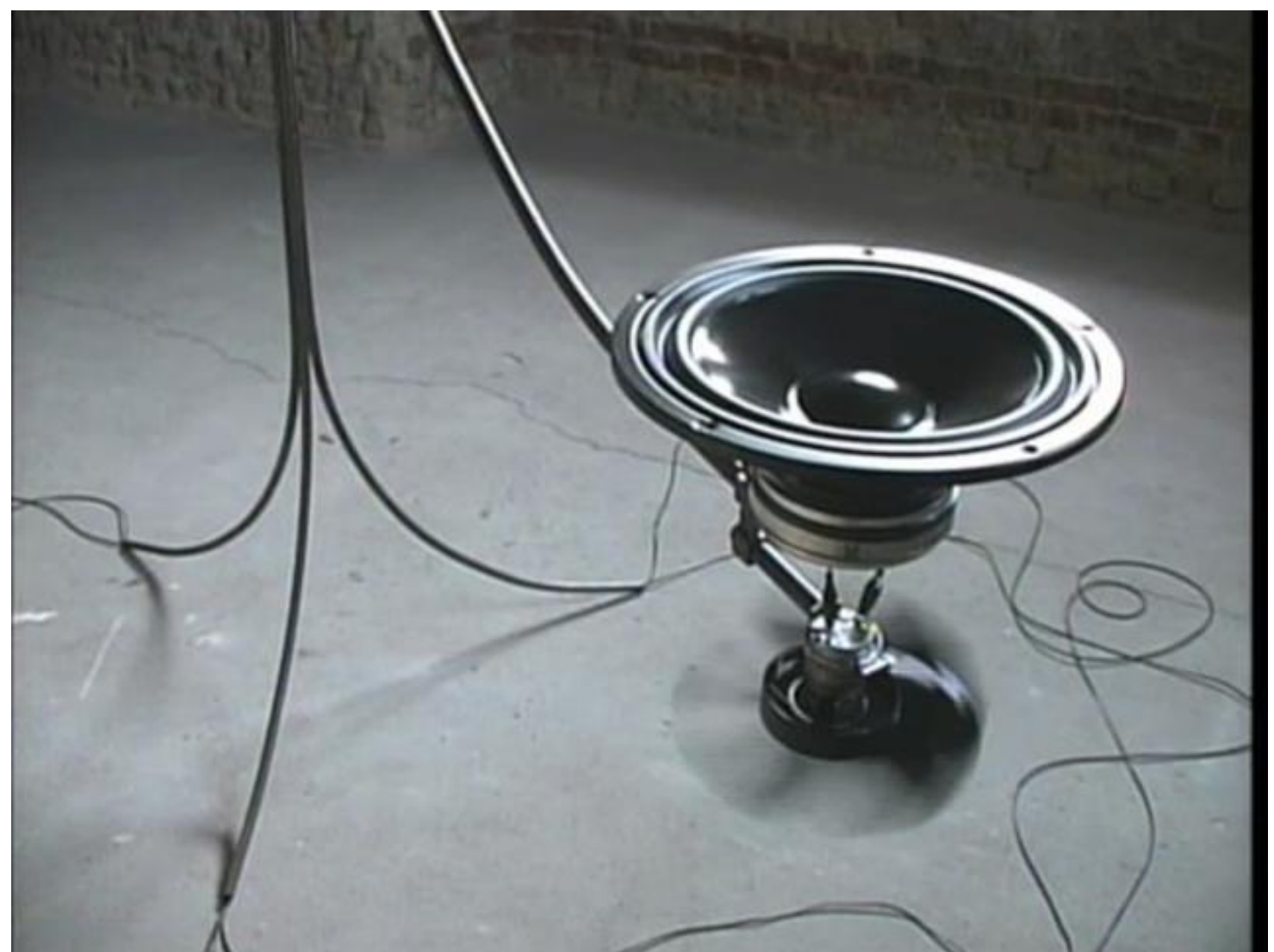

Figure 5. Ed Osborn's Flying Machines (2001)

In the case of Ed Osborn's sound installations and sculptures, a subtler showcase of movement is formed. The movement of the loudspeaker in these cases shapes the installation but is created as a consequence of the sculpture itself. In opposition to a more traditional model of sound installation, where the loudspeaker typically remains static, Osborn's work creates playful situations in which the perception of the sound material is directly shaped by the physical situation in which the loudspeaker is placed. In Flying Machines, two loudspeaker cones are precariously perched on each end of a construction, which is supported in the middle to resemble a seesaw. The balancing act is held in check by two fans, one underneath each cone. The varying rates and stopping and starting of these fans, cause the sculpture to waver on the edge of balance.

Complete and utter attention is brought to the object itself; will it fall? Three of these sculptures are in the room, creating an awkward ballet, full of tension as the audience 
watches the stumbling dancers. In combination with the sound material, 'vocalesque sounds that recall large creatures in a state of mild agitation', an 'acoustic mismatch' (Osborn, 2012a), is created. This works with the physical mismatch of flying loudspeakers propelled by fans, accentuating the physicality of the loudspeaker. Osborn's later work Swing Set (2002) also uses movement to draw attention to the object. This work is set up to resemble children's swings, with the five swing sets being placed in a circle. On top of each swing is 'a bare loudspeaker facing upwards' (Osborn, 2012b). What creates interest in this installation is not the noise and the static emitted by the loudspeaker, but its movement: for what purpose are the loudspeakers swinging? Unlike the movement of the loudspeaker in Speaker Swinging, the spatial movement in Swing Set is moderate, affecting the sound only minimally. The subtle questioning of purpose within Osborn's work may be just as effective in drawing attention to the object, as the explicit nature of Monahan's and Flanigan's work does.

\subsection{Jason Wright}

In my own work I have produced a number of studies that exploit the many physical characteristics of the loudspeaker, allowing me to modify the incoming signal on an external physical level. My work Tender Suffocation (2012), examines the effect of objects placed upon the loudspeaker to restrict movement of the cone whilst observing how this affects the audio output of the system. In the performative component of the piece, five loudspeaker cones are placed on the floor and are fed pre-composed sound material. This material, derived from piano and vibraphone samples, remains on a short loop that continues for the duration of the piece. As this loop plays, the performer proceeds to place small stones upon the cones. As this is gradually done over the course of 20 minutes, the weight of the stones starts to weigh the cone down, thus restricting its ability to fully reproduce the original sound material. As the stones accumulate upon the 
surface of the cone, they act not only as a weight, but also as a filter, distorting higher frequencies and muffling lower ones. As the sound loop is short and repetitive (Schaeffer's 'locked groove') ${ }^{11}$ the listener is able to assess the transformation of sound as the stones are placed upon the cones. By placing these objects on top of the loudspeakers and restricting the cones' movement the affects that are gained are: the dramatic decrease in amplitude and filtering of the original sound. Secondly, the sound of the stones themselves become audible as the movement of the cone causes the stones to shuffle and rub against one another, adding a layer of sound caused by the movement of the cones but external to the sound information the loudspeaker is attempting to produce.

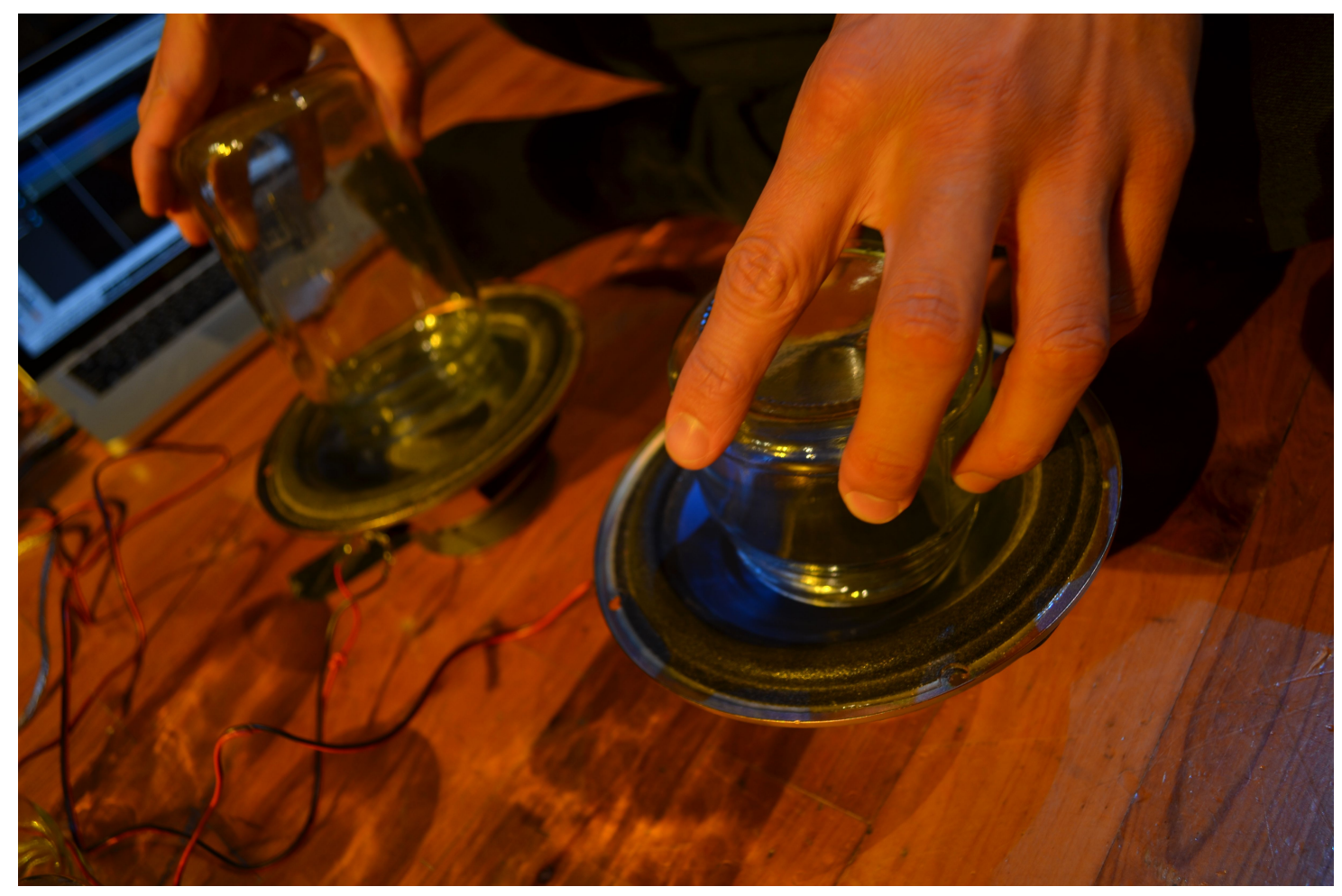

\section{Figure 6. Blue (2012)}

The loudspeakers in my work Blue are also examples of performative loudspeakers. The performance technique in this piece sees an object used to directly touch the loudspeaker, which in this case is a glass jar. In contrast to Tender Suffocation, where

\footnotetext{
${ }^{11}$ See Labelle (2006), pg. 27
} 
materials are placed upon the loudspeaker and left static, this piece sees me using objects in a far more active manner: physical interaction with the loudspeaker is undertaken as I use the jars to sculpt the sound emanating from the cones. The glass jars are used to press against the surface of the loudspeaker, and are manoeuvred and positioned to create a desired sound. The moving loudspeaker cone comes into contact with the glass jar, causing both objects in turn to vibrate and resonate. Through moving of the jar, I am able to activate resonances and harmonics that are separate to the material the loudspeaker is producing. By holding the jars upside down, I am also able to create sympathetic Helmholtz ${ }^{12}$ resonances in the jar. By holding it slightly above the surface of the loudspeaker and using subtle vertical movement I can control amplitude of the resonance. These changes in amplitude are external to the volume of the sound produced by the amplifier and loudspeaker once again creating change external to the mechanical system.

There are similarities here with Flanigan's loudspeaker performances, where the hand is used in direct contact with the loudspeaker cone. Where my work differs is that the sound of the jars vibrating is integral to the work, as are the stones in Tender Suffocation. In Flanigan's work, touching the loudspeaker produces different tones and levels of feedback, but the sound of the contact itself is not audible.

My work Glass also gives example of performative loudspeakers. A full explanation of this work can be found in Section B, Chapter 4.3 of this exegesis.

\subsection{Dick Raaymakers}

Raaymakers' sound sculpture Ideophone 1 (1970) provides an example in which the audible content of the work does not emanate from the loudspeaker, but rather is

\footnotetext{
${ }^{12}$ A Helmholtz resonator or Helmholtz oscillator is a container of gas (usually air) with an open hole (or neck or port). A volume of air in and near the open hole vibrates because of the 'springiness' of the air inside. A common example is an empty bottle. <http://www.phys.unsw.edu.au/jw/Helmholtz.html> (28 May 2012)
} 
produced by the contact of an object with the loudspeaker's surface. Raaymakers

explains the mechanics of his sculpture:

Ideophone 1 is an ensemble of 36 identically functioning speakers built into a chrome-plated metal case...the case can slowly swing and revolve on an axis within a 90-degree angle. A foot-long glass pipe about an inch in diameter is attached to each speaker cone at a right angle. Inside each pipe is a small steel ball... When the ball touches the fork, the speaker cone jolts forwards and forcefully propels it away. The ball rolls back inside the pipe, depending on the angle, and is kicked away again. The steeper the angle of the pipe, the faster the series of kicks from the speaker. If the case has reached the tabletop position and all the glass pipes are standing upright, the balls will be bouncing up and down at top speed inside them (Raaymakers, 2012a).

What results is a 'periodic series of impulses (which can be compared to a hailstorm)' (Raaymakers, 2012a). In this example the loudspeaker fabricates the sound of the system through activating its mechanism to create physical contact. Rather than producing external sound, the contact of the loudspeaker cone and the steel ball is the only aural component. In this installation the loudspeaker's function is reduced significantly and can be compared to a piston, pushing back and forth. What becomes important in this work is not the action carried out or the resulting sound, but that the particular object is framed and used in this way. Other aspects of physicality come to mind when viewing this work; the sense of fragility of the object is dispelled due to the implied robustness from the repeated contact with the steel balls. Although this may indeed be damaging to the loudspeakers over the course of the installation, what is shown is the ability of the object to engage with a level of physicality with which it is not usually attributed. 


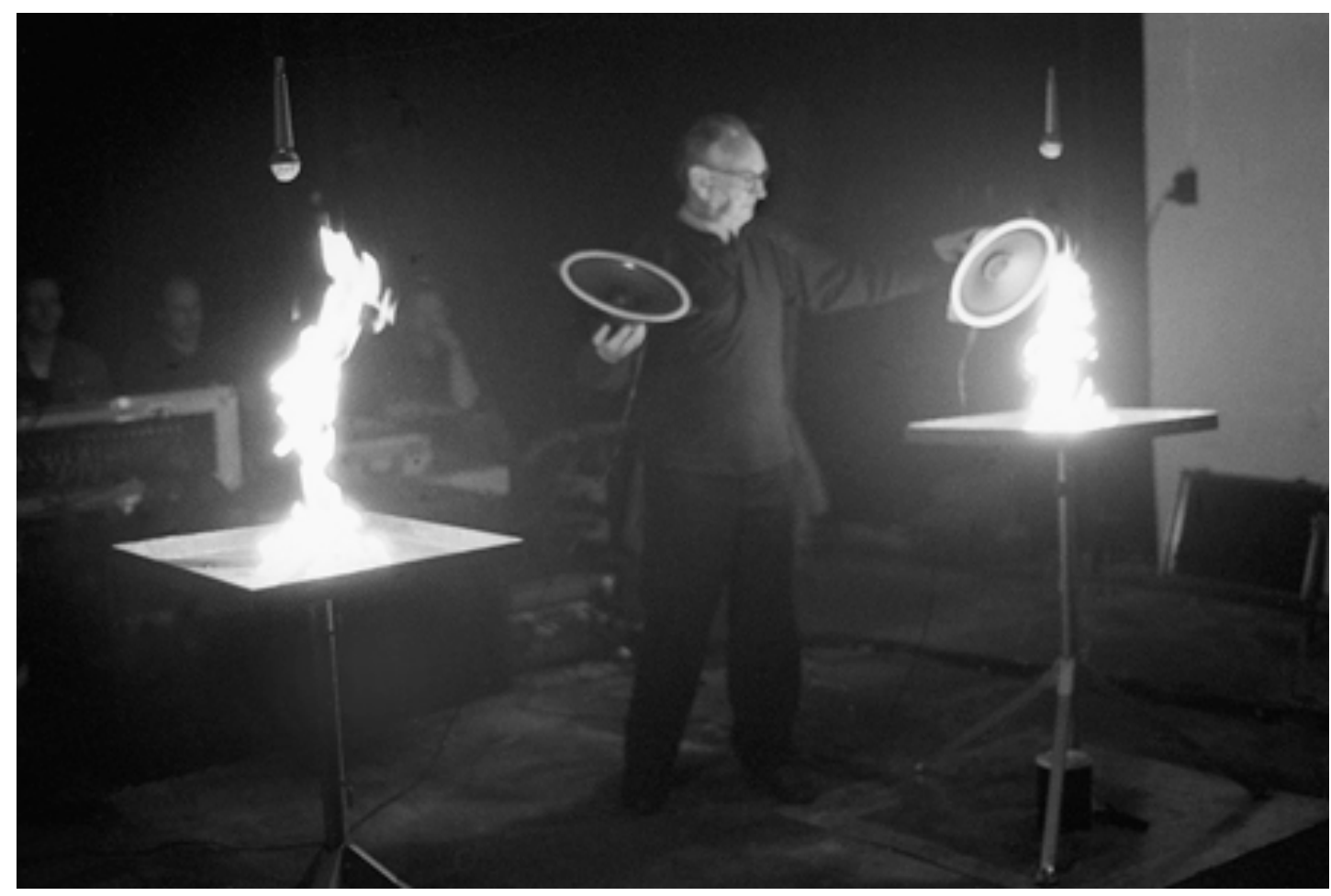

Figure 7. Dick Raaymakers' Intona (1992)

Intona, discussed earlier, provides an example of destruction of the loudspeaker. For the final act of the work, Raaymakers places two bare loudspeaker cones within naked flames upon a stand. Having destroyed several microphones, the next step is to snuff out the objects that up until then, had produced the sounds of the microphones, '(venting) their protests against the way they have been played' (Raaymakers, 2012b). As they are burning, the sound the loudspeakers are producing slowly dies away until only the sound of the flames is audible. This external element causes the mechanism of the object to slowly break down, until its original capabilities are extinguished.

As this discussion has shown, perceptual weighting of the aural component of the loudspeaker shifts when it is placed within a performative context. The sound emanating from the loudspeaker was once the only point of focus, now the object itself draws attention. These works need to be considered holistically; the technology and how it is used within the performance or installation system is as important as the aural outcome. Unlike many acoustic instruments, which have a rich history of performance 
practice and tradition, the loudspeaker in performative and tactile dispositions is relatively new and unexplored. In assessing this new performance situation, new considerations need to be taken into account to bring the loudspeaker closer in nature to a traditional instrument. What is also shown in this chapter is the ability of the loudspeaker to create perceptual and aural changes in sound via the modification and playing of its mechanism and body. Engaging the loudspeaker in a tactile sense leads to the transformation of a pre-existing sound object and the possibility of creating entirely new sound objects.

\section{Visual}

A single speaker, at least is something to stare at (Eisenberg, 53).

Engaging the loudspeaker in a visual sense may seem counter-intuitive. When surveying its usage in both performative and public contexts there is an inclination to conceal the object from view so as to not break the illusion of transparency.

Loudspeakers blended into the wall at a café or hidden by dimming the lights at a concert are all attempts to eclipse the object and hide any trace of technological mediation. Transmission Audio provides us with the 'In-Wall' design for the home, where loudspeakers are made to 'disappear' as they are placed 'directly into a wall in order to make them truly invisible' (Transmission Audio, 2010). 


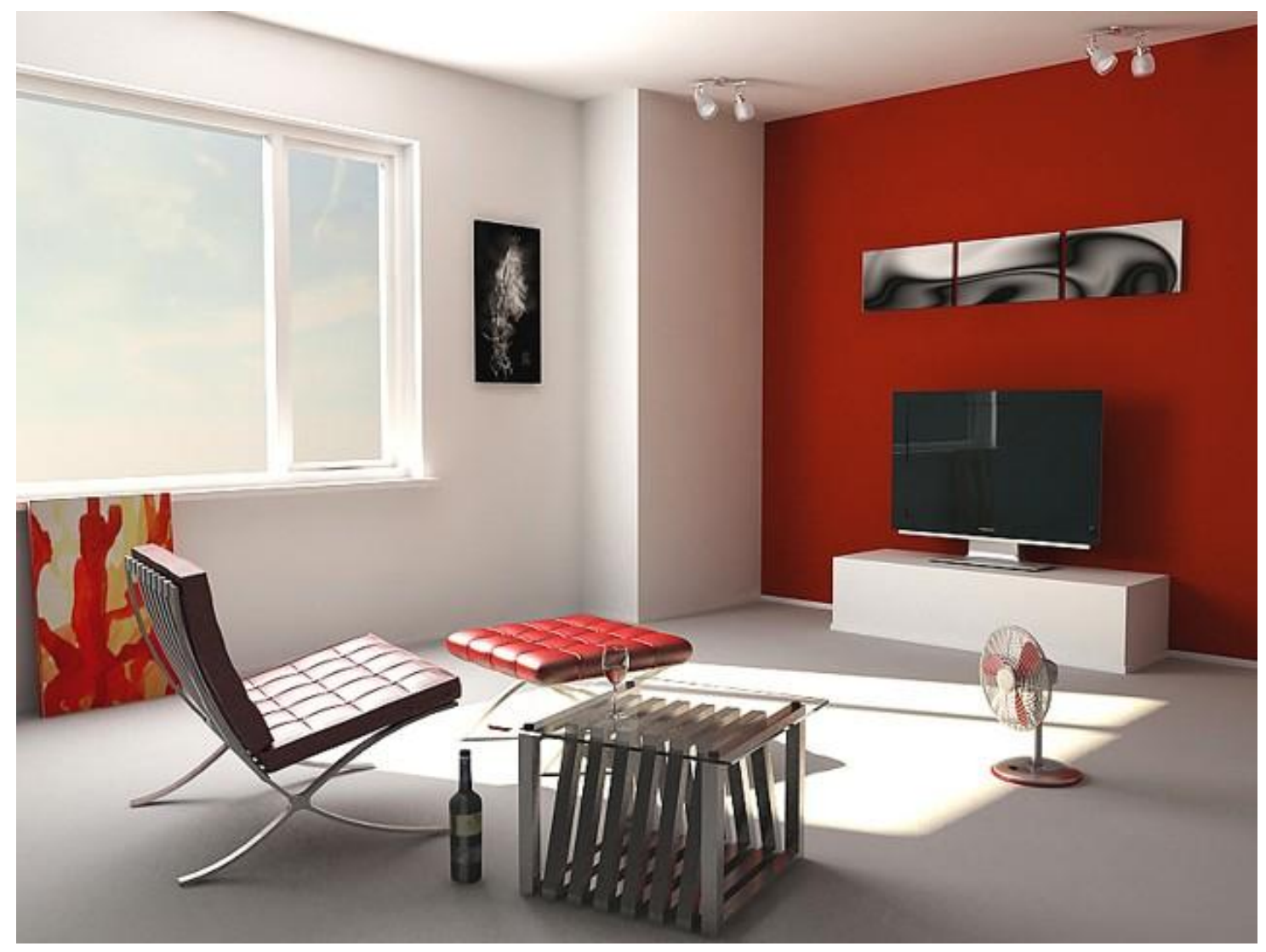

Figure 8. Transmission Audio's 'In-wall design. Can you see the speakers...?'

One of the points I address in this chapter is the fact that the attempts made to conceal the loudspeaker from view are fruitless. Through the saturation of technology within most facets of everyday life, loudspeaker listening has come to be a complete part of our music reception cognition. So much so that when auditioning music, with either electronic or acoustic source material, we take into account aural and visual clues that lead us to infer the loudspeaker's presence regardless of whether or not we see it. Philip Brey says this about our embodied relationships:

Our cognition of embodied artifacts themselves is puzzling. We often do not seem to be consciously aware of them, since after an initial habituation period, they fade into the background of our awareness. Yet, the skillful use we make of them reveals that we possess detailed information about them, for instance about their location in space (Brey, 1).

What I explore here is how we engage the object, the loudspeaker, visually. The way we reconcile the split between the audio-visual live performance and the acousmatic 
listening situation, brings into question loudspeaker listening and whether the loudspeaker has a visual component that acts upon our perception of music. There is evidence to suggest that a visual component detracts from our reception of music. David Hendy looks at this in his article Listening in the Dark, where he addresses 'cognitive research that shows people's mental imaging to be richest when they are not bombarded by 'interference' from externally produced images' (Hendy, 223). The implementation of this idea can be seen in acousmatic concerts where lights are dimmed to remove any visual stimulus, allowing full concentration on the sound and music. However, when attention is brought back to the loudspeaker, what are the aspects of this object that inform our perception of sound? Do aspects of its design and presence inform listening? Do these aspects correlate or not with the sounding properties of the music, or are these aspects mutually exclusive?

An advertisement from a 1994 issue of Gramophone Magazine contains an advertisement for Monitor Audio LTD with the slogan for the loudspeaker stating; 'To listen. To see. To feel' (Gramophone, 1994). This emphasis on 'To see' showcases the loudspeaker as an object that is there to provide something visually for the consumer and should be taken into account as something equally important as the primary function it provides. Mitchell Whitelaw says of the loudspeaker: 'Its surface is never artless. It's a produced face, an engineered and designed face... It makes a visual display of its engineering... Sound Production and image production blend' (Whitelaw, 1).

\subsection{The Loudspeaker as Visual Artifact}

In some circumstances the loudspeaker becomes an artifact of a completely visual nature. The placements of 'retro' loudspeakers in a shop window are not there to reproduce music, but to articulate a 'vibe' and an element of personal aesthetic. This 
negates the function of the object but allows the 'user' (in this case as opposed to the 'listener') to extract additional information from it. Peter Verbeek explains: 'it [the product] has meaning or sign-value: human beings are drawn to particular product styles and not to others, and use a product to express the lifestyle to which they (want to) belong' (Verbeek, 204). But in this case do we infer sound attributed by the function of the object, or do we accept it completely as a visual artifact? The framing of the object in this case is all-important. Behind the shop window, with no direct path for clear sound output, one may deduce, regardless of whether they can hear sound or not, that the object in question is not being used for its intended function. By contrast, a loudspeaker placed next to a set of turntables becomes framed in a functional context. Although still retaining sign value, the affordance of the object shifts slightly, moving into territory where transparency again becomes important. In the case of the loudspeaker in the shop window, the object becomes extended and is turned into a sign, representative of socio-cultural characteristics of the user and surroundings: 'Signs can be treated as means for an ends: an automobile is a means not only for transportation but also for expressing ones status; a coffee pot is a means not only for producing coffee but for exhibiting ones taste' (Verbeek, 208). As mentioned above, a loudspeaker, which is made to match the walls in a café drops significantly out of our visual reference; the listener's cognitive priority rests on its functional value. Combining both functional and sign value in more equal proportions is the home stereo system where the element of social status, as well as the performance of the object, plays a part in the choosing of the artifact. Again Verbeek provides evidence for this:

Objects can acquire their meanings for people first of all from their intrinsic aesthetic qualities. That is true, naturally, for artworks, but also for useful objects in general to the extent that people find their exteriors pleasing or that they express a particular lifestyle with which people want to be associated (Verbeek, 223). 


\subsection{Sound Art}

Through reframing of the object in an artistic context, can the visual component be accentuated and made the basis of a work? Through examples in this chapter I will show the loudspeaker to be a visually complex object, where perception of its visual components is informed by knowledge of its function, and the expectation thereof. In these works the visual and audible dimensions are juxtaposed, creating contention in an attempt to decipher and reconcile the dimensions of the works.

Two artists' works, again considered alongside my own work, are detailed below. Within these works are examples of loudspeaker installations with no sounding content, exploring the visual element as primary over the functional and audible elements of the object. This is contrasted with examples of my own work where I will show how examining certain pieces acousmatically can be detrimental to fully understanding the work. In these cases the visual component provides significant information that links the audible content with the underlying concept.

\subsection{Tristan Perich}

Sound artist Tristan Perich draws the loudspeaker into territory where meaningful visual stimulus is provided and paired with a lack of sounding content. This creates tension for the audience through a definite role reversal and shift of expectation of the object. Perich's installation Breathing Portraits (2005-2010) represents breathing patterns of selected subjects, recorded on various nights during sleep. These breathing patterns are made visible as the movements of various loudspeaker cones are controlled by precise oscillation of electrical current. Perich is able to physically mimic the breathing patterns of the individuals, creating incremental contractions and expansions of the speaker diaphragm to represent the chest of the human body. Situated in a gallery space, the only sounds present are the hum of activity outside the gallery. The loudspeaker in this 
instance is moving too slow and un-periodically to create any sound, existing rather in a state of potentialities, where the movement created by the 'sub-audible waveforms' (Perich, 2010) can only be seen upon close inspection. In juxtaposition, the loudspeaker's opposite state, that of constant movement to generate sound, also requires close inspection to observe the movement of the cone. At stages above $25 \mathrm{~Hz}$, the precise movement of the cone becomes difficult to discern.

In this installation the focus is brought to the body of the object, the physical mechanism and by way of content, the human body. The associations made between the loudspeaker and the human body are apparent with each loudspeaker assuming the name of the human it represents. We gain a sense of the movement of the subject's bodily movements through the loudspeaker as it is used to evoke image and display movement. Whilst not a direct sensory result of the installation, Portraits invokes and implies sound by playing with the expectations of the object used. As I mentioned above, the loudspeakers sit with the potential for sound to be generated, thus as the loudspeakers are placed before us, our senses will be heightened towards sound; the loudspeaker by way of its inherent functionality creates expectation. The subject of Perich's installation points us towards the sound of breathing. We are inherently familiar with the sound of breathing and may imaginatively overlay this sound upon the installation ourselves. In some cases, where the audience member's breathing becomes regulated according to the particular cone they are watching, their breathing may become audible, thus becoming sonic content within the work. Through the visual focus of the installation, attention is brought to the physical body of the loudspeaker. Through the element of breath the subject of our own corporeality is addressed. 
McKinnon says this about Portraits:

The loudspeaker cone ceases to be the reproductive source of a spectral presence and becomes an uncanny thing, a hyper-object, the affective power of which derives from its ontological distribution across visible presence and audible absence (McKinnon, 2011).

The loudspeakers in Portraits become what they are, not because of the concept portrayed, but because of the shift that takes place in the technology that carries out this concept. This transition to becoming a 'hyper-object' does not happen to the loudspeaker in the living room, although it may be devoid of sound, yet in Perich's work the object is perceptually contorted until the function of the original object is mislaid.

\subsection{Sean Kerr}

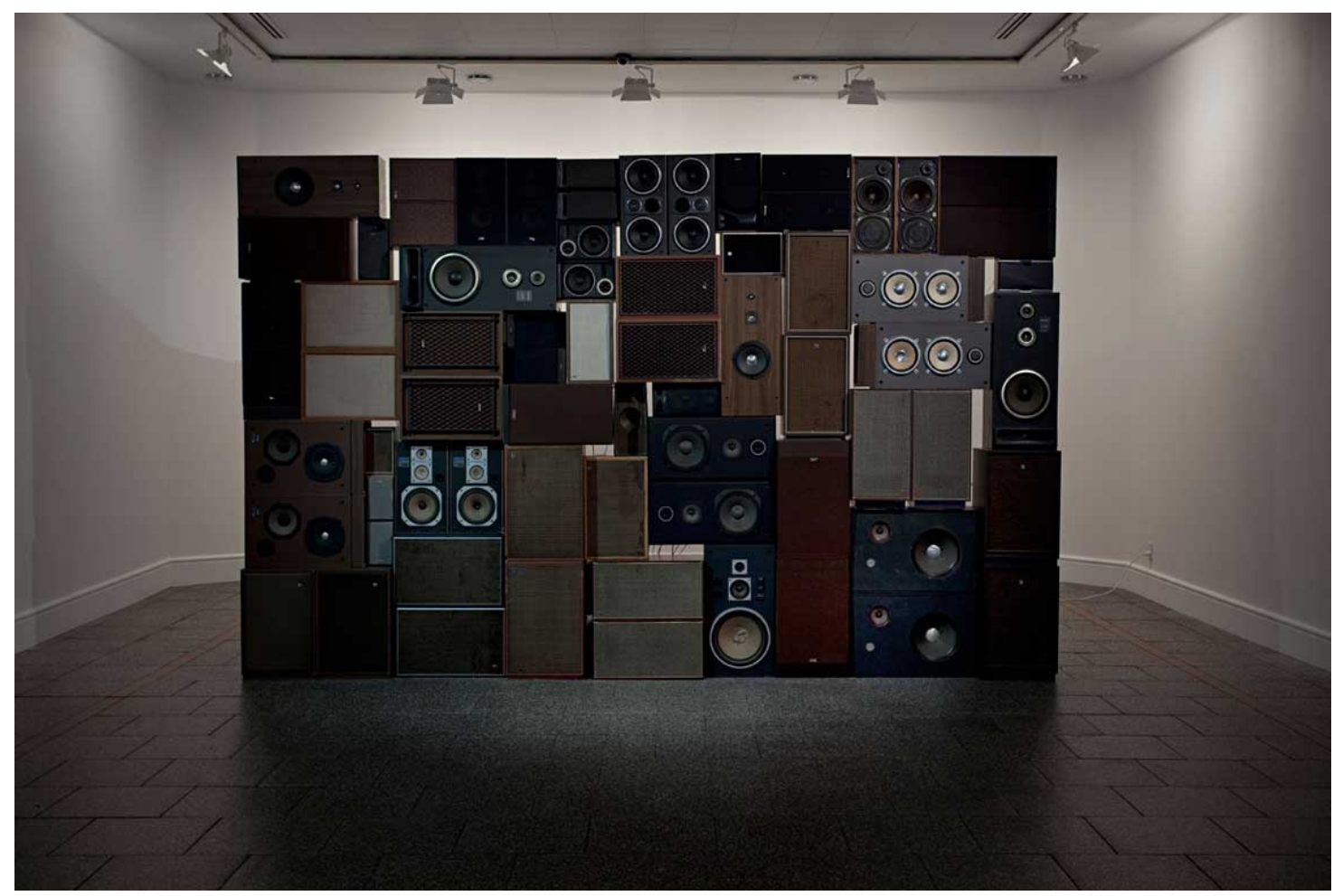

Figure 9. Sean Kerr's Stacker (2001)

Sean Kerr's installation Stacker makes a move toward accentuating and bringing to focus the visual qualities of the loudspeaker. Through the absence of sound, Kerr's 
work allows for a perceptual purging. As the aural component has given way to the visual in Stacker, the installation comes closer to being an image of a loudspeaker rather than a three-dimensional material object. Stacker consists of a stack of sixty-eight loudspeakers. Mostly consisting of matching pairs, these loudspeakers all range in size, colour, design and age. The loudspeakers are piled in a single layered square and resemble a wall. Placed within a stark gallery space, Stacker's presence commands attention.

This work is primarily visual; sound is implied through association of function with the object, as in Portraits, but the structure itself remains silent. Meticulously placed in pairs, discarded hi-fi loudspeakers ominously look out into the space. In this work we access something unique: Kerr's contextualisation of the loudspeaker within the gallery space allows the object to exist without the pressures of generating sound. The affordances gained in the context of the sound installation reduce any assumptions based on the objects function, as Kerr's reframing necessitates a reduced view of the object. Without the expectation of sound, the nature of the object and our relationship with it also shifts. We are no longer in control of it, and cannot endlessly extract audible content from it at our will. Stacker does not represent sound; rather it opens our evaluation of the object into territory where we can consider our interactions with it holistically. In framing the question 'what does the loudspeaker mean to me?' Stacker draws together elements of nostalgia, sound, aesthetics and design. This provides the possibility for engagement with the object in less concrete terms, such as visual appeal, instead of regarding it in more technical and esoteric terms. What we are left with is Kerr's insistence that the loudspeaker be taken seriously as a visually complex object, an object that the viewer can extract from on levels beyond its primary function. 


\subsection{Jason Wright}

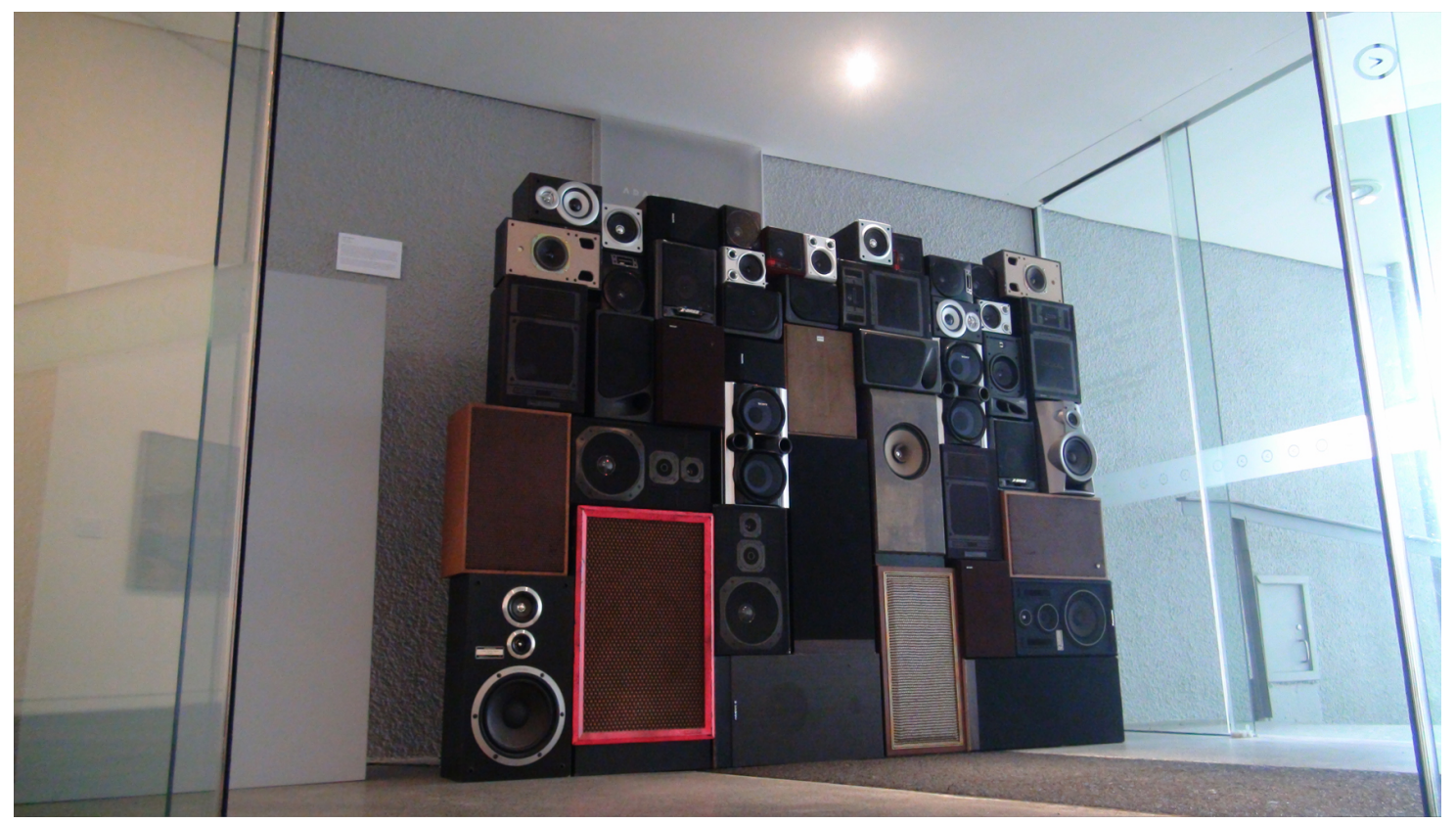

\section{Figure 10. Jukebox}

Following a similar vein to Stacker is my installation Jukebox. Although visually similar, this work differs in that it contains an audible element. Jukebox tries to encompass the idea of the loudspeaker as an un-solidified form, joint in purpose, but with a range of visual realisations (this resembles the type/ token distinction as mentioned in Chapter Two). Playing on the perception of sound informed by the visual, Jukebox encourages the listener not only to hear, but to see the difference in sound as the audience comes to reconcile the visual information with the audible information provided. This allows the audience member to be more aware of the physical attributes of the loudspeaker that inform the music, thus perceiving sound differently according to visual stimuli.

As it stands, all of my practical work for this project is audio-visual, not only in documentation but also in actuality. The visual component along with the audible element forms a composite. It is necessary to consider both of these elements in tandem to fully make sense of the work. Considering the sonic element of the works exclusively 
would see the work suffer conceptually and perceptually, as there would be insufficient sensory information to combine to create the work. This has been coined the "perceptual "lump"' and is gained from the 'global "impact" of the event' (Chion, Brewster, 71). Why I term these works 'audio-visual' is to stress the importance of the visual focus of the loudspeaker. Secondary to this is the way in which the loudspeaker is used: the extended techniques require visual surveying to fully grasp a detailed sense of what is transpiring. Auditioning my work Blue acousmatically would neglect the purpose of the piece, which is to show the loudspeaker as an instrument in an ensemble. At this point, one could talk about musical validity of the work, examining intrinsic factors of the music. However, this analytical approach would leave the work poorly assessed as the intended focus lies in the physical manipulations undertaken. Likewise, my piece Tender Suffocation has a performative aspect and can be considered a process piece, where the visual and audible elements support and compliment each other. Here I am setting up a distinction between a sound-based art form and music. Music may be auditioned and understood as detached from any visual stimuli, whilst these soundbased artworks require the comprehension of both facets simultaneously.

A sense of spectacle is often tied to the visual. In the next chapter I will address largescale loudspeaker installations that are examples of the spectacle; where fetishism emerges in ways that question the use of the object, the concept of the piece and the resulting effect of the work. The discussion of the visual will continue in this next chapter as it is integral to the particular discourse and marks a large component of the works to be addressed. Providing context for this I will look at the loudspeaker as an artifact of fetish and commodity. 


\section{Large Scale Works}

...the power of the machine is harnessed to a corrosive ideology which constructs existence as the struggle for self gratification in the form of commodities (Shaw, 21).

\subsection{Technological Fetishism}

According to Verbeek, many artifacts and personal belongings not only have functional value, but also they retain a sense of signification as a sign. This can be a sign of social status, or be representative of lifestyle, personal choice and individuality. Herbert Marcuse gives an insight into this particular phenomenon in One Dimensional Man (1964), where he states:

The people recognise themselves in their commodities; they find their soul in their automobile, hi-fi set, split level home, kitchen equipment. The very mechanism which ties the individual to his society has changed, and social control is anchored in the new needs which it has produced (Marcuse, 9).

The projection of the self onto one's possessions becomes significant. Objects become fused with their owners, having profound socioeconomic implications, which Marcuse makes clear. The particular loudspeaker, along with musical choice of the owner, become an amalgamated sign, providing a means to project a sense of 'individuality' out into space: 'a territorial projection' (Whitelaw, 2001). What results is your sound through your loudspeakers:

One aspect that has moved more and more into the foreground over the years, however, is the emphasis on the visual properties of objects; the aesthetics of design has grown increasingly concerned with the visual appearances of things. Under pressure of the increasing demands of marketing requirements, many companies have focused less on improving the quality of the object itself and more on its external appearance. This visualism has culminated in the postmodern obsession with signs and meanings. When products are turned into objects whose primary purpose is to refer to lifestyles, the emphasis is fully on visual qualities (Verbeek, 209-10). 
Here Verbeek's focus is purely visual, but as evident in Chion's modes of listening (Chion, 1994), sonic elements are no less susceptible to falling into categories of signs and their meanings. The combination of both the technology and the sound combine to create an informative view of the individual.

The separation of the object from its function and its transformation into commodity, has led to confusion about the nature of the loudspeaker, creating tensions surrounding its purpose. What ensues is an example of what William Leiss terms 'Technological Hyperbole', an 'unwarranted exaggeration of the anticipated social effects of new technologies. It is often matched by 'technological fetishism, which makes too much of new technologies, as if their features could magically transform our lives' (Leiss, 5). The iPhone and iPad are excellent examples of this phenomenon. This also bears direct relationship to how we perceive the musical object itself and the plethora of technologies surrounding musical culture. It would seem the shift has moved from where technology serves the music, to the complete reverse: the three-minute pop song itself the product of a technological limitation. Here it could be argued that music actuates the technology, 'today's generations divide over how they listen to music, not what kind of music they enjoy' (Coleman, 2004). At one extreme, in some circles it is the elements surrounding music that are the chief focus. Audiophiles and technophiles make judgements concerning intrinsic and extrinsic levels of the technology, while the music auditioned is diminished. Music thus becomes Muzak, an object whose role is to activate the loudspeaker: 'Some audiophiles worship their audio equipment more than they love music' (Cited in Perlman, 790).

The extreme poles of this fetishism lie in the area of commodity where the technology exceeds physical accessibility and mainstream economic attainability. Whilst allowing the fetishisation of sound, the object in question can also transcend its inherent 
functionality and come to represent something else entirely. There is no fine line to this distinction, as a condition of the object to remain 'a loudspeaker' is that it has the mechanical potential to produce sound. This means that the object will always remain inherently functional. However, it is how the object is framed that allows for evaluation of what the object stands for. In these following examples the object moves between remaining functional and providing a spectacle. Guy Debord comments on the potential difficulty of distinguishing the two facets:

The fetishism of the commodity - the domination of society by "intangible as well as tangible things" - attains its ultimate fulfillment in the spectacle, where the real world is replaced by a selection of images which are projected above it, yet which at the same time succeed in making themselves regarded as the epitome of reality (Debord, 1994/ 1967).

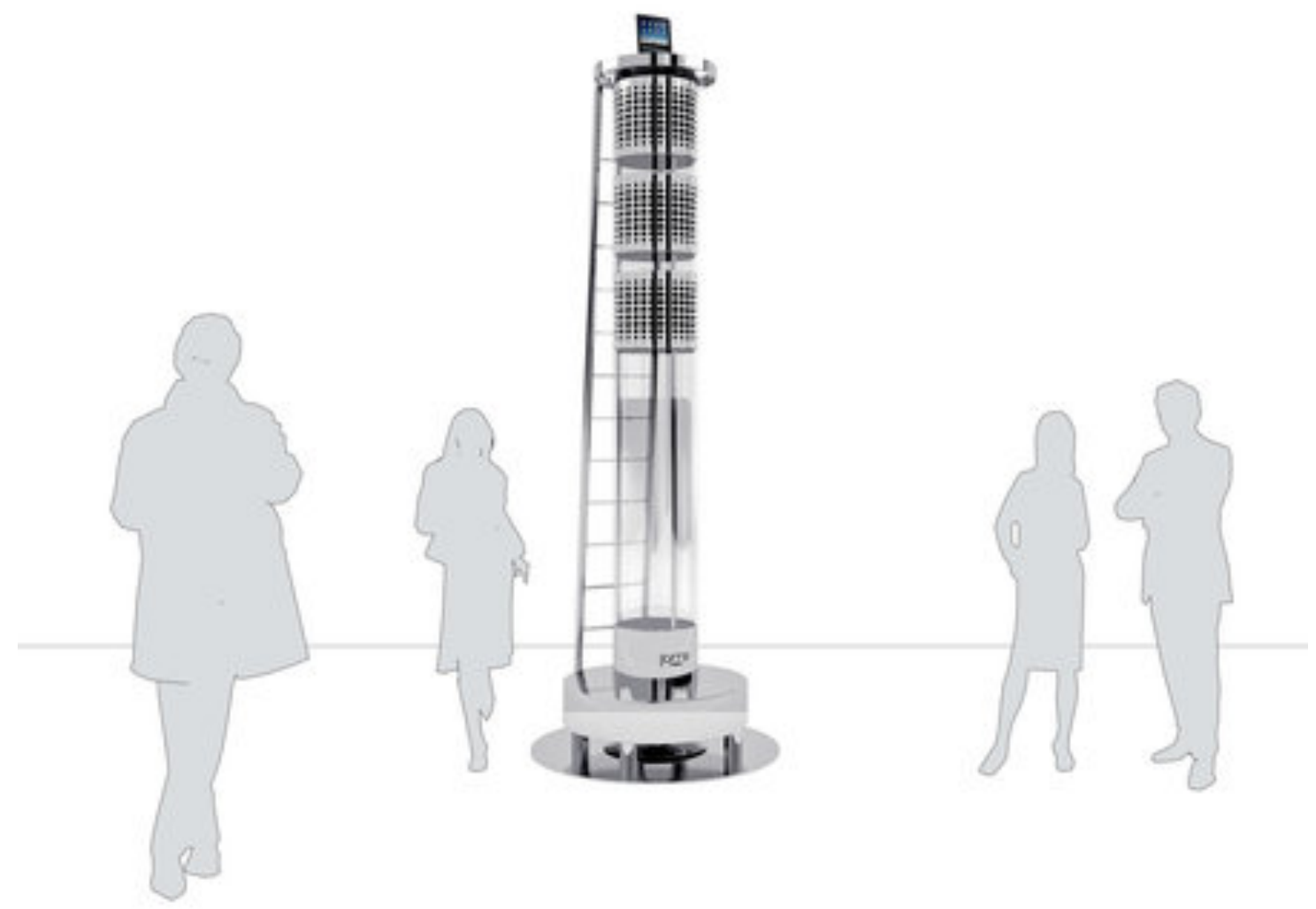

Figure 11. Jean Michel Jarres' Aero Dream One 
Jean Michel Jarre's custom-built loudspeaker system, the Aero Dream One $e^{13}$, stands at 3.4 metres tall and requires the use of a ladder to place an iPad or iPod within the 'dock'. Although looking impressive within the showroom, Aero Dream One would require a vast space to house the unit, requiring an uncannily high ceiling or the acquisition of a mansion-like residence. Weighing 395 kilograms, the practicality of the object is called into question as the promise of 'optimal performance' (Jarre, 2011) and fidelity may be overshadowed by limitations posed by both its physical size and weight. Definitively unique in design, the towering product takes on an elitist component as it operates on a made-to-order only regime, taking 6-months for the system to be complete.

Similarly, with not only its imposing size being a factor, sitting at over ten metres wide, the cost of Transmission Audio's Ultimate loudspeaker system ${ }^{14}$ may be a potential drawback for would-be audiophiles. Costing one million dollars (US) per channel (two million dollars for a stereo pair), Ultimate is 'probably the finest speaker system ever built' (Transmission Audio, 2010). This expansive system takes 9 months to make, from order to delivery and includes an impressive and expansive list of components ${ }^{15}$ weighing 3650 kilograms. As denoted by its function, Ultimate, like Aero, provides precision and fidelity in terms of the sonic experience. As an artifact these two systems represent a love for sound, but maybe more importantly a love for technology. Alongside this is the need to showcase ones identity through acquisition and display of the commodity.

\footnotetext{
${ }^{13}$ See Jarre, 2011

${ }^{14}$ See Transmission Audio, 2010b

${ }^{15}$ It is outside the scope of this exegesis to detail these components here. For a full description of these components, see Transmission Audio, 2010b
} 


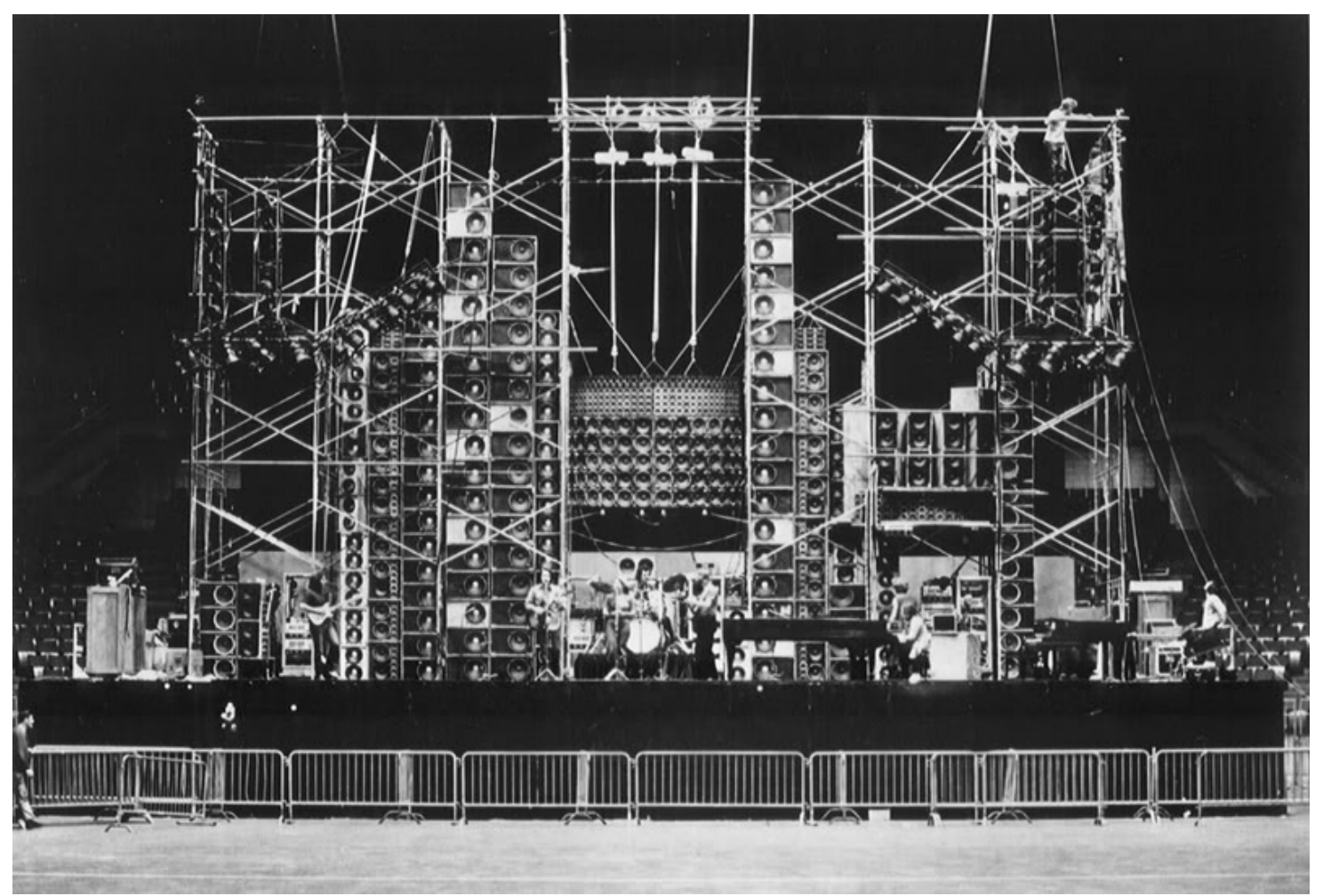

Figure 12. The Grateful Dead's 'Wall of Sound'

Another example of creating identity through the object can be found in the 'Wall of Sound, ${ }^{16}$ created by the sound engineers of the band The Grateful Dead. The 'Wall of Sound' is a monolith of loudspeakers where huge towers make up the six individual PA systems each belonging to the individual instruments onstage. This element of spectacle was integral to the stadium rock era of the 1970s and The Grateful Dead endeavoured to have the biggest and loudest sound-system, creating an experience for the audience that would not be surpassed, visually or aurally. The 'Wall of Sound' became an emblem for the band, representative of both the music and the lifestyle of the era. This example shows a fascination with the sonic experience, creating a sound-system that would surpass all others in amplitude, fidelity and spectacle.

This veneration of the loudspeaker is related to Alfred Haddon's $(1921,70)$ definition of the fetish: "namely, the worship of 'an intangible power or spirit incorporated in

${ }^{16}$ See 'Wall of Sound', 2012 
some visible form"' (cited in Ellen, 215). Through the loudspeaker, sound is worshipped. Following this argument, the object surpasses the sound it can produce and becomes the cynosure of the listener. The deconstruction of the relationships between loudspeaker, music and listener creates a definite grey area where roles are no longer distinct: 'the turning of ideas into objects inevitably involves an element of separation, de-contextualisation or externalisation (Taussig 1980: 460, following Whitehead)' (Ellen, 222).

\subsection{Sound Art}

In the work of John Wynne and Benoit Maubrey the breakdown just described is accentuated and brought to the forefront of the work. Aspects of fetishism, hoarding and also a sense of ritual become apparent. Masses of loudspeakers are placed in public spaces and galleries forcing the audience to engage with the object and meta-object through the sheer overwhelming presence of the objects. Viewed as an instance of the technologically sublime, the veneration of the object overshadows the sound it was created to produce: 'The ideal is no longer live music, but some technologic platonic form' (Eisenberg, 90). Although meeting greater conceptual demands of the works, the use of the loudspeaker in such cases borders on excess. In these pieces, loudspeakers are arranged to replicate physical edifices and create structures big enough to house several people. These composite objects made of loudspeakers transcend the individual object. While they still retain recognition of the individual parts, what is created are metaobjects. These allow differing perceptual ratios of sound and sight depending on the perspective with which we view and hear the work. The loudspeaker again becomes a 'hyper-object' where the 'ontological distribution' (McKinnon, 2011) of the visual and audible components is unstable due to the radical framing of the object. In contrast to everyday use, the loudspeaker here becomes completely disembodied. Attention is 
drawn to it and it becomes the direct 'object of perception' (Brey, 3) with no possibility of withdrawal from our consciousness.

In these works sonic material is minimal and in some cases recurrent over multiple installations, as is the case with Maubrey's pieces. Large-scale installations provide the opportunity to engage with the work on many levels: we experience the work with our whole bodies, not only our ears and our eyes but we are also required to physically move around the work to engage with it sculpturally.

What will be looked at here is the consequence of the structure and the resulting spectacle and what this means in the context of a sound installation, asking the question: when the object used makes its prominence as a commodity and infiltrates this sound art practice, what kind of implications does it have for the work? Although touching on the sonic component of the works, this will not be a focus for this particular section. I will address how the extreme nature of some of these works show the aural component to be secondary and how it may be overlooked in light of the visual component. While the conceptual component of these works remains unaffected, or even accentuated, it is interesting to note how the relationship between the visual, the aural and indeed the audience, changes due to the scale of these works.

\subsection{John Wynne}

John Wynne's loudspeaker installations venerate the object. Containing no fewer than two-hundred loudspeakers within each piece, Wynne's collection showcases a collector's mentality. Not just any loudspeaker will do, hi-fi loudspeakers are chosen specifically. When surveying these pieces it becomes evident that size, shape, colour and quality are definite considerations in the intrinsic elements of the composition. The visual component of the work relies on a specific aesthetic, one that takes into account uniformity and control. For this reason these pieces are suggestive of social order and 
hierarchy. Wynne mentions in an interview with the $\mathrm{BBC}$ about his piece 230 Unwanted Speakers (Walnut Grained Vinyl Veneered Particleboard Construction) (2006); 'When I started gathering speakers I noticed that they each had a personality... and when I started grouping them in large numbers it took on a kind of social metaphor' (Wynne, 06). Dignified in nature, the detail and order with which the loudspeakers are placed, becomes reminiscent of a population of objects, all interconnected. Wynne's Fallender ton für 207 lautsprecher boxen [Falling tone for 207 speakers] (2004) evokes the metaphor of the crowd. Each loudspeaker is placed on the floor facing into a central point of focus, the principal loudspeaker is hung upon the opposite wall. Wynne said this about the audience response: 'Consequently, some visitors saw it as a concert hall scenario or a choir, others as a cityscape, still others as a political rally' (Wynne, 2004). While creating a societal metaphor, 230 Unwanted Speakers shows Wynne to be an accomplished collector. This aesthetic of uniformity within Wynne's works relates a certain ideal, an idée fix. The condition of the loudspeaker is pristine. The fragility of the setup and the care with which the loudspeakers are placed is evident. Placed in a circumference in eight distinct groups the loudspeakers in this work are all largely similar in colour: a particular shade of brown. These are placed around a central point of a second group of loudspeakers, which are spread in different directions gazing back out at the congregation. These are white. Matching colour and quality are factors not so easily obtained when gathering second-hand materials, as will be shown in the case of Maubrey's work, but in Wynne's case it seems to be a necessity. 


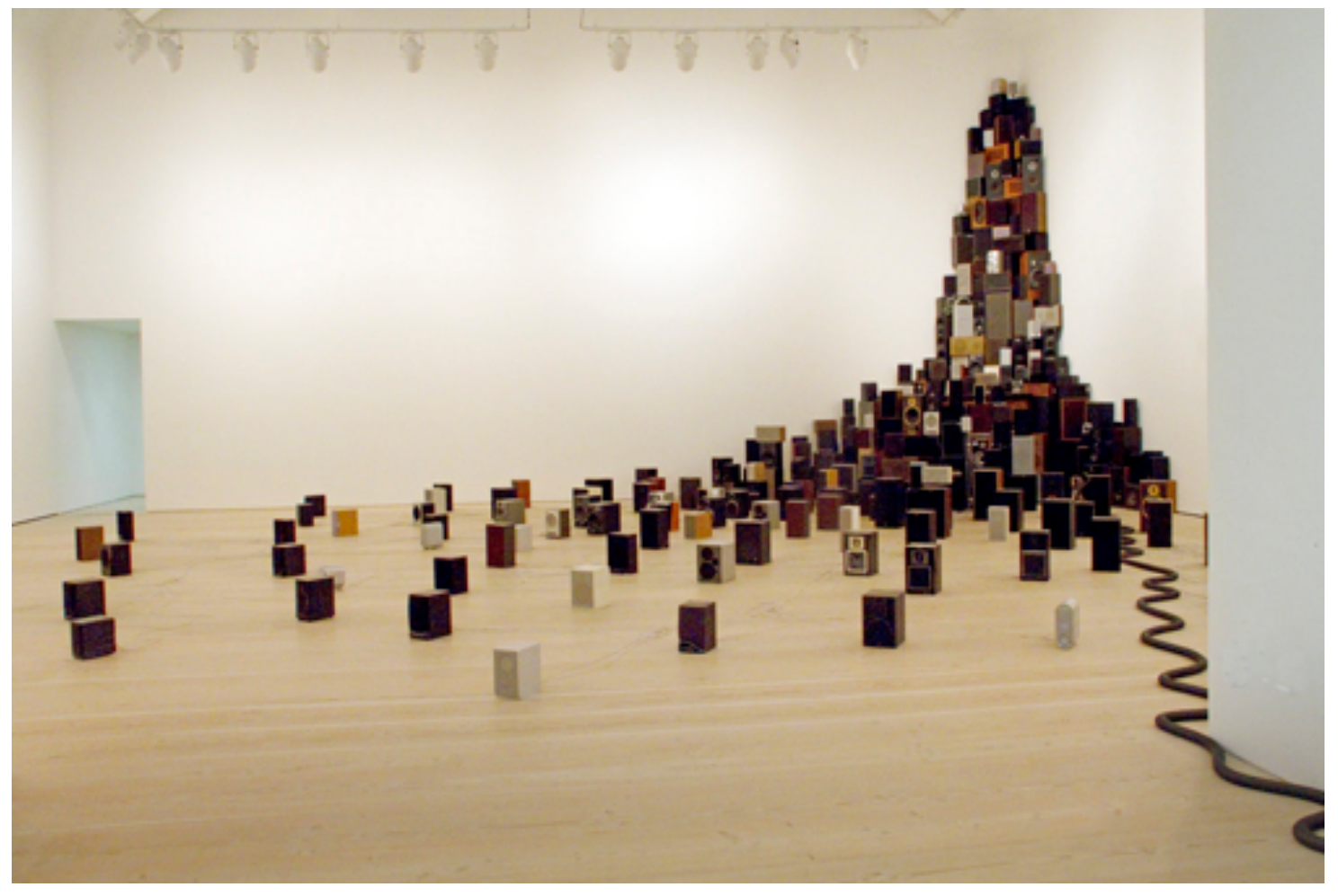

Figure 13. John Wynne's Installation for 300 speakers

As is the case in all of Wynne's works, the shape of the setup is composed to activate and enhance aural architecture ${ }^{17}$. The 'mountainous formation of speakers' in the work Installation for 300 speakers, pianola and vacuum cleaner (2009) 'functions both visually and as a platform for the projection of sound' (Wynne, 2010). The Research $\log ^{18}$ for this piece shows at least five of the possible setups for the installation, which were carried out in test before the final rendition. These were discarded due to both visual and aural impact or lack thereof. The final result sees the tower of 300 loudspeakers nearly reaching the ceiling in the corner of the Saatchi Gallery in London. In a semi-pyramidal setup the tower becomes wider from the tip, until the loudspeakers reach the floor and are spread across the gallery. In this setup the loudspeaker becomes inaccessible at the physical peak of the installation. In Wynne's other two installations described here, the majority of the loudspeakers are placed upon the floor in direct physical proximity to the audience, whereas 300 Speakers places the object beyond our

\footnotetext{
${ }^{17}$ See Blesser, 2007

${ }^{18}$ See Wynne, 2009
} 
grasp. This inaccessibility is consequential within large-scale works. Engaging with the individual loudspeaker, aurally or visually, is made more difficult as the size of the installation expands. The focus shifts and becomes more about the physical composition of the objects, not whether we are able to receive valid sensory information from them.

\subsection{Benoit Maubrey}

Highlighting the extreme end of the scale, with regard to these artists, is Maubrey's body of work. Maubrey's installations make use of vast numbers of loudspeakers. The smallest of these works, Audio Igloo (1997), has three hundred loudspeakers, whilst the largest work, Temple (2012), consists of three thousand loudspeakers. The installations here call to attention aspects of logistics, construction, time, effort as well as the patience needed to collect the materials and create arrangements so monumental.

In a contrast to Wynne's aesthetic, the loudspeakers in Maubrey's work take on a distinctly 'as is' quality. This is not just about availability of materials as one may suspect, but is about a distinct visual aesthetic. Verbeek says this about personal selection: 'If there is any originality and uniqueness in the objects that surround us, it lies in the selection we have made...rather than in the objects themselves, of which there are thousands of other identical examples' (Verbeek, 28-9). Although both artists make use of second hand materials there are definite unique characteristics each artist makes use of within their work. Maubrey's work highlights the body of the loudspeaker along with all its flaws. Loudspeakers with tears, rips and dents are all used, none being discarded for non-conformity to a specific aesthetic. In this sense Maubrey is more partial to quantity over quality, especially in comparison to Wynne. 


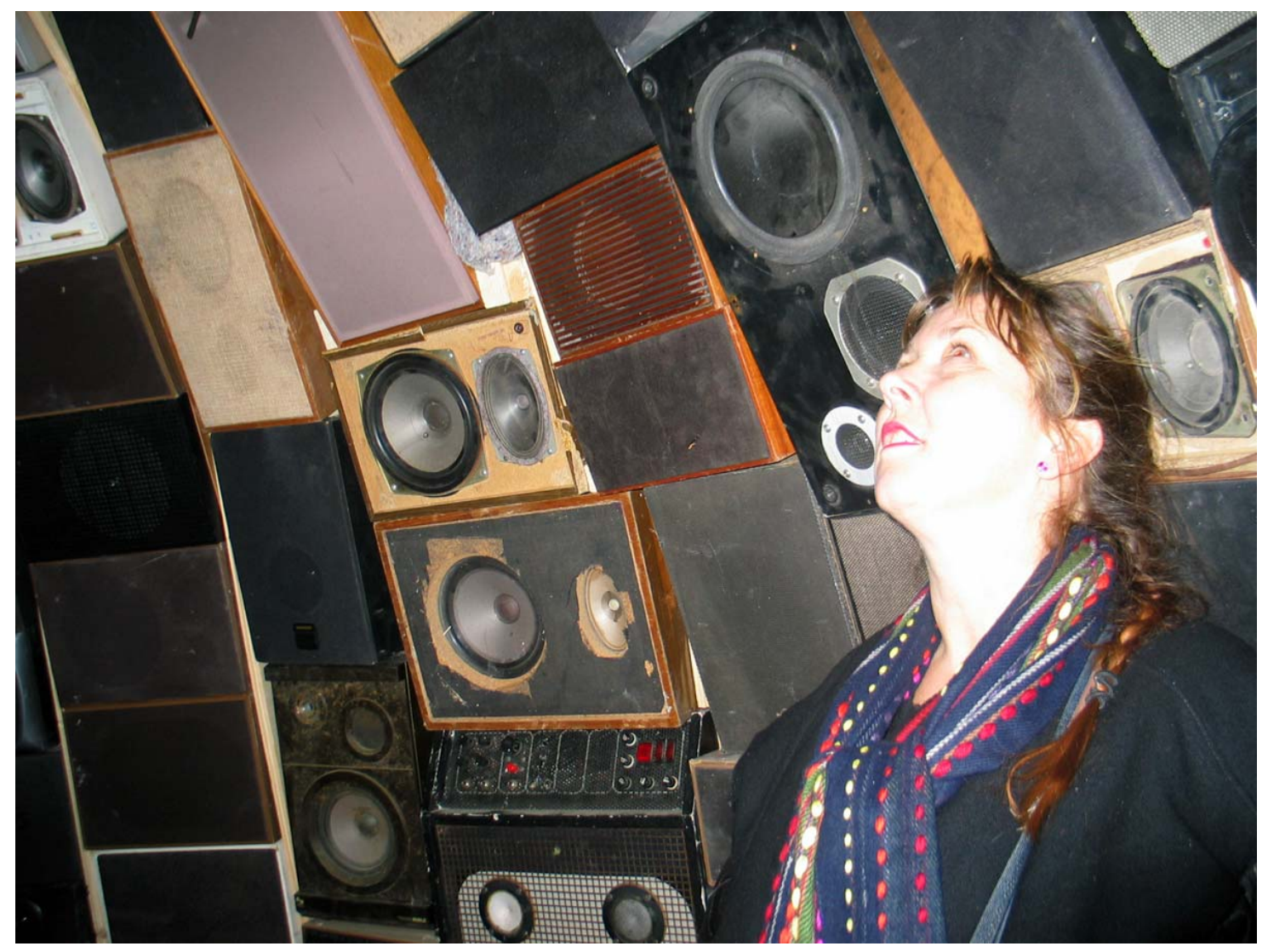

\section{Figure 14. Benoit Maubrey's Audio Igloo}

This concept of quantity over quality can be seen immediately in Audio Igloo, as close up pictures of the installation reveal the levels of deterioration of some of the loudspeakers. The loudspeakers in this piece are setup in the shape of an igloo large enough to hold several people. When inside the sculpture the listener is nearly completely encased, apart from the floor and the entranceway. Radiating white noise, the audible and visual spectrum is full. Another work of Maubrey's that utilises white noise is the work Speakers Gate (2010). This sculpture of 350 loudspeakers is a 'replica of the gateway of a 6th century fortress (the KORSE)' (Maubrey, 2011b) and is created out of solid black loudspeakers. Viewed from a distance, this installation may be perceived as a solid structure, where the sonic element may have little or no meaning. Upon a closer look a shift takes place where the structure reveals itself to be made up of these smaller objects. The sculpture then has the possibility of taking on a more functional and active role, not only perceptually but also aurally. 
Maubrey's largest works also replicate, or show similarity to, extant physical structures. Speakers Wall-Le Mur Sonore (2011) is a towering wall of loudspeakers that is juxtaposed and matched in size with an original piece of the Berlin wall. This massive sculpture consists of '1000 recycled loudspeakers, radios and amplifiers' (Maubrey, 2012a). In contrast to Wynne's work 300 Speakers, where the size of the installation is constrained by the space, the size of Maubrey's sculpture has direct conceptual significance, which is its comparison to the section of the Berlin wall. Speakers Wall is visually dense and the extreme range of loudspeakers in the setup requires perceptual unpacking: where did the loudspeakers come from? How long did it take to setup? The vast number of shapes within the structure, circles and rectangles, looming and glaring at the audience become as overwhelming as the Berlin wall itself would have been for so many. Taking the installation in light of its socio-political bonds, the loudspeakers may come to represent 'speakers' or people from both sides of the wall: those in protest and those in authority. 


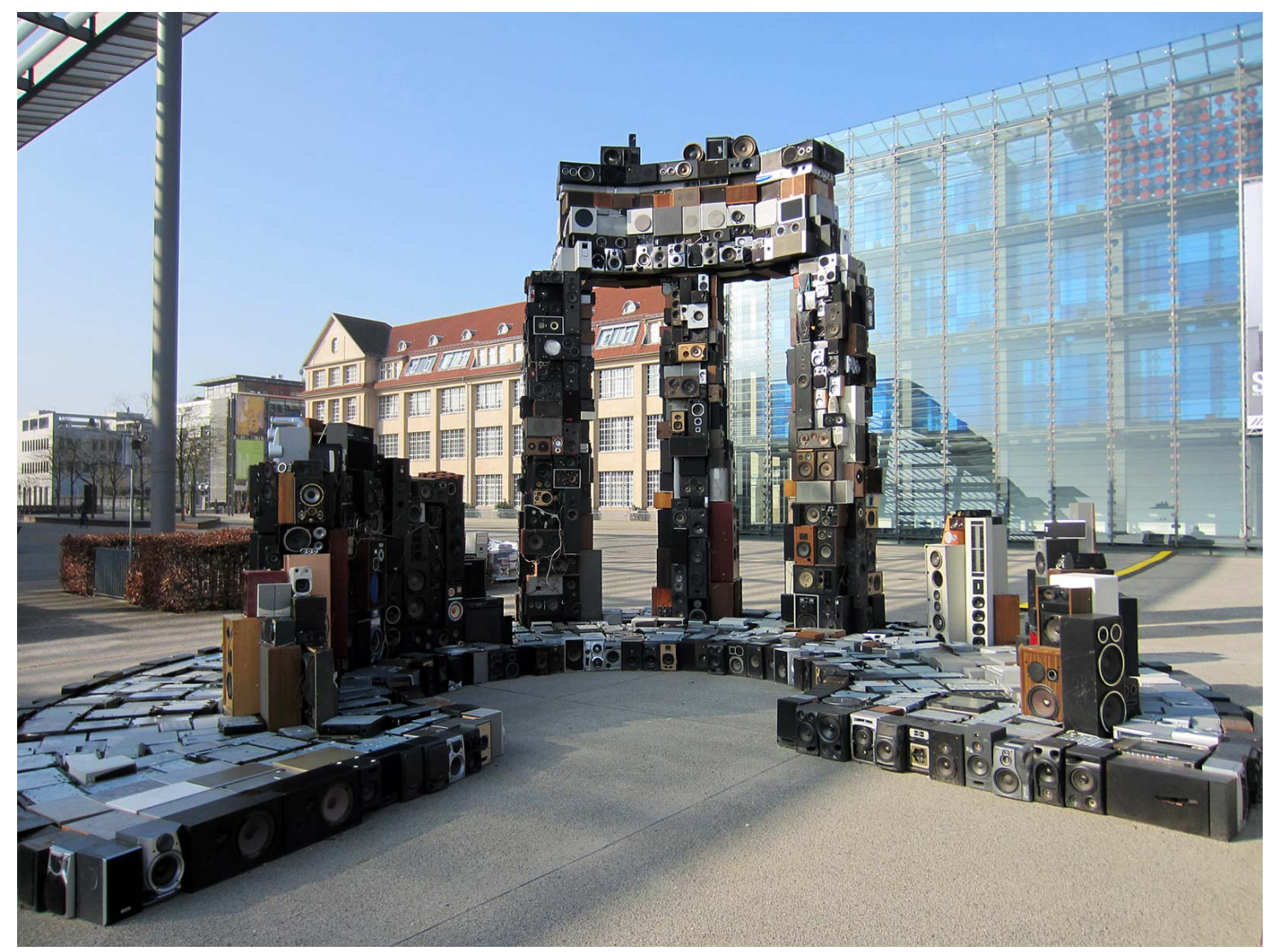

\section{Figure 15. Benoit Maubrey's Temple}

The largest of Maubrey's sculptures Temple increases the number of loudspeakers to a maximum in his work. This replica of the Greek Tholos temple in Delphi ${ }^{19}$ is made of three thousand recycled loudspeakers. This particular way of representing a physical structure calls into question the significance of the original structure and the nature of the object used to replicate it. In these works the original structure may take on specific geographical significance for the composer or the audience, bringing in the element of site specifics. This is then contrasted with the seemingly universal nature of the loudspeaker, where potential meaning and interpretation on the part of the audience is limitless. Taking this into account, audience interpretation becomes an all-important factor. The level of engagement Maubrey seeks for his works is shown in the sonic content of Speakers Wall and Temple, where he sets up a phone line to run directly

\footnotetext{
${ }^{19}$ A sacred place for communicating with the Gods, the symbol for contact beyond the realms of people's imagination. (Maubrey, 2012b)
} 
through the sculpture. By calling the specified number, audience members can participate in the work for three minutes placing their own personal imprint upon the work.

In these and other large-scale loudspeaker installations, an awkward tension is setup. The traditional relationship between the listener and the object is broken down not only physically but also aurally. Tension develops as visually we may move past the individual object toward the meta-object. This invalidates the individual object's original function in submission to that of its function within the sculpture. As the visual overwhelms the aural, perceptual proportions slip, with the aural being subsumed by the visual and physical. My work Jukebox, by contrast, provides multiple instances of the object, but does so in a way that provides access, both physically and aurally to each item: the top of the sculpture remains at head height. Similarly, in Osborn's work the sounding and visual components seem to find a natural equilibrium where one side is not validated without the other, in which the relationships between the technology and the sound are clear. In the larger works discussed, the lack of an aural component would still provide ample information to provide the audience with an engaging, although single-sided, experience. The degree to which these pieces are a spectacle is just as important, if not more, than providing a complex conceptual construct or compelling sonic material. Speakers Wall and Temple exemplify this accentuation of the structure at the potential expense of sonic content, creating a complete shift in the nature and use of the loudspeaker. 


\section{Conclusion}

In conclusion, this exegesis shows the loudspeaker to be a rich and complex object. Functionally it has many transformative properties that affect sound and music. It also has the potential to be used as a tactile instrument, not only transforming sound, but also creating sound. I have taken a reduced look at the object and mechanism at some points in this exegesis, but overall the loudspeaker provides us with a plethora of information and is active upon our perceptions both aurally and visually. As an artifact, the object creates and shapes meaning for the listener and user, both inside and outside sonic contexts, and at times it also transcends its function to become an object of fetish and commodity.

As this exegesis mainly concentrates on the artistic facets of the object, analyses of other areas of its use were excluded. For this reason there is strong scope for developing this project further. For example, one side of the object that I have not explored is the socio-political use and function of the loudspeaker. Preliminary research has shown that the ways in which the object has been used in this respect, including but not limited to its use in propaganda and torture ${ }^{20}$, suggests a scope as far ranging as the technological developments it has undergone. Exploration of this side of the topic was outside the direct scope of this exegesis but there is room for further examination. My Future work may include examination of the socio-political dimensions of the object through my practice-based research. This would lead me into territory where referential sonic material and semantic content would be utilized, contrasting with my recent work, where the audible content is utilized in a reduced ways. Beyond this, the potential for the loudspeaker to be developed as an instrument has been made clear. My aim is to

${ }^{20}$ See Goodman, 2010 
continue to expand and refine my practice for the loudspeaker-as-instrument, using it to actively create sonic content both within a solo context and within an ensemble. 


\section{Bibliography}

Adorno, Theodore W. (1941). "The Radio Symphony: An experiment in theory”, in Essays on Music, Berkeley, Calif. : University of California Press, 2002: pp. 251-269.

Ashby, Arved (2010). Absolute Music, Mechanical Reproduction, Berkeley, University of California Press.

Barthes, Roland (1977). “The Death of the Author”, in Image, Music, Text, trans Stephen Heath, New York, N.Y. : Noonday Press, 1988.

Blesser, Barry and Salter, Linda-Ruth (2007). Spaces speak, are you listening? : experiencing aural architecture, Cambridge, Mass. : MIT Press.

Brey, Philip (2000). Technology and Embodiment in Ihde and Merleau-Ponty, Metaphysics, Epistemology, and Technology. Research in Philosophy and Technology, vol 19. ed. C. Mitcham. London: Elsevier/JAI Press.

Carpenter, Patricia (1967). “The Musical Object”, in Current Musicology, issue 5 pp. 56-87, 1967,

<http://search.proquest.com.helicon.vuw.ac.nz/docview/907304659?accountid=14782> (28 May 2012)

Chion, Michel and Brewster, Ben (1991). Quiet Revolution... And Rigid Stagnation, October, Vol. 58, Rendering The Real (Autumn, 1991), pp. 69-80, MIT Press.

Chion, Michel (1994). Audio-Vision: Sound on Screen, trans. Claudia Gorbman, Columbia University Press, New York.

Coleman, Mark (2004). Playback: from the Victrola to MP3, 100 years of music, machines, and money, New York : Da Capo Press. 
Debord, Guy (1994/ 1967). The Society of the Spectacle, trans. Donald NicholsonSmith, New York: Zone Books.

Dre. Dr. (2011). <http://www.beatsbydre.com/about/about.aspx http://www.beatsbydre.com/Default.aspx> (28 May 2012).

Eisenberg, Evan (2005). The Recording Angel: music, records and culture from Aristotle to Zappa, New Haven, CT : Yale University Press.

Ellen, Roy (1988). Fetishism, Man, New Series, Vol. 23, No. 2 (Jun., 1988), pp. 213 235.

Emmerson, Simon (2000). Introduction, in Emmerson, Simon (ed), Music, Electronic Media And Culture, pp.193-216, Ashgate Publishing Limited, England.

Friedl, Reinhold (2002). Some Sadomasochistic Aspects of Musical Pleasure, Leonardo Music Journal, Vol. 12, Pleasure, pp. 29-30.

Goodman, Steve (2010). Sonic Warfare, The MIT Press Cambridge, Massachusetts London, England.

Gramophone Magazine (1994): Volume 71, Number 851; Gramophone, General Gramophone Publications Limited, Middlesex, April, 1994.

Helmholtz Resonance. <http://www.phys.unsw.edu.au/jw/Helmholtz.html> (28 May 2012).

Henriques, Julian (2011). Sonic Bodies: Reggae Sound Systems, Performance Techniques and Ways of Knowing, New York : Continuum.

Ihde, Don (2007). Listening and Voice: Phenomenologies of Sound, State University of New York Press, Albany. 
Jarre, Jean Michel (2011). <http://www .jarre.com/aerodream-one-ipod $®$-iphone $₫$ dock-speaker> (28 May 2012).

Kelly, Caleb (2009). Cracked Media: the sound of malfuntion, Cambridge, Mass. : MIT Press.

Labelle, Brandon (2006). Background Noise: Perspectives on Sound Art, New York : Continuum International.

Leiss, William (1990). Under Technology's Thumb, Montreal : McGill-Queen's University Press.

Marcuse, Herbert (1964). One Dimensional Man; studies in the ideology of advanced industrial society, Boston, Beacon Press.

Maubrey, Benoit (2011a). Audio Igloo, <http://www.benoitmaubrey.com/?p=90> (28 May 2012).

Maubrey, Benoit (2011b). Speakers Gate, <http://www.benoitmaubrey.com/?p=739> (28 May 2012).

Maubrey, Benoit (2012a). Speakers Wall, <http://www.benoitmaubrey.com/?p=820> (28 May 2012).

Maubrey, Benoit (2012b). Temple, <http://www.benoitmaubrey.com/?p=1963> (28 May 2012).

McGinn, Robert E (1983). Stokowski and the Bell Telephone Laboratories:

Collaboration in the Development of High-Fidelity Sound Reproduction, Technology and Culture, Vol. 24, No. 1 (Jan., 1983), pp. 38-75.

McKinnon, Dugal (2011). <http://dugalmckinnon.wordpress.com/2011/06/14/noncochlear-sound-art/> (28 May 2012). 
New Oxford American Dictionary (2005). 2nd edition, Oxford University Press, Inc.

Nowak, Nik (2011). <http://www.freshnessmag.com/2011/09/28/nik-nowak-panzersound-tank-4000-watts-of-pure-hi-fi-assault/> (28 May 2012).

Osborn, Ed (2012a). Flying Machines, <https://vimeo.com/3962533> (28 May 2012).

Osborn, Ed (2012b). Swing Set, <https://vimeo.com/3998028> (28 May 2012).

Perich, Tristan (2005-2010). Breathing Portraits,

<http://www.tristanperich.com/\#breathing_portraits> (28 May 2012).

Perlman, (2004). Golden Ears and Meter Readers: The Contest for Epistemic Authority in Audiophilia, Social Studies of Science, Vol. 34, No. 5, Special Issue on Sound Studies: New Technologies and Music (Oct., 2004), pp. 783-807.

Ponty, Merleau (1958/1945). Phenomenology of Perception, trans Routledge \& Kegan Paul, New York : Routledge.

Raaymakers, Dick (2012a). Ideophone 1, <http://www.v2.nl/archive/works/ideophone1> (28 May 2012).

Raaymakers, Dick (2012b). Intona: dodici manieri di far tacere un microfono (twelve ways to make a microphone shut up), <http://www.v2.nl/archive/works/intona> (28 May 2012).

Richardson, Mark (2012). Resonant Frequency Permission to Avalanche:

How bass affects our bodies, from Dr. Dre's headphones to Aphex Twin, January 20, $2012<$ http://pitchfork.com/features/resonant-frequency/8757-permission-to-avalanchefour-fragments-on-bass/> (28 May 2012). 
Shaw, Debra. Benita (2008). Technoculture: The Key Concepts, Oxford; New York: Berg.

Smalley, Dennis (2001). Spectromorphology: Explaining sound-shapes, Organised Sound/ Volume 2/ Issue 02, pp107-126 Published online: 19 July 2001.

Stanford Encyclopaedia of Technology (2006). typel token, $<$ http://plato.stanford.edu/entries/types-tokens/> (28 May 2012).

Transmission Audio (2010a). <http://www.transmissionaudio.com/home.html > (28 May 2012).

Transmission Audio (2010b). Ultimate, <http://www.transmissionaudio.com/images/Transmission_Audio_ULTIMATE.pdf > (28 May 2012).

Verbeek, Peter-Paul (2005). What Things Do: Philosophical reflections on technology, agency, and design, University Park, Pa. : Pennsylvania State University Press.

'Wall of Sound' (2012). <http://www.dozin.com/wallofsound/> (28 May 2012).

Whitelaw, Mitchell (2001). Stacker, Catalogue Essay, <http://creative.canberra.edu.au/mitchell/papers/Stacker.pdf> (28 May 2012).

Wynne, John (2006). 230 Unwanted Speakers, <http://www.bbc.co.uk/humber/content/articles/2006/05/12/230_unwanted_speakers.sht $\mathrm{ml}>$ (28 May 2012).

Wynne, John (2010). <http://www.sensitivebrigade.com/untitled.htm> (28 May 2012).

Wynne John (2004). Acoustic Ecology, <http://www.sensitivebrigade.com/Fallender_Soundscape.html> (28 May 2012). 
Wynne, John (2009). Research log,

<http://www.sensitivebrigade.com/Research_log.htm> (28 May 2012).

\subsection{Works Cited}

Flanigan, Lesly (2009). Lesley Flanigan at Bent festival 2009,

<https://vimeo.com/16851981> (28 May, 2012).

Lynch, David (2002). Eraserhead, (1977), Digitally Remastered Special Edition, Umbrella Entertainment.

Maubrey, Benoit (2011a). Audio Igloo (1997),

<http://www.benoitmaubrey.com/?p=90> (28 May 2012).

Maubrey, Benoit (2011b). Speakers Gate (2010),

$<$ http://www.benoitmaubrey.com/?p=739> (28 May 2012).

Maubrey, Benoit (2012a). Speakers Wall (2011),

<http://www.benoitmaubrey.com/?p=820> (28 May 2012).

Maubrey, Benoit (2012b). Temple (2012), <http://www.benoitmaubrey.com/?p=1963> (28 May 2012).

Merzbow (2005). Merzbuddha, Important Records - imprec052, 2005.

Monahan, Gordon (1982). Speaker Swinging (1982),

<http://www.gordonmonahan.com/pages/speaker_swinging.html> (28 May 2012)

Osborn, Ed (2012a). Flying Machines (2002), <https://vimeo.com/3962533> (28 May 2012).

Osborn, Ed (2012b). Swing Set (2002), <https://vimeo.com/3998028> (28 May 2012). 
Perich, Tristan (2010). Breathing Portraits (2005-2010), <

https://vimeo.com/15259734> (28 May 2012).

Raaymakers, Dick (2012a). Ideophone 1 (1970),

<http://www.v2.nl/archive/works/ideophone-1> (28 May 2012).

Raaymakers, Dick. Intona: dodici manieri di far tacere un microfono (twelve ways to make a microphone shut up) (1992), <http://www.v2.nl/archive/works/intona> (28 May 2012).

Schaeffer, Pierre (1948). "Etude Aux Chemins De Fer", in Various - Early Modulations Vintage Volts, Caipirinha Productions - CAI-2027-2, 2000.

Wynne, John (2006). 230 Unwanted Speakers (Walnut Grained Vinyl Veneered Particleboard Construction) (2006), <http://www.sensitivebrigade.com/Hull.htm> (28 May 2012).

Wynne, John (2004). Fallender ton für 207 lautsprecher boxen (Falling tone for 207 speakers) (2004), <http://www.sensitivebrigade.com/Fallender_ton.htm> (28 May 2012).

Wynne, John (2010). Installation for 300 speakers, pianola and vacuum cleaner (2010), <http://www.sensitivebrigade.com/untitled.htm> (28 May 2012)

\subsection{Figures}

Figure 1. Nik Nowak's Soundtank, <http://www.freshnessmag.com/2011/09/28/niknowak-panzer-sound-tank-4000-watts-of-pure-hi-fi-assault/> (28 May 2012).

Figure 2. Jamaican Dancehall Sound-System, <http://www .troubadourblues.com/2010/11/reflections-on-global-reggae/> (28 May 2012).

Figure 3. Lesly Flanigan's Live Performance, <http://disquiet.com/2010/01/08/lesleyflanigan/> (28 May 2012). 
Figure 4. Gordon Monahan's Speaker Swinging (1982),

<http://www.gordonmonahan.com/pages/speaker_swinging.html> (28 May 2012).

Figure 5. Ed Osborn's Flying Machines (2001), <https://vimeo.com/3962533> (28 May 2012).

Figure 6. Blue (2012), Jason Wright (2012).

Figure 7. Dick Raaymakers' Intona (1992), <http://www.v2.nl/files/1992/intonadocumentation/56.jpg/view> (28 May 2012).

Figure 8. Transmission Audio's 'In-wall design. Can you see the speakers...?', $<$ http://www.transmissionaudio.com/> (28 May 2012).

Figure 9. Sean Kerr's Stacker (2001), <http://www.seankerr.auckland.ac.nz/> (28 May 2012).

Figure 10. Jukebox, Jason Wright (2011).

Figure 11. Jean Michel Jarres'Aero Dream One, <http://www.jarre.com/aerodreamone-ipod $\AA$-iphone $®$-dock-speaker> (28 May 2012).

Figure 12. The Grateful Dead's 'Wall of Sound', $<$ http://media.photobucket.com/image/wall+of+sound+grateful+dead+/Ibex2912/Vintag e/Wall\%20of\%20Sound/2296990852_61cb0e4f84_o.jpg> (28 May 2012).

Figure 13. John Wynne's Installation for 300 speakers

<http://www.sensitivebrigade.com/untitled.htm> (28 May 2012).

Figure 14. Benoit Maubrey's Audio Igloo, http://www.benoitmaubrey.com/?p=90 (28 May 2012) . 
Figure 15. Benoit Maubrey’s Temple, http://www.benoitmaubrey.com/?p=1963 (28 May 2012. 


\section{Section B: Portfolio}

\section{Overview of Practical Work}

This portfolio consists of documentation of sound installations and performance pieces completed during the course of my MMA. These pieces explore and exploit the psychoacoustic effects of the loudspeaker taking into account how the listener both views and listens to the object. Whilst some pieces have a strong conceptual dimension, others are reductive in the sense that they are exploring the physical mechanism and nature of the object.

Each work is explained in terms of the creative intentions and methods that informed its production. In most instances the works themselves are also discussed, with a wider frame of reference, in the exegesis. The reader is referred to this discussion in the following explanatory documents.

\subsection{Overview of Sound Material}

In examples of my own work I have had to create or choose sound that draws attention to and allows emphasis on the "sound of the sound" ${ }^{1}$ Intrinsic qualities of the sound become all-important as focus on extrinsic qualities may detract from the purpose of the work. One way to achieve this is to create a consistent or repeating sound input. Short sound files are created and looped to allow the listener to discern, consciously or not, the 'original' sound of the loop. This is then reproduced through the technology and the resulting transformations of the sound are gained through the physical tempering of the mechanics and body of the said objects. Through this technique identical input creates differing results. An important choice for sound materials was to make sure external references are kept to a minimum.

\footnotetext{
${ }^{1}$ Discussed in Chapter three of exegesis, Sound of the Sound
} 
In some instances careful composition of sound materials helps to achieve this. In other instances the multiple elements of the work are balanced; aural, visual and conceptual components come together to aid perceptual of the audible output.

Within my portfolio of work I have examples of using my own composition and prerecorded work. Within these examples there are points where the music is intended to sit in the foreground of the work and others where they take a background role allowing for the conceptual element and tactile application of the loudspeaker to be assessed more fully. 


\section{DVD Contents}

\subsection{Works}

Allele.mp4

Blue.mp4

Deaf of the Author.mp4

Glass.mp4

Jukebox.mov

Tender Suffocation.mp4

\subsection{Supplementary Material}

This folder contains supplementary viewing material for Deaf of the Author. Also included here are PDF files containing further pictures from my work.

Allele.pdf

Blue.pdf

Deaf Information Printout.pdf

Deaf of the Author.pdf

Deaf Video Component.mov

Glass.pdf

Jukebox.pdf

Tender Suffocation.pdf 
2.3 Electronic Copy of Exegesis

WrightJason-exegesis.pdf 


\section{Table of Figures}

Figure 1. Allele Detail 1 .$P g .89$

Figure 2. Allele Technical Setup ....................................................................9. 92

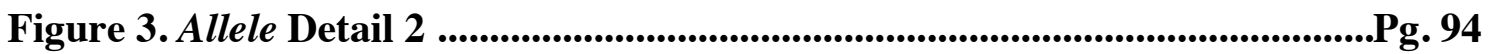

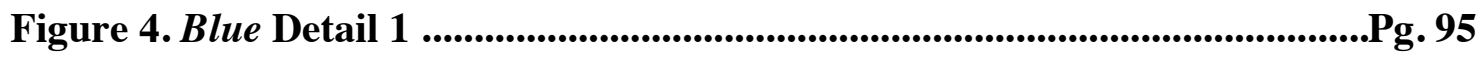

Figure 5. Blue Detail 2 ..................................................................................................Pg. 100

Figure 6. Glass Detail 1 ..............................................................................................Pg. 101

Figure 7. Glass Technical Setup .............................................................................Pg. 104

Figure 8. Glass Detail 2 .....................................................................................Pg. 105

Figure 9. Tender Suffocation Detail 1 .............................................................................Pg. 106

Figure 10. Tender Suffocation Detail 2 ..................................................................Pg. 110

Figure 11. Deaf of the Author Detail 1 .....................................................................Pg. 112

Figure 12. Deaf of the Author Detail 2 .............................................................Pg. 117

Figure 13. Jukebox Detail 1 ..................................................................................Pg. 118

Figure 14. Jukebox Detail 2 .............................................................................................Pg. 124 
4. Live Performance 


\subsection{Allele}

Live Performance, 2011

DVD - Allele.mp4

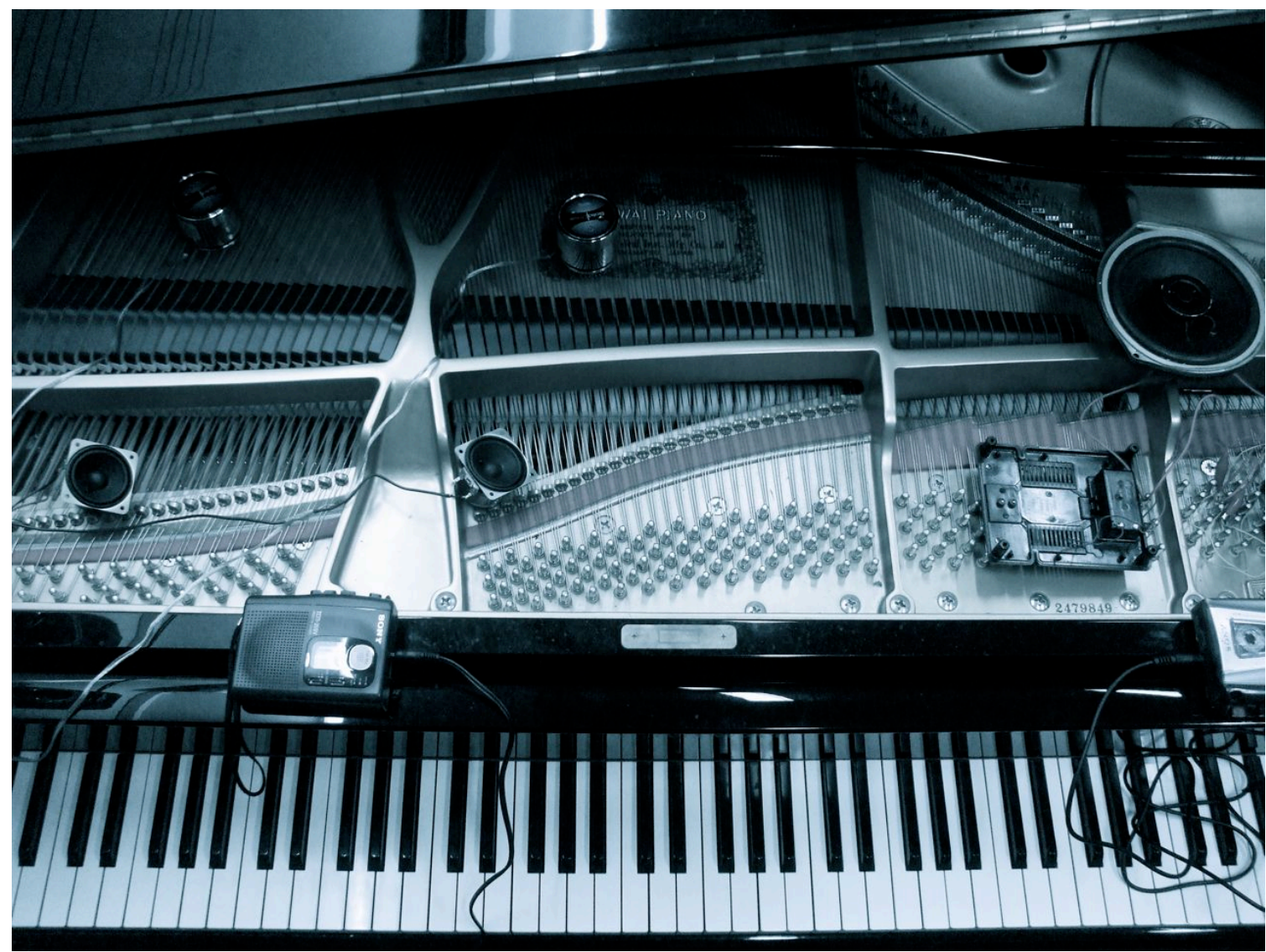

Figure 1. Allele Detail 1

\section{allele lə ' lēll}

noun Genetics

one of two or more alternative forms of a gene that arise by mutation and are found at the same place on a chromosome. ${ }^{2}$

Live Performance for Piano, Fixed media, Tape players, Loudspeakers and Solid Drive Transducers

\footnotetext{
${ }^{2}$ New Oxford American Dictionary 2nd edition (C) 2005 by Oxford University Press, Inc.
} 


\subsubsection{Equipment}

2x Large Loudspeaker Cones

2x Small Loudspeaker Cones

2x Encased Loudspeakers

2x Solid Drive Transducers

2x Cassette Players

2x Blank Cassettes

2x Stereo Amplifiers

1x Small Marshall Amplifier

$1 \mathrm{x}$ iPod

1x Grand Piano

\subsubsection{Overview}

This piece explores extended techniques of both the loudspeaker and the piano. Through a combination of pre-composed fixed-media, live piano, and live electronics Allele uses the loudspeaker to activate the piano and provide sonic material that is both intrinsic and extrinsic to its reproduction capabilities. By placing the loudspeaker within and upon the internal parts of the piano, the physical body and mechanism of the loudspeaker acts upon these areas, activating resonance and creating sound exterior to the loudspeaker and pianos capabilities.

Allele starts by depressing the sustain pedal on the piano. This is to leave the strings uninhibited to possible sympathetic resonance with sound material that is fed through its body and to leave it open to further vibration from loudspeakers which are placed upon 
the strings. After this, pre-composed material, derived from processed piano samples, is fed into the two encased loudspeakers at the rear of the piano via the first amplifier ${ }^{3}$ (see Figure 2.). By altering the amplitude of this material, the performer is able to activate vibration of the piano strings. When the second amplifier is used, producing the same audio material, this activates strings that the small cones and transducers are placed upon. This vibration of the strings becomes an integral sonic element of the piece; that is, not only is it a consequence of the physical bodies of the loudspeakers, but is also a feature that the composer seeks to accentuate.

As the live piano enters into the piece, particular notes are struck that have a cone or transducer on the corresponding string. This significantly alters the timbre of the note, creating a buzzing, inharmonic sound. On particular notes, the weight of the loudspeaker fully dampens the sting creating a muted and muffled version of the note. Due to the physical placement of the object upon the string, separate to its sonic output, the loudspeaker creates a clearly audible change in sound, which again is an integral part of the sonic world of the piece.

While this live section is played, the second tape recorder has been recording the output of the system. What has been recorded is then fed back through stereo amplifier 2 into the small cones and transducers, to produce a feedback loop of material, building upon resonance and vibration of the strings. The pitch of this material is altered via the speed control of the cassette player creating contrasting sonic information that processed, but remains referential to the original input. The sound of the pitch shift is put into focus, as is the sound of the cassette player clicking as it stops, starts and rewinds.

\footnotetext{
${ }^{3}$ Two stereo amplifiers are used to gain separate volume control over individual sets of speakers, which are placed at different points within the piano.
} 
The second tape player is put into record mode while still outputting into the $2^{\text {nd }}$ amplifier creating feedback, which fluctuates according to the level of input.

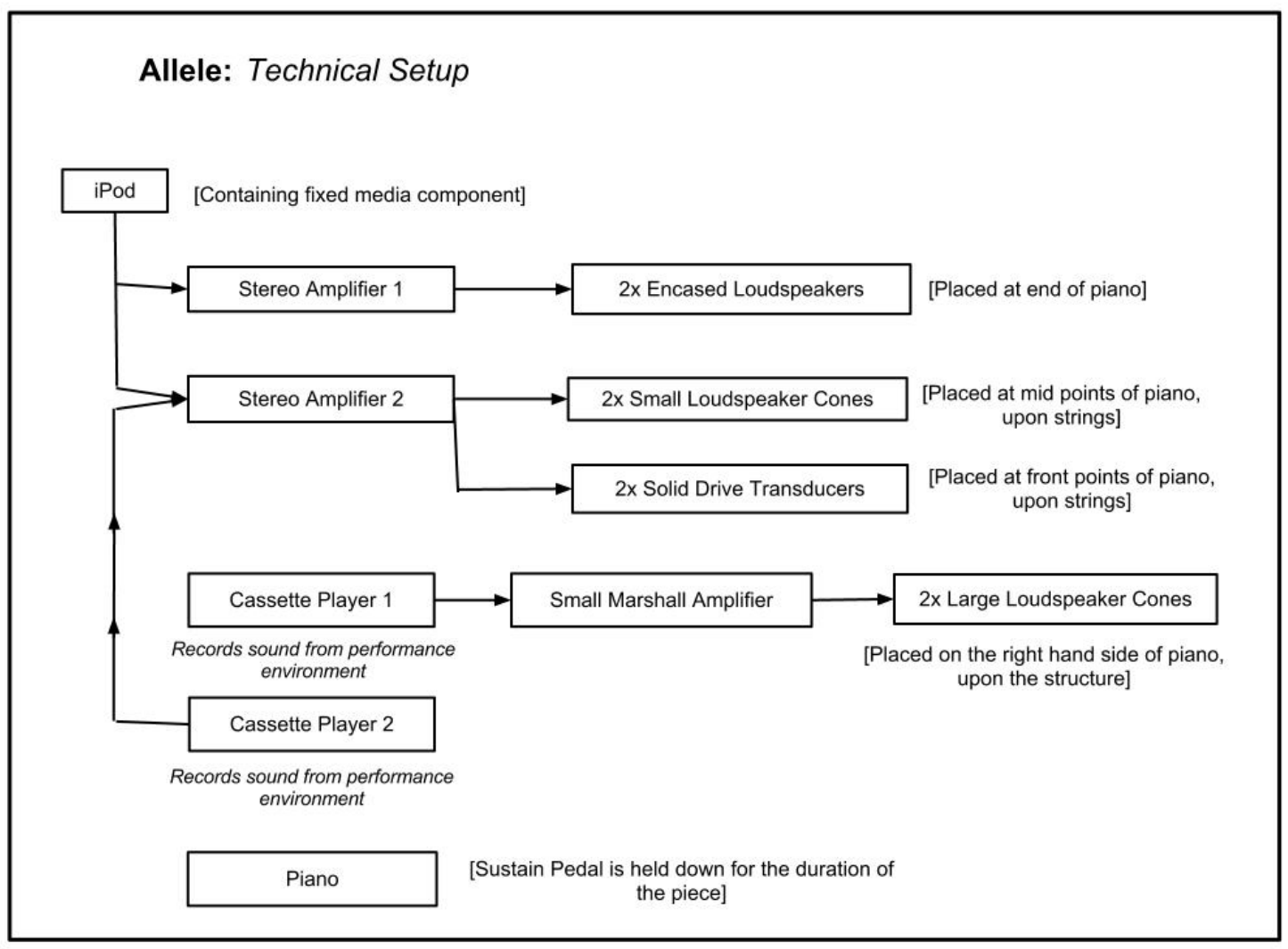

Figure 2. Allele Technical Setup

\subsubsection{Techniques}

Allele is setup to establish both a performance system and an environment, where the technology fuses to the acoustic instrument, using both aspects simultaneously to create sound. In this piece the placement of the loudspeakers within the structure of the piano does not inhibit their reproductive ability but rather allows the objects to be perceptually fused with the piano. The loudspeaker and piano can be perceived as the same entity with complementary sonic aims. My aim in this piece is to reduce the level of responsibility of the individual parts of the system, allowing each part to contribute equally to the overall sonic result. This means is that what is played on the piano or through the cassette player is not the focus of the piece, but what is the focus is how all 
the elements of technology interact with each other. Here the piano is prepared in such a way that the structure and body of the piano are utilised as elements integral to gaining the desired sonic result. By either activating resonance or vibrating against certain parts of the piano the four pairs of loudspeakers are producing their own sound and creating another layer of sonic material attributed to physical contact with the piano.

\subsubsection{Musical Material}

Allele is an improvised performance and has no score. Through experimentation and practice for the piece I began to create a compositional trajectory and dynamic, which led me to establish definite sections to move through sequentially, such as the introducing the fixed media component, playing the piano, recording with the cassette players and using the material recorded through these for the outro. Within this loose structure, timing and other factors such as pitch and rhythm played on the piano are completely improvised.

The fixed media component was created by digitally processing sampled piano material. An eight note gesture was recorded in MIDI then bounced into audio. This audio sample went through several processes such as reverb, pitch shifting, transient shifting, EQ, and filtering. These processes resulted in separate audio tracks that were layered to create the piece. The audio is eight minutes long and over the course of the main work it is looped, to be drawn upon as a sonic element when needed. My choice for using piano based material was to have sound that was to be already sympathetic and similar to the sound made within the body of the piano. Pitch shifting the material down, and amplifying higher resonant points in the recorded material allowed for a greater range of activation of sympathetic resonance. 


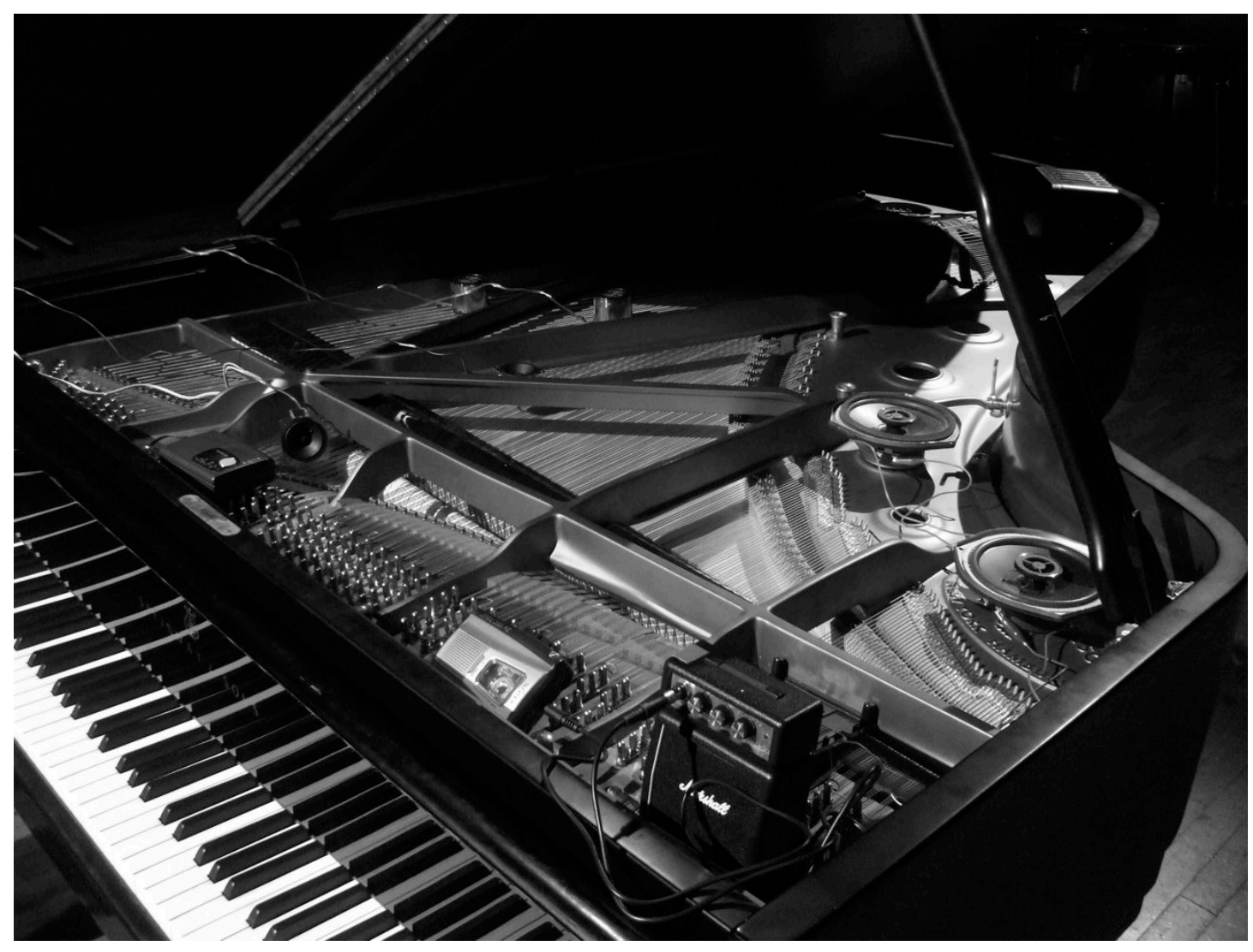

Figure 3. Allele Detail 2 


\subsection{Blue}

Live Performance, 2012

DVD - Blue.mp4

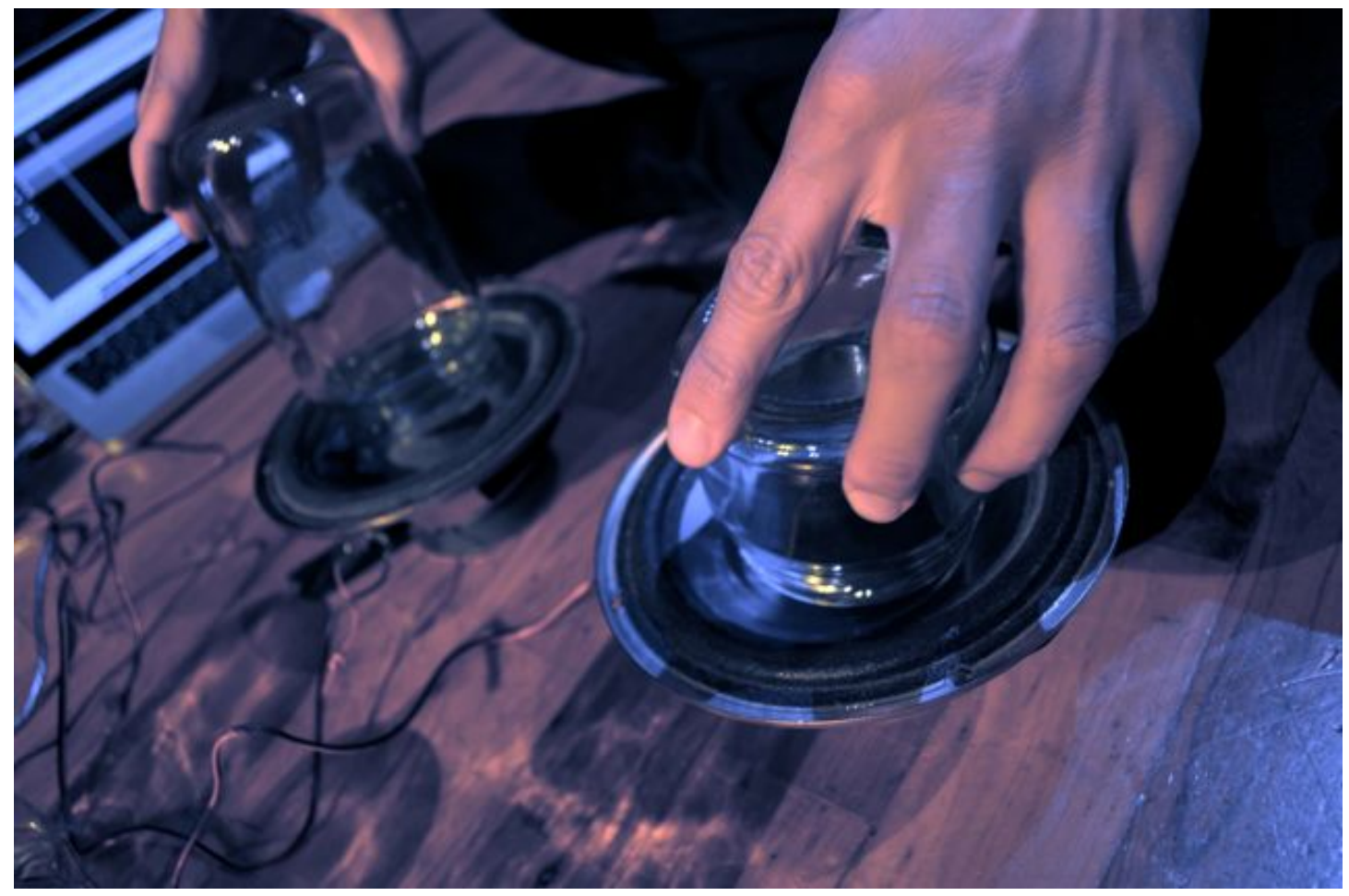

Figure 4. Blue Detail 1

Performers: Bridget Johnson, Jason Wright 


\subsubsection{Equipment}

Laptop

Stereo amplifier

Midi keyboard

2x Loudspeaker cones

1 Full frequency capable loudspeaker

5 Glass jars

\subsubsection{Overview}

Blue uses the loudspeaker as an instrument within a small ensemble, in this case a duo, where two performers improvise within the physical performance environment created by the composer. The loudspeaker/ performer amalgam here is set up to contrast with the keyboard player. By physically placing the two instruments together and showing the correlation between hand movements and resulting morphologies of sound I am suggesting that the loudspeaker can be incorporated into a more traditional view of a musical instrument. By contrasting the keyboard and the loudspeakers I am aiming to show that the two instruments have an equal sense of authorship on the resulting sound of the overall system.

In Blue a simple signal chain is setup:

Performer1/ Keyboard $\rightarrow$ Laptop (Sound Synthesis) $\rightarrow$ Amplifier $\rightarrow$ Performer2/

\section{Loudspeaker}


A third loudspeaker is introduced into the setup playing the same material that is fed through to the two performative loudspeakers ${ }^{4}$. This is used as a monitor to play the unmodified keyboard part also to fully reproduce the bass frequencies adequately. This creates a richer sound within the system and contrasts with the transformed and impure sounds gained from the performative loudspeakers.

In this piece the two performers are on opposite ends of the same signal chain, meaning the sound the second performer has access to, is determined by the first performer. Performatively speaking, once a dialogue is set up between the two performers, the second performer's actions may determine the first performer's actions, setting up a situation of mutual performative feedback and musical interaction.

\subsubsection{Performance Technique}

'The performance technique in this piece sees an object used to directly touch the loudspeaker, in this case, a glass jar. In contrast to my work Tender Suffocation (2012) where materials are placed upon the loudspeaker and left static, this piece sees me using objects in a far more active and dynamic sense to physically interact with the loudspeaker, sculpting the sounds emanating from the cones. The glass jars are used to press against the surface of the loudspeaker and are manoeuvred and positioned to create a desired sound. The moving loudspeaker cone comes into contact with the glass jar, causing it in turn to vibrate and resonate. Through movement of the jar I am able to activate resonances and harmonics that are separate to the material the loudspeaker is producing. By holding the jars upside down, I am also able to create sympathetic Helmholtz $\mathrm{z}^{5}$ resonances in the jar, by holding them slightly above the surface of the loudspeaker (this depends on the size of the jar and the note played, which I will address

\footnotetext{
${ }^{4}$ Discussed in full in Chapter Five of Exegesis, The Loudspeaker as Instrument

${ }^{5}$ A Helmholtz resonator or Helmholtz oscillator is a container of gas (usually air) with an open hole (or neck or port). A volume of air in and near the open hole vibrates because of the 'springiness' of the air inside. A common example is an empty bottle. <http://www.phys.unsw.edu.au/jw/Helmholtz.html> (28 May 2012)
} 
in the next section). There are similarities here with Lesley Flanigan's speaker performances ${ }^{6}$, where the hand is used in direct contact with the loudspeaker cone. Where this work differs is that the sound of the jars vibrating is integral to the work; in Flanigan's work, touching the loudspeaker produces different tones and levels of feedback, but the sound of the contact itself is not audible."

\subsubsection{Musical Materials}

The sound material in Blue has three components: sine tones activated by the keyboard, the pre-composed backing track and the glass jars/ loudspeaker. Sine tones were chosen for this piece for three reasons; firstly they have the function of being non-referential sound material, secondly they are able to activate the loudspeaker cone more efficiently, causing greater movement when lower frequency tones are triggered. Thirdly, pitched material is an important part of the piece, as it allows for a wider variety of timbral transformations, including activation of harmonics and resonance when touching the loudspeaker. Over the span of the keyboard, the lower half of the keyboard activates notes that are not so important in terms of providing recognisable pitch material, but rather, are used to activate the loudspeaker to increase the amount of contact with the glass jar. The upper half of the keyboard has notes that when played, have a more distinctive and recognisable pitch trajectory. These notes do not activate the cone as much as the lower tones, as the shorter frequency wavelength converts into proportionally smaller movements of the cone. Apart from choice of notes, the keyboard player has access to a 'mod wheel' on the midi controller which, when turned, applies vibrato to the sounding notes. This creates flux within any static texture that takes place as the movement of the cone flutters due to the changes in oscillation of the note.

\footnotetext{
${ }^{6}$ Explained in full in Chapter Five of Exegesis, The Loudspeaker as Instrument

${ }^{7}$ Taken from Chapter Five in Exegesis, The Loudspeaker as Instrument
} 
The pre-composed backing track is set up to add layers to both the textural and frequency components of the work and is also used aesthetically to expand the spectrum of the overall piece, contrasting with the lower sine tone material. The two elements of white noise and high iterative synthesised material oscillate between low and high amplitude periodically to create another layer of sound. Structurally, these sounds are set at a higher frequency, which activates the loudspeaker but not enough to cause vibrations with the glass jars, as does the lower frequency material. This creates a separation of the two types of materials: the functional material used to activate the loudspeaker and the purely sonic/ aesthetic layer of material.

The glass jars acting upon the loudspeakers creates the last layer of sound in the piece. These are set up in stereo, with the left loudspeaker producing the tone played on the keyboard, and the right producing a tone a third up from the original note. This allows for variation in pitch and harmonics across the two cones, even if a single note is played on the keyboard. As the loudspeaker vibrates, the jars are pressed against them, with differing amounts of pressure causing timbral change. Change in sound is also created depending on the amount of the jar's lip used; as more surface area comes into contact, the amplitude of the vibration changes.

\subsubsection{Physical Materials}

The loudspeaker cones chosen for this piece consist of a hard plastic material. This makes the contact of the jars more effective, compared to a loudspeaker with a paper cone as the more rigid material creates a higher amplitude vibration.

An aspect of the piece that brings focus to the physicality of the loudspeaker is the movement of the cone across the floor. Due to the wooden floor of the concert hall, the bottom of the loudspeaker vibrates and moves causing the performer to have to move the loudspeakers back in place. 


\subsubsection{Improvisation}

This piece has no particular compositional structure, apart from a time limit, where at 9 minutes the backing track starts to fade out. This is to signal to the performers the ending of the piece.

The instructions for the keyboard player for this piece were to improvise whilst observing the mechanics of the setup. In terms of pitch material, no particular scale was to be used, rather the pitch material was to be chromatic. Of more importance than pitch was attention to the range of notes and how these affected the loudspeakers in terms of vibration and sympathetic Helmholtz resonance in accordance with a particular jar. Combinations of single notes and chords were used, with focus not only on the audible result, but also how this acted upon the loudspeakers.

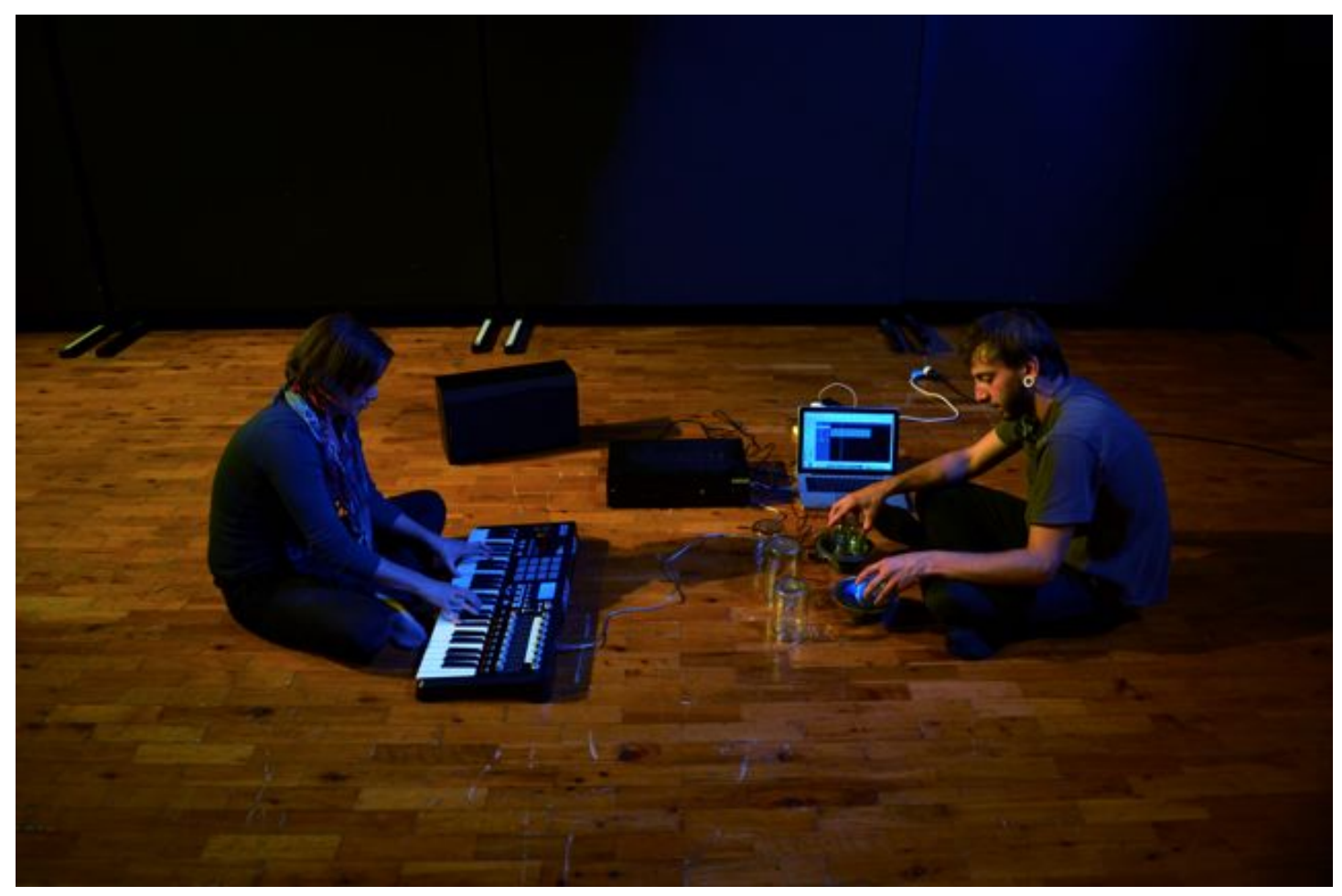

Figure 5. Blue Detail 2 


\subsection{Glass}

Live Performance, 2011

DVD - Glass.mp4

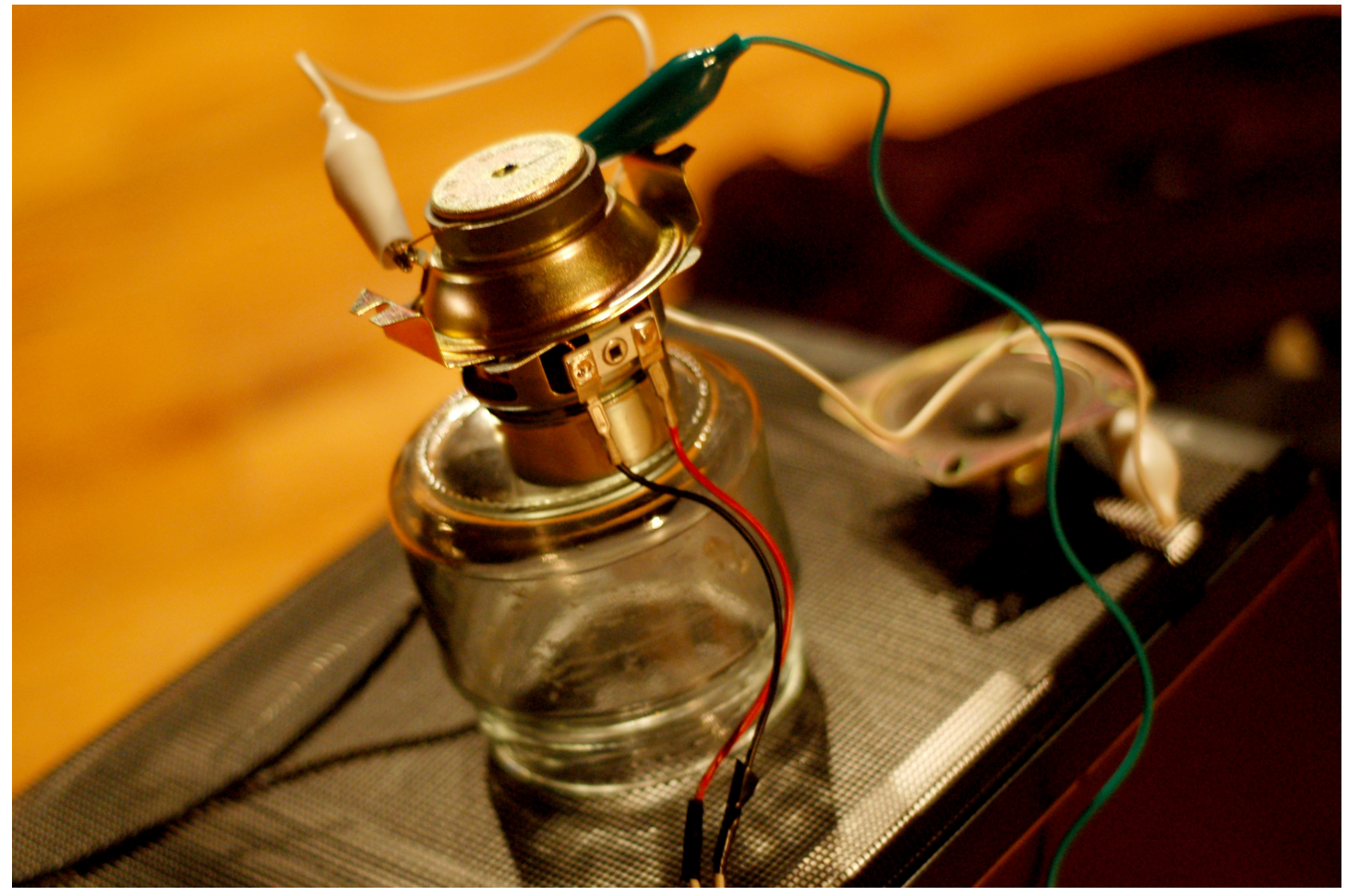

Figure 6. Glass Detail 1

Performers: seablack, Jason Wright 
This piece is an experiment in tactile applications for the loudspeaker, where the loudspeaker not only produces the sound but also is integral in generating and affecting the audio passing through the system.

\subsubsection{Overview}

Glass is an instrument for processing audio. Contained in this instrument is an amalgam of loudspeakers, various loudspeaker cones, contact microphones and glass jars. This instrument incorporates the loudspeaker as an object that does not remain passive, but becomes an active and tactile element of the work, that the performer must interact with to change the sonic result.

For this rendition of Glass there are two performers, each with their own separate system within the instrument. Both these systems are fed through the same equipment to gain similar audio results, but are exclusive, where the intrinsic factors of the audio from one system do not affect audio from the other system. Extrinsic factors such as performative communication come into play as this is a collaborative performance, but this will be addressed later.

\subsubsection{Setup}

For each system within Glass, a contact microphone is attached to a base glass jar, which is sat upon the base encased loudspeaker. This microphone is fed, through an audio interface, into logic pro, where the signal undergoes a series of digital processes. Some of these processes include, pitch shifting and harmonizing the signal. These effects are similar for each channel, but have been composed so that each channel is slightly different and has a its own sonic character. This is then routed through a stereo amplifier to either the left or right channel, where the signal if fed through the remainder of the loudspeaker cones of the system. To start creating sound the 
performers have access to a fader, which controls the gains of the contact microphone. When this fader is brought up it starts to create instant feedback because of the placement upon the base loudspeaker. The performer then has control over this feedback to prevent an overload. When this feedback first starts it has a specific tone due to the weight of the glass jar and the amount of vibration the base loudspeaker creates. This factor of feedback is utilised in Glass, using the elements of weight and vibration to further process the audio.

As the piece continues, various combinations of the loudspeakers and jars are used to vary the weight upon the jar with the contact microphone attached. This weight and placement of the loudspeakers and glass jars adds a layer of physical processing to be gained from the system. The tone and resonance of the feedback signal is changed through direct physical engagement with the objects present.

The sound of the system is self-contained, with the same loudspeakers being used to modify the signal being the ones which produce the resulting audio. By keeping the system self-contained and not linked to any external amplification a greater sense of the malleability of the sound and objects is gained. By having the dynamic of the piece subtle and not drawn completely towards the acousmatic, greater attention is brought towards the performers physical actions and the audible result of those actions. 


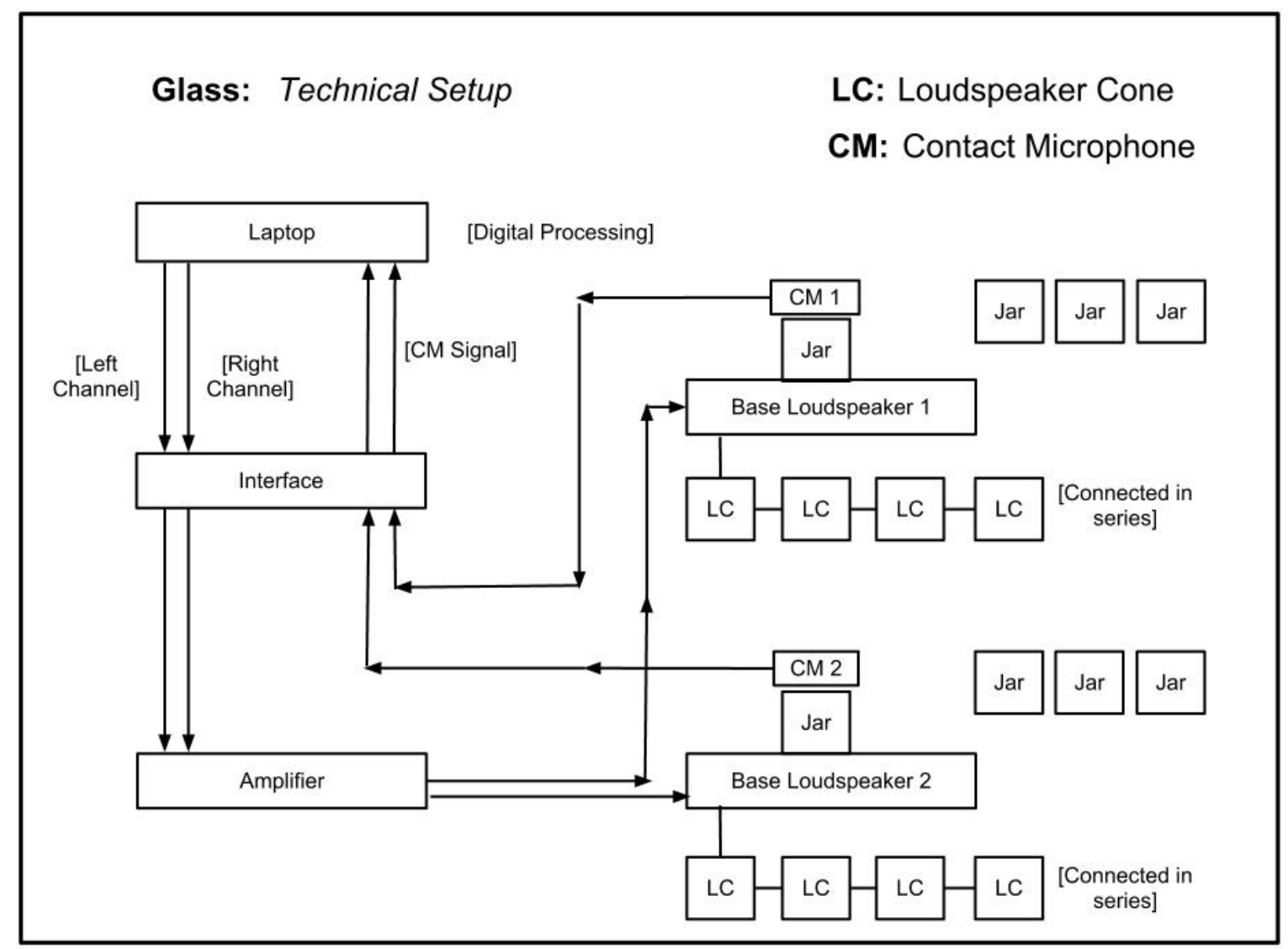

Figure 7. Glass Technical Setup

\subsubsection{Performance}

This piece has no score and is completely improvised, within the limitations of the system, whichis set by the composer. Through having two performers present, performative dialogue is setup to mirror a traditional model of improvised live music.

By having two performers improvise with a near identical set of parameters, there are benefits such as seeing a range of possible sounds, through different performance techniques of the two performers.

A more interesting performative dialogue is created that likens the performance to a more traditional model, where the nature of the system as 'instrument' is made apparent. 


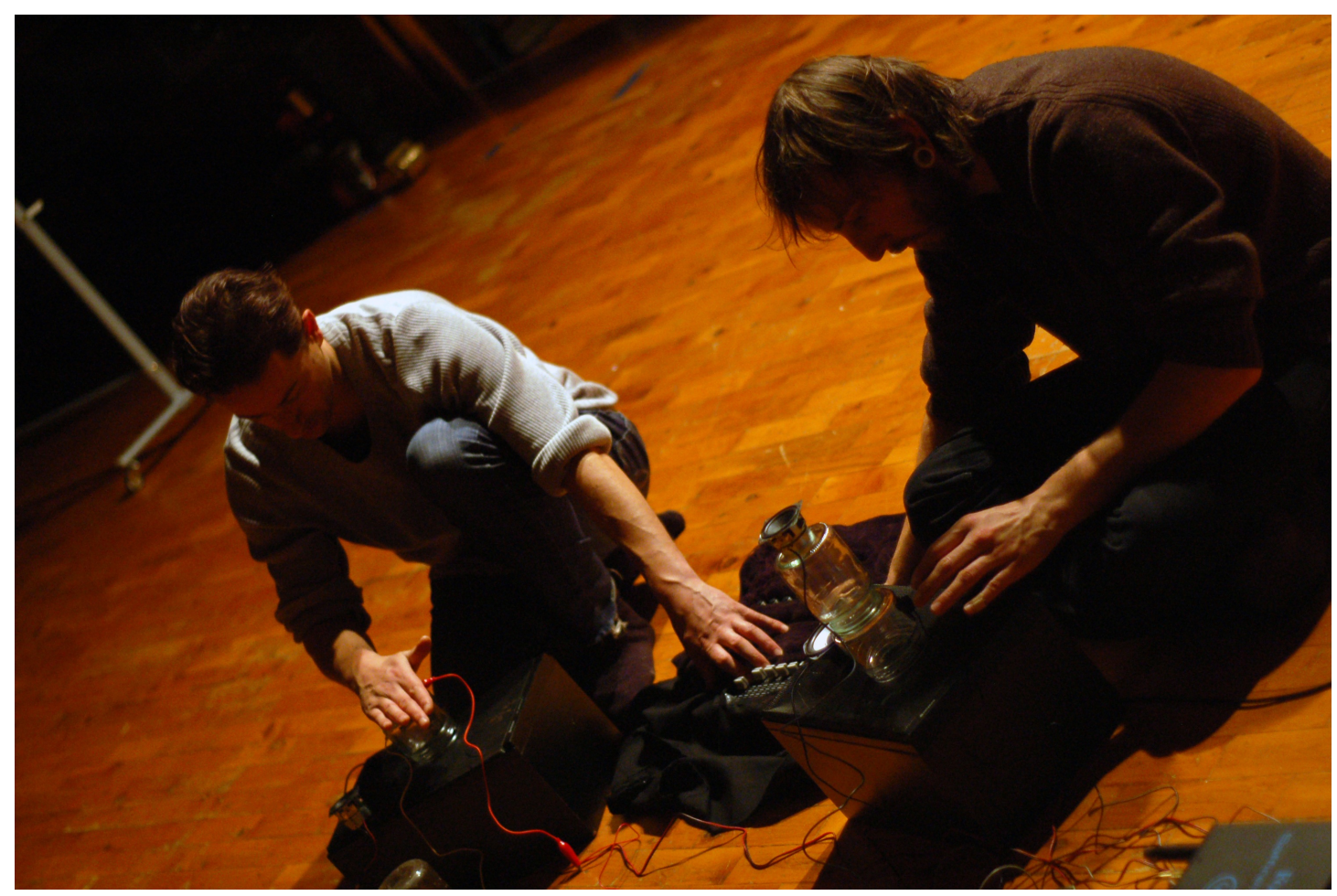

Figure 8. Glass Detail 2 


\subsection{Tender Suffocation}

Live Performance, 2012

DVD - Tender Suffocation.mp4

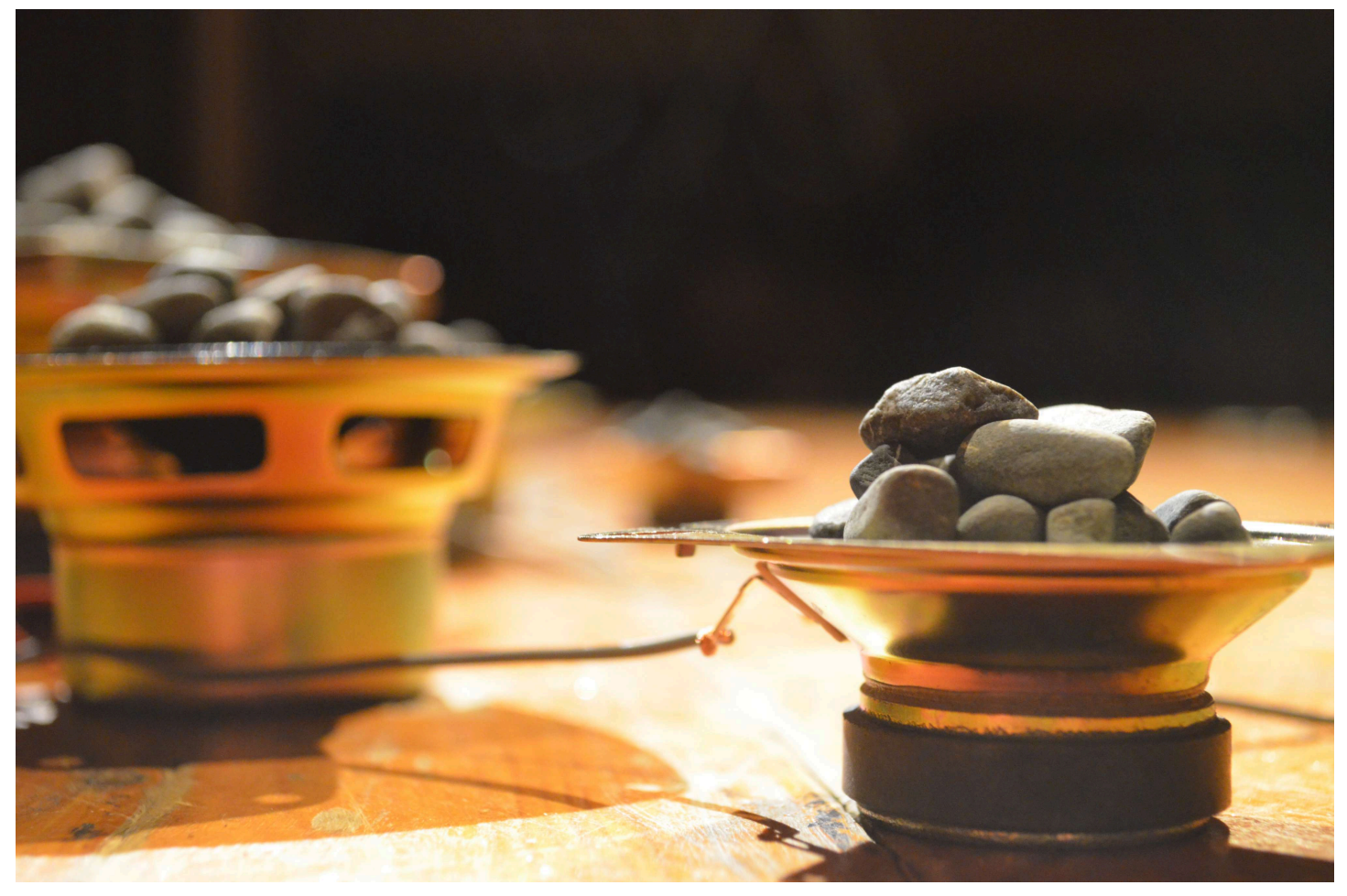

Figure 9. Tender Suffocation Detail 1

Live process piece, for five loudspeaker cones and stones 
Tender Suffocation examines the effect of objects placed upon the loudspeaker to restrict movement of the cone, and looks at how this affects the audio output of the system.

\subsubsection{Concept}

This piece is set up to mirror natural processes of erosion that occur in nature over time. The slow ritualistic movements of the performer and the inclusion of the stones taken from a local river to use as performative material combine to create an environmentally linked piece. This combined with the stark mechanics of the technology creates a conflicting aesthetic that is resolved with the natural element of the piece taking over and suppressing the man-made objects. Through this the idea of suffocation is addressed both physically and conceptually, where the natural materials are to constrict the functionality of the technology.

\subsubsection{Performance}

In the performance, five loudspeaker cones are placed on the floor and are fed precomposed sound material. This material, derived from piano and vibraphone samples, remains on a short loop that continues for the duration of the piece. As this loop plays, the performer proceeds to place small stones upon the cones. As this is done, gradually over the course of 15 minutes (the video runs for ten minutes and is edited to create a heightened awareness of the slow process), the weight of the stones starts to weigh the cone down, thus restricting its ability to fully reproduce the original sound material. As the stones accumulate upon the surface of the cone, they act not only as a weight, as mentioned, but also as a sonic filter, distorting the higher frequencies and muffling lower frequencies. As the sound loop is short and repetitive it allows the listener to assess the transformation of sound as the stones are placed upon the cones. By placing these objects on top of the loudspeakers and restricting the cones movement, three 
effects are created; as mentioned, the amplitude is decreased dramatically and the original sound becomes filtered. Lastly the sound of the stones themselves become audible as the movement of the cone causes the stones to shuffle and rub against one another, adding a layer of sound caused by the movement of the cones but external to the sound information the loudspeaker is trying to produce.

Over the course of the work there is a set process in terms of the order with which the loudspeakers are covered. The two smallest cones are chosen first. This is because they have the lowest amplitude output and the most limited frequency range. The change in the overall sound of the system after covering these cones is not hugely significant but the potential for the perceived change in the listener is. This is because there is something definite happening visually, but the audible change may be harder to perceive. As the process of the work is to slowly change the sound, changing it too much early on would be detrimental, audibly and perceptually. Next then are the middle size cones, chosen as the potential to modify the sound of these speaker's increases from the smaller cones.

Last to be covered is the largest cone, in opposition to the smaller cones, has the highest amplitude and widest frequency range of the loudspeakers present. Due to these factors, the change when the stones are placed upon it is the most substantial. The lower frequency material that has been emanating from this cone is dampened considerably, reducing the spectrum of the original piece. As the production of the higher frequency material requires less movement from the cone, these frequencies are not completely muted, but are left distorted and altered, combined with the sounds of the stones themselves.

When comparing the audio from the beginning of the piece and the end of the piece, there are definite changes to both the spectrum and amplitude of the audio. This is not 
so easily perceived when viewing the work in a linear fashion as the changes happen gradually. The visual component of the work, i.e. the slow placing of stones upon the loudspeakers, helps in this perception as it implies that the visual change corresponds to an audible change, even if this change is only a minor one.

\subsubsection{Referential Material}

As possibilities for the conceptual element of the piece, suggestions for using the voice as sound material were given as this would relate to the body and the idea of suffocation more explicitly. Although this may have been conceptually interesting, also taking into account the recognisability of the changes to take place, I felt the voice might draw the listener away from the transformations themselves and lead them towards a struggle for possible semantic meaning. The outcome of using the voice, or indeed another strongly referential sound source, is evident in my installation Deaf of the Author (2011). Here the voice within the piece Mirage (2007) by Kaija Saariaho was used to gain the effect of recognisability and allow for easier examination of the sound through the loudspeaker. This proved to lead to causal and semantic listening, rather than the reduced listening ${ }^{8}$ that $\mathrm{I}$ had intended.

\footnotetext{
${ }^{8}$ Chion, 1994
} 


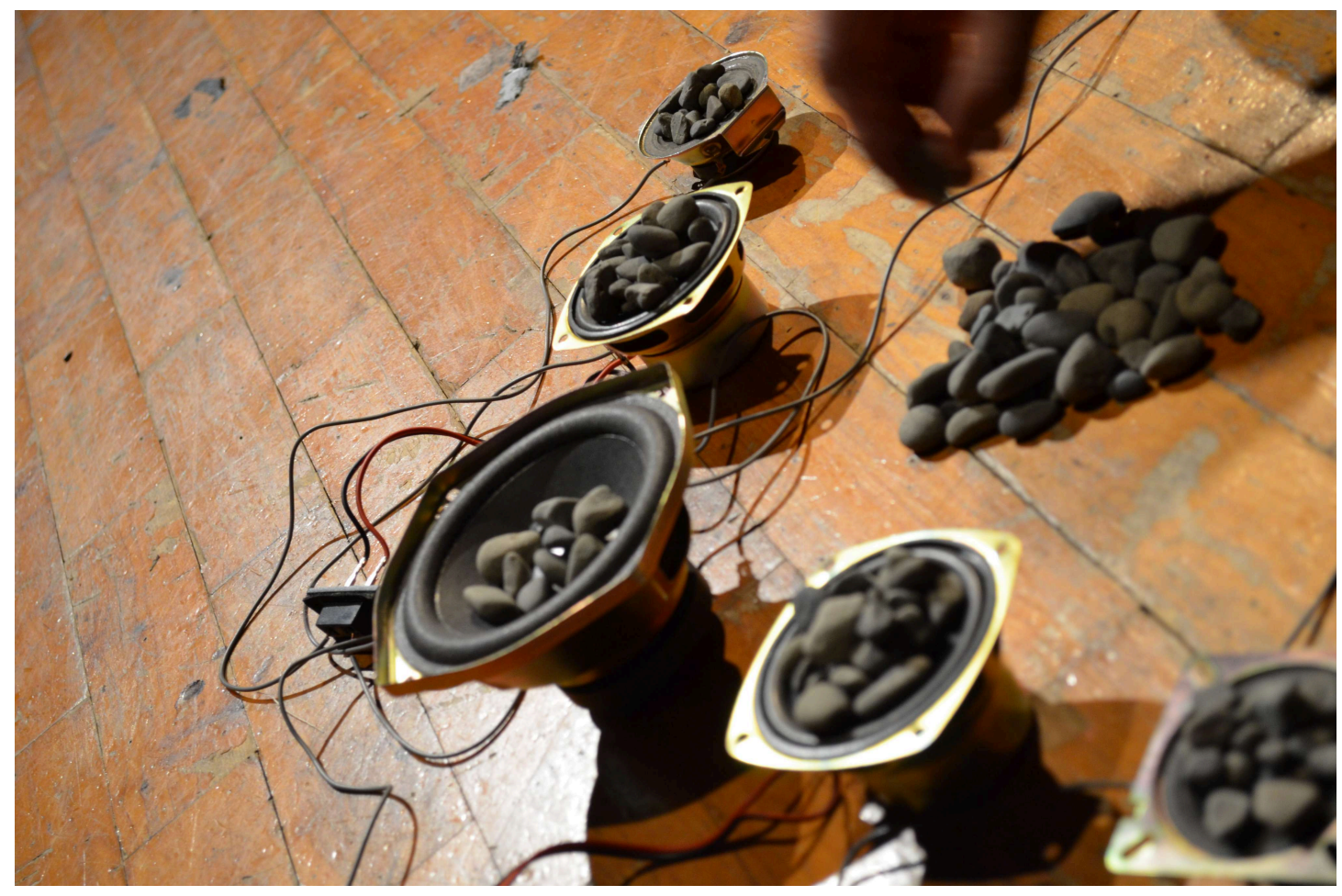

Figure 10. Tender Suffocation Detail 2 
5. Sound Installations 


\subsection{Deaf of the Author}

Sound Installation, 2011

DVD - Deaf of the Author.mp4

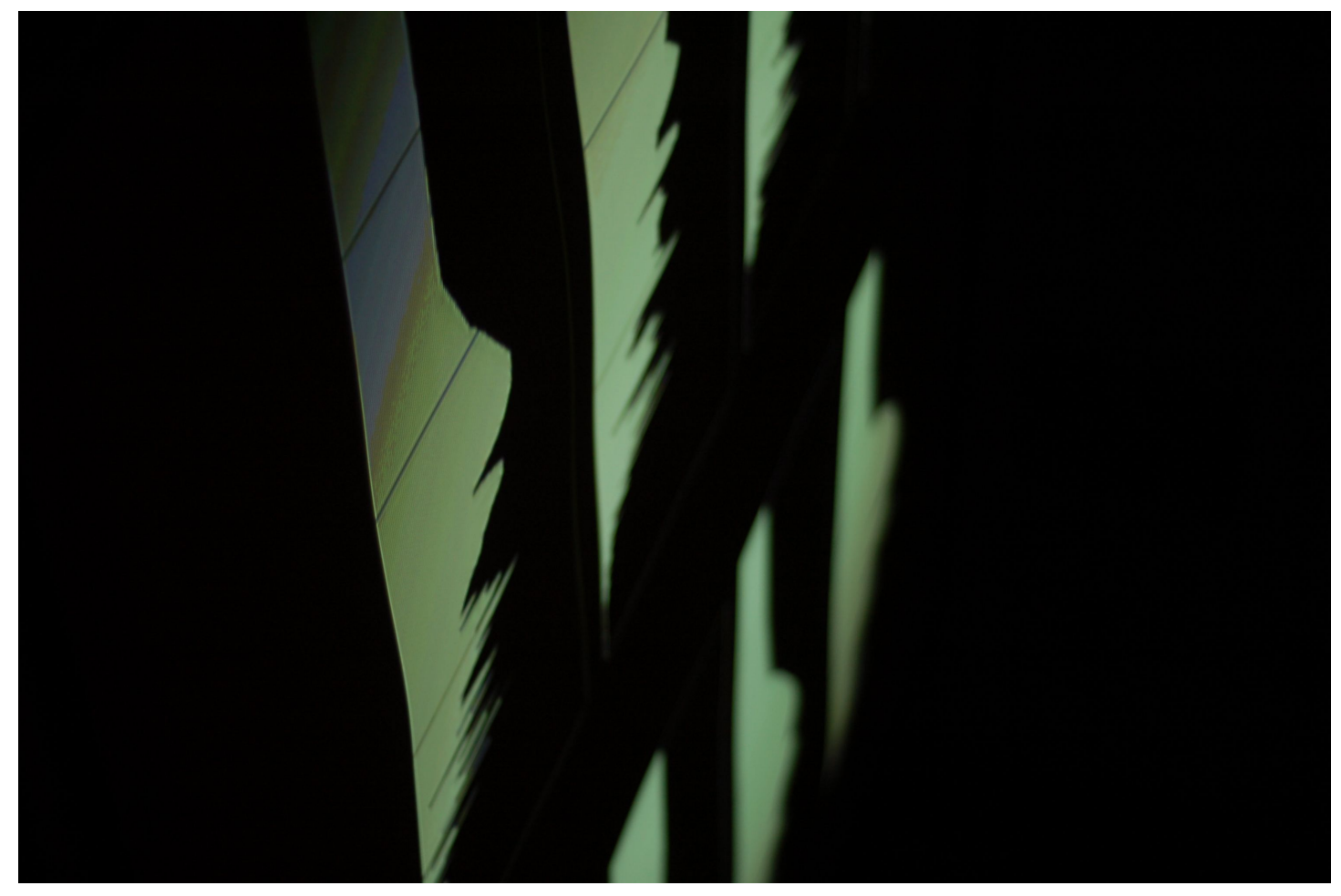

Figure 11. Deaf of the Author Detail 1

This Sound Installation was held in the Adam Concert Room on Friday $7^{\text {th }}$ October 2011

Programme Note:

'We listeners have become arrangers. Adorno understood this, writing in 1938, "The sonorous modifications that every orchestra undergoes when it resounds in a room through loudspeakers are themselves a kind of arrangement."' (Szendy, 2008) 


\subsubsection{Concept}

Drawing on Roland Barthes' idea of the death of the author ${ }^{9}$ as conceptual inspiration, this installation plays on the ability of the loudspeaker to dispel with the composers intentions concerning the precisely determined intrinsic elements of the mix of a piece of music. Drastically shaping a piece of music according to the physical parameters of the object, differing loudspeakers create flux in frequency, spectrum and the accentuation of materials within any specific work.

Through the juxtaposition of visual and audible information, concerning the physical parameters of the loudspeaker, listeners will be encouraged to decipher and reconcile what information they are given with the audible information they perceive.

Minimal information is given to the audience member about the concept, aim or potential outcomes of the piece. This is to help them engage in their own personal interpretation with minimal intervention from myself, the composer. In keeping with my original concept, my intention for the work no longer matters as the evaluation of the audience member becomes conclusive.

\subsubsection{Method}

A fixed media piece was created (detailed below). This piece was then played and recorded through sixteen specially selected loudspeakers. These loudspeakers were chosen for their range in size, condition and materials, to enhance the range of individuality the objects could project upon the fixed media piece. The loudspeakers were each measured, with both the encasing dimensions and the dimensions of the cones taken. Along with the dimensions, the materials, frequency responses and number of cones were recorded. This information was then correlated and printed out on a

${ }^{9}$ Barthes, 1967 
document along with the resulting waveform of the fixed media piece, transformed by that loudspeaker.

For the installation the fixed media piece runs twice through to comprise a single section. The first run-through plays loudspeakers 1-8 and the second run-through plays loudspeakers 9-16. At the end of this second instance, the whole piece loops again continuously.

For the video component of the installation, all sixteen resultant pieces, 1-8 first then 916, were fed into a spectrum analyser in Max/MSP. These were setup in a way that mapped out the physical positions of the speakers that were to sit on the floor within the installation space. While the spectrum video played through, this was recorded then digitally processed to render pulsing and strobe effects.

Within the installation space, eight identical loudspeakers (Genelec, 1038B) were setup. This was to allow the listener unbiased perception towards the sound of any particular speaker via direct visual clues. Having the original speakers in the space would allow the listener to gain an idea of what the piece may sound like, thus listening more to the piece than to the sound of the particular speaker. Through playing the fixed media through identical loudspeakers, what they are essentially listening to is the sound of another loudspeaker. Further to this, the Genelecs then produce their own version of each of the audio renderings, producing another level of authorship upon the fixed media.

The size and quality of the installation speakers is directly related to the perceptions of the resulting sound. The large size of the speakers implies a full range frequency response. This tells the listener that any sound produced by these speakers is not constrained by an insufficient frequency limitation, and is most likely to be reproduced accurately, or, as the composer may have intended. 


\subsubsection{Sound Material}

The sound material used to create the fixed media piece for the installation consisted of two pre-composed works; La Roue Ferris (1971) by Bernard Parmegiani and Mirage (2007) by Kaija Saariaho. These works were chosen for two reasons; firstly the Parmegiani piece was chosen for its extremely wide frequency range. This was to ensure maximum activation of each loudspeaker used, thus bringing out any specific characteristics and limitations of the said objects. The Saariaho work was chosen for its use of the voice and string instruments. These were to serve as an everyday reference for the listener, who would then be able to compare the sound of the voice in each speaker. The voice becomes important in the work as the listener may also be drawn into gaining semantic meaning, thus engaging in closer listening.

The fixed media piece was created by layering these two works over each other. Minimal editing of the original pieces was done to make sure the pieces were both the same length. Volume was also automated to bring out particular features of both works at certain points, for example the voice in Mirage was accentuated at points to contrast with the synthesized material in La Roue Ferris. Conceptually, the authorship placed upon these works by myself (to create a third work) through editing, mirrors my original standpoint about the nature of the original author. As mentioned in the programme note for the installation: [Pieces copied, converted, cut, and re-recorded without permission from the authors]. Whilst this is an important factor of the installation, this remains a conceptual component. What is the intended focus, is the differentiation between the renditions of this fixed media piece, across the numerous loudspeakers. 


\subsubsection{Information Printout}

This information printout ${ }^{10}$ contains information about each individual loudspeaker. This includes, frequency response, casing material and dimensions of the body and cone/s of the loudspeaker. Along with this is a waveform of the fixed media piece, as rendered by the particular loudspeaker. The information is provided as a means to inform the audience about the nature of the loudspeaker used to re-record the fixed media piece. The intention here is to create an overbearing sense of information that is not intuitively helpful for gaining an understanding of the audible output. Apart from the waveform, most of this information is not taken into account when listening over loudspeakers on an everyday basis and may be confusing for the audience member.

\subsubsection{Video Component}

The video component ${ }^{11}$ of the installation functions in two ways:

Firstly, the video component allows for direct comparison, in real time alongside the audio, of the frequency spectrum readouts for each speaker. This allows comparison and the chance to see differences between each loudspeakers response to the fixed media input. The overall waveforms are reproduced and placed on top of each speaker, but because of only showing subtle differences it is hard to compare multiple waveforms at once.

Secondly, the flashing and pulsing of the video is intended to be disorientating. This is to be complementary to the information on the printout. Alongside this, the strobe effect of the video makes the printouts hard to read and creates an effect where only flashes of the printout can be seen at a time, creating an overall fragmented effect.

\footnotetext{
${ }^{10}$ See Deaf Information Printout.pdf in 'Supplementary Material' in Portfolio DVD.

${ }^{11}$ See Deaf Video Component.mov in 'Supplementary Material' in Portfolio DVD
} 
To work with the video, the level of lighting also needed to be considered. The dimmed lights allow the strobe-like effect of the video component to be intense and startling. This provides brief periods of illumination for the listener to decipher the written information without this information becoming overbearing upon the aural information in the work.

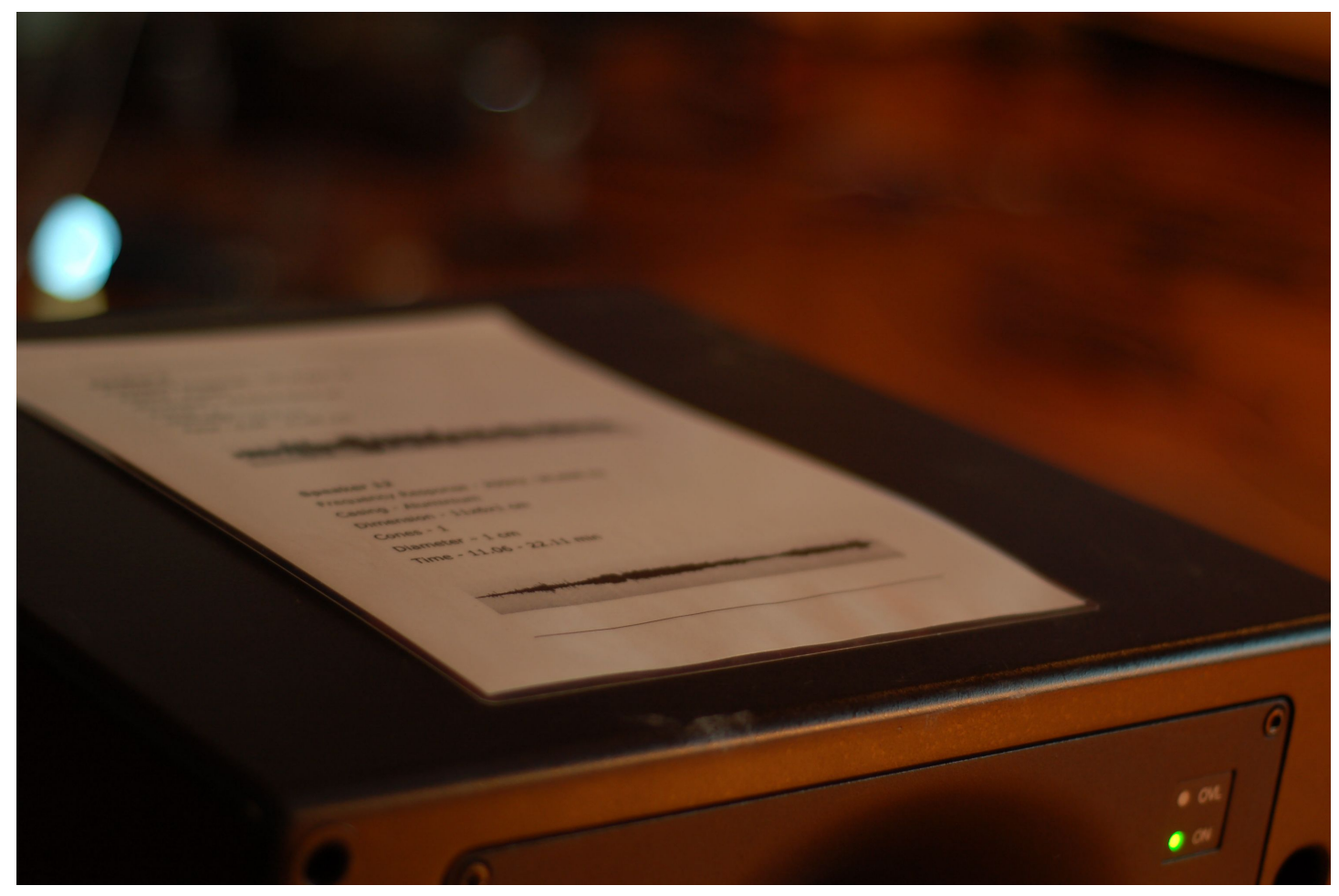

Figure 12. Deaf of the Author Detail 2 


\subsection{Jukebox}

Sound Installation, 2011

DVD - Jukebox.mov

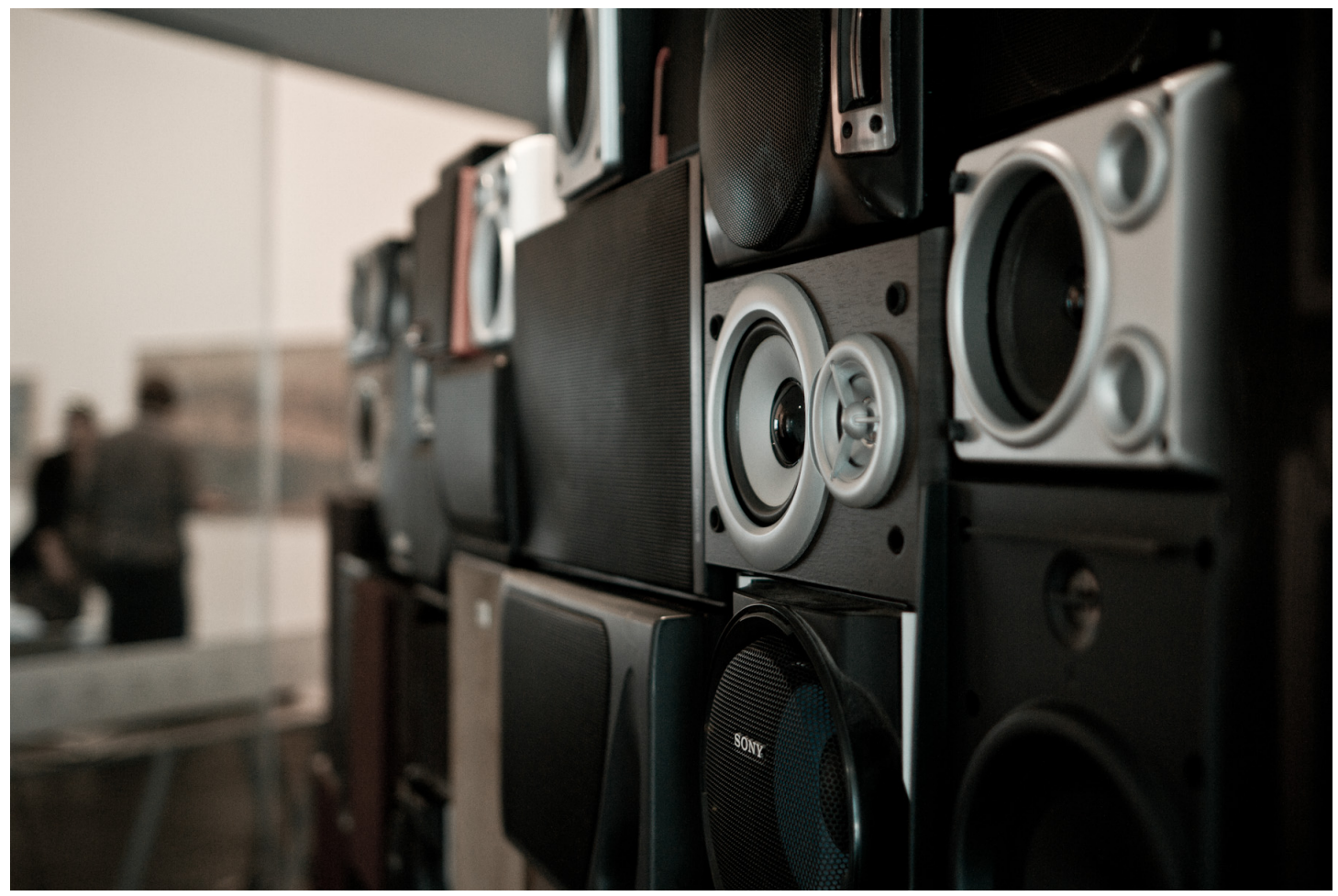

Figure 13. Jukebox Detail 1

Jukebox was held in the space between the sliding doors of the Adam Art Gallery ${ }^{12}$ from November 14th to December $19^{\text {th }} 2011$.

$12<$ http://www.adamartgallery.org.nz/> (28 May 2012) 


\subsubsection{Programme Note}

'Jukebox is an attempt to force the loudspeaker back into consciousness and draw attention to it as an entity as important to music reception as any other technology that has informed the creation of music. The music played through the installation is an assemblage of material collated from participants at the opening night event. The various sounds filtered through the installation serve to highlight the loudspeaker as an instrument integral to how we perceive sound and music. This sound installation will be presented between the sliding doors of the Adam Art Gallery until the end of the year.'

\subsubsection{Overview}

Jukebox is a speaker sculpture, consisting of 46 loudspeakers stacked in a wall-like formation. These loudspeakers are setup in series connected to a single stereo amplifier so they are all able to receive the same audio information at the same time. The speakers used are of varying shape, size, material, quality, colour and age. My aim in this installation was to bring attention to the object, the loudspeaker, and the effect it has on intrinsic qualities of music auditioned over it. ${ }^{13}$ Allowing the listener to audition music over the various loudspeakers at the same time encourages this, as presence of the object itself demands to be addressed and comprehended before the music. The visual and audible information combine to inform a particular perception of the music played.

The concept of the loudspeaker transforming the musical object has also been addressed in my installation Deaf of the Author (2011). The concept in Deaf of the Author was contextualised differently to Jukebox and framed in a way that an audience member would have to decipher audible and visual information provided in order to reach an understanding of my conceptual intentions. Jukebox by contrast provides a more accessible route towards conceptual understanding of the piece.

\footnotetext{
${ }^{13}$ Discussed in full in Chapter Four of Exegesis, Transformation of the Musical Object
} 
As each loudspeaker has its own particular sonic characteristics, attributed to its particular anatomy, it follows that any sound information passed through this loudspeaker is shaped by these particular characteristics. By placing multiple loudspeakers side by side and in direct proximity of one another, the chance to audition them and gain instant aural feedback is gained. Playing on the perception of sound informed by the visual, in this case the various elements of the loudspeakers mentioned above, Jukebox encourages the listener not only to hear, but to see the difference in sound. This may seem counter-intuitive at the time, but this does allow the audience member to become aware of the physical attributes of the loudspeaker that inform the music, thus perceiving sound differently according to visual stimulus.

A further means to heighten this, was to convert all audio in Jukebox to mono, making the stereo field negligible, but allowing more focus upon the elements of the mix. This was done as the setup of the speakers and the size of the space was not conducive to replicating the stereo field accurately. Not all loudspeakers had a stereo pair and not all that did were placed directly opposite one another. This creates a level of authorial transformation of the materials used in the work.

\subsubsection{Audience Participation}

As mentioned in the programme note, participants of the opening night event at the gallery were invited to bring along music of their choice. This was to be gathered and used as the musical material for the duration of the exhibition. Audience participation was integral to the work for three reasons:

Firstly, I wished to have no personal input concerning the audio played through the sculpture. As I wished to have a wide range of materials and a chance element concerning the audio, I felt that to choose audio myself would lead to an amount of aesthetic choice that would colour the work too distinctly. Allowing the audience to 
choose allowed for the chance element of choice to be realised and a range of materials to come forward, some unfamiliar to me, to be played through the work.

Secondly, for the perception of change in the piece to occur in the listener, they would have to choose something familiar to themselves. A familiar piece of music given multiple listens allows a sense of stasis about intrinsic elements of the piece, thus any changes to the said piece may be brought to the forefront of the listeners perception (This being said, I was working on the assumption that the participants would bring in music familiar to them, whereas this may not always be the case. The third point below takes over in the cases where the music brought in is not so familiar to the listener).

Thirdly, this level of participation in the work leads to a sense of personal investment that may lead the participant to engage more fully and actively in the work and the effect it is trying to achieve. I found this to be the case as audience members were more likely to stay for the entire duration of his/ her musical choice, paying more attention to their work than any other. This ties in to the level of approachability I was trying to evoke for this work; the level of personal contribution not only affects the direct contributors, but also provides vicarious means for other audience members to engage the work by way of relating to these particular musical choices.

\subsubsection{Audience Engagement and Response}

Although simple in concept and execution, Jukebox brings to light some elements of the loudspeaker that are not usually at the forefront of our attention and perception, these being ???. Complementary to this, the installation draws attention to surrounding elements of music production and reception. Elements of social archaeology and personal preference are brought into the work through audience participation and the element of chance associated with this. 
As participants are invited to bring along musical material of their choice, the aural material of the piece is unknown before the opening event. This creates an element of audience specificity leading to a possibility of multiple instances of the installation with varying content, whilst retaining the underlying concept. An important consequence of this chance element is the affect of the music chosen. Individual participants may be familiar with their own choices of music, but have yet to hear the choices of the other contributors. This brings into the installation elements of nostalgia, emotional resonance and musical curiosity where an audience member may be drawn into a particular piece of music because of personal interest. Concurrently, because of these reasons the listener may be drawn into the music's extrinsic levels that may not allow them to engage fully with the sculpture and the aural repercussions of the multiple loudspeakers.

In contrast to this, a deeper connection with the piece of music may lead to a greater engagement with the music and sculpture as a whole. Although a key concept of the installation is familiarity of the musical object heard, it does not rely solely on this as the installation serves to highlight any piece of music played.

One aspect of the sculpture I did not account for was the nostalgia factor of the loudspeaker itself. Various comments from audience members were aimed towards the similarity of some of the speakers to the ones they used to have, or the ones their parents had. The sculpture became a point of reference for reminiscing, not about the music itself, but about previous houses or pieces of furniture. ${ }^{14}$

One comment from an audience member was directed at recognising the difference in sonic quality of music from different eras. Whilst this may be an accepted and obvious fact, this shows the frame of mind listeners were drawn into whilst viewing the work.

\footnotetext{
${ }^{14}$ Discussed in full in Chapter Six of Exegesis, Visual
} 


\subsubsection{Sculpture}

Spatially, Jukebox becomes quite sonically dynamic, as movement across the sculpture is created according to the physical frequency response of the individual speakers. Depending on the specific mix of each track, it may move vertically between the larger and smaller speakers. This is caused by the physical structure of each loudspeaker and the particular EQ of the mix. Larger speakers are activated when there is more bass present. This gives the impression of the sound moving vertically up to the smaller speakers when there is more treble in the mix. Caused by the directionality of the speakers, moving ones head across the sculpture gives the effect of moving through the different sound fields of the speakers.

What Jukebox does allow is a transition into the 'thingness' of the object. Through the movement of sound across the installation, as just outlined, and the slightly unexpected effect of finding it hard to localise the sound emanating from the sculpture due to the lack of stereo pair reference and the varying volumes between speakers (also the particular acoustic of the space), a sense of detachment of the sound and the object producing it is invoked. This results in a separation of the two objects, the loudspeaker and the music, which come to be perceived as independent from each other. 


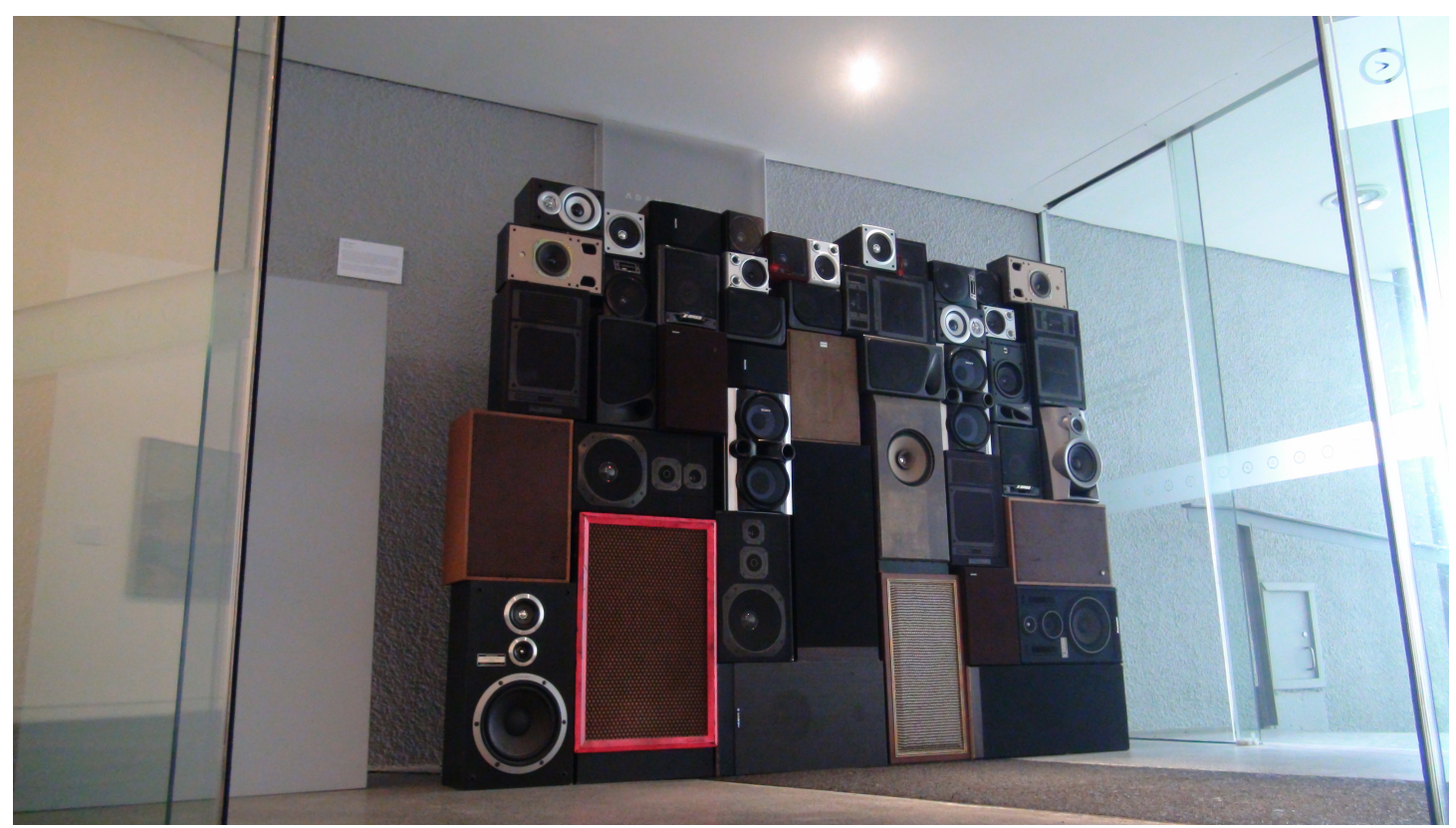

Figure 14. Jukebox Detail 2 


\section{Portfolio Credits}

All performance, photography, video, sound recording and editing done by Jason Wright unless otherwise stated.

\section{Allele:}

Sound Recording - Jason Post

\section{Blue:}

Performer - Bridget Johnson

Photography - Jim Murphy

Video - Jim Murphy

Sound Recording - Jim Murphy

Deaf of the Author:

Photography - Jim Murphy

\section{Glass:}

Performer - seablack

Video - Jim Murphy

\section{Jukebox:}

Photography - Boofa and Jason Wright

\section{Tender Suffocation:}

Video - Jim Murphy

Photography - Jim Murphy and Jason Wright 
Sound Recording - Bridget Johnson 


\section{Bibliography}

Adam Art Gallery: <http://www.adamartgallery.org.nz/>. (28 May 2012)

Barthes, Roland (1977). “The Death of the Author", in Image, Music, Text, trans

Stephen Heath, New York, N.Y. : Noonday Press, 1988.

Chion, Michel (1994). Audio-Vision: Sound on Screen, trans. Claudia Gorbman,

Columbia University Press, New York.

Helmholtz Resonance. <http://www.phys.unsw.edu.au/jw/Helmholtz.html> (28 May 2012).

New Oxford American Dictionary (2005). 2nd edition, Oxford University Press, Inc.

Szendy, Peter (2008). Listen: a history of our ears, New York : Fordham University Press, 2008.

\subsection{Works Cited}

Parmegiani, Bernard (1971). La Roue Ferris, Disc 3, Institut national de l'audiovisuel (France). Collection GRM. Paris : INA, 2008.

Saariaho, Kaija (2007). "Mirage", in Saariaho - Notes On Light, Orion \& Mirage, Ondine, catalogue no. ODE11302, 2008. 